\title{
OVERGROUPS OF PRIMITIVE GROUPS
}

\author{
MICHAEL ASCHBACHER \\ (Received 1 September 2007; accepted 6 June 2008) \\ Communicated by E. A. O’Brien
}

\begin{abstract}
We give a qualitative description of the set $\mathcal{O}_{G}(H)$ of overgroups in $G$ of primitive subgroups $H$ of finite alternating and symmetric groups $G$, and particularly of the maximal overgroups. We then show that certain weak restrictions on the lattice $\mathcal{O}_{G}(H)$ impose strong restrictions on $H$ and its overgroup lattice.
\end{abstract}

2000 Mathematics subject classification: primary 20B15; secondary 20B35, 20D30.

Keywords and phrases: permutation group, lattice.

\section{Introduction}

Assume that $\Omega$ is a finite set, $S=\operatorname{Sym}(\Omega)$ is the symmetric group on $\Omega, G$ is $S$ or the alternating group $A$ on $\Omega$, and $H$ is a subgroup of $G$ acting primitively on $\Omega$. In this paper we study the set $\mathcal{O}_{G}(H)$ of overgroups of $H$ in $G$. In particular, we describe (in some sense) the maximal overgroups of $H$. Then we use our description to establish results about the lattice $\mathcal{O}_{G}(H)$ when $H$ is the intersection of a pair of maximal subgroups of $G$.

In [P], Cheryl Praeger also discusses the overgroups of primitive subgroups of alternating and symmetric groups, but her organization of information does not immediately lend itself to the applications we have in mind. Thus we have produced our own treatment, which we believe has some advantages over that in [P].

In [P], inclusions among primitive subgroups of $S$ are described in terms of the notion of a 'blow-up'. In this paper, the maximal subgroups of $S$ are described (whenever possible) as stabilizers of suitable structures on $\Omega$. Inclusions among primitive subgroups are described in terms of the embedding of generalized Fitting subgroups, and relations among the structures preserved by the subgroups, particularly the product structures on $\Omega$. In a sequel to this work, we introduce a partial order on product structures; together with inclusions of generalized Fitting subgroups, this

This work was partially supported by grant no. NSF-0504852.

(C) 2009 Australian Mathematical Publishing Association Inc. 1446-7887/2009 \$16.00 
partial order gives a qualitative description of large portions of the poset of primitive subgroups of $S$.

We now give some details of the work. See [A2] for the notation and terminology involving finite groups used in the paper.

We begin with a brief discussion of the relevant structures on $\Omega$. Informally, an affine structure on $\Omega$ is an identification of $\Omega$ with the points of an affine space; see Definition 1.2 for a precise definition. The notion of a diagonal structure is less natural; see Definition 1.9 for a definition.

Informally, a regular $(m, k)$-product structure on $\Omega$ is an identification of $\Omega$ with the set product of $k$ copies of a set of order $m$, but often it is better to view the product structure as a certain kind of chamber system on $\Omega$. (As defined by Tits in [T], a chamber system on $\Omega$ is a collection of equivalence relations on $\Omega$.) The referee has pointed out that the latter point of view is also adopted in [BPS1], where product structures are called 'Cartesian decompositions', and that Baddeley and co-workers [BP, BPS1, BPS2, BPS3] prove various results about Cartesian decompositions, and about quasiprimitive and innately transitive subgroups of $S$ preserving Cartesian decompositions. (Quasiprimitive and innately transitive groups are classes of permutation groups more general than the class of primitive groups.) The referee also points out that it should be possible to give a shorter proof of our propositions below, using $[\mathrm{P}]$ and these references. However, we feel that a description (in terms of natural structures on $\Omega$ ) of the poset of primitive subgroups of the symmetric groups is sufficiently fundamental to warrant a self-contained treatment that avoids an invocation of the notion of a blow-up and appeals to the broader literature on innately transitive groups.

Next we recall some facts about primitive subgroups $H$ of $S$. These facts are stated in terms of the generalized Fitting subgroup $F^{*}(H)$ of $H$, so we recall that $F^{*}(H)$ is a certain characteristic subgroup of $H$ which controls the structure of $H$. The notion of the generalized Fitting subgroup plays a crucial role in finite group theory; see [A2, Section 31] for a discussion of this topic. Set $D=F^{*}(H)$. The possibilities for the pair $H, D$ and their action on $\Omega$ were determined in [AS], and are listed in Lemma 2.2. By now they are well known. In the language of Definition 2.3, there are five types of primitive groups $H$ : affine, doubled, semisimple, diagonal, and complemented groups. When $H$ is affine, $D$ is the direct product of subgroups of order $p$ for some prime $p$, and is regular on $\Omega$. In the remaining cases, $D$ is the direct product of isomorphic nonabelian simple groups, and the type of $H$ is determined by the embedding of a point stabilizer $D_{\omega}, \omega \in \Omega$, in $D$.

There are some interesting special cases: the almost simple groups, the strongly diagonal groups (see Definition 2.3), the product decomposable semisimple groups (see Definition 5.10), and the octal semisimple groups (see Definition 4.2). An almost simple group is product indecomposable if it preserves no nontrivial product structure. The general semisimple group $H$ is product decomposable if the obvious product structure $\mathcal{F}(H)$ preserved by $H$, when 'composed' with the rank-two product structure preserved by a component of $H$ (using the composition defined in Definition 1.11), 
produces a larger $H$-invariant product structure. An octal semisimple group $H$ exists only when $\Omega$ has order a power of 8 , and $D$ preserves an affine structure.

There are four kinds of primitive groups maximal in $G$, as listed in Lemma 2.5.

(1) The affine maximals, which are the stabilizers of affine structures on $\Omega$.

(2) The semisimple maximals, which are the stabilizers of regular product structures on $\Omega$.

(3) The strongly diagonal maximals, which are the stabilizers of diagonal structures.

(4) The almost simple maximals.

See Section 1 for a discussion of the various structures.

The statements of our various propositions and theorems involve some specialized notation. We begin our discussion of that notation with a few words which hopefully will give the reader some flavor of the objects described by the notation. Then we direct the reader to the places in the paper where precise definitions appear. First, $\mathcal{F}(H)$ is the set of $H$-invariant product structures on $\Omega$, while $\mathcal{F}(H)$ is a certain natural member of $\mathcal{F}(H)$. It turns out that $\mathcal{F}(H)$ is the greatest member of $\mathcal{F}(H)$ in a certain partial order, unless $H$ is semisimple and decomposable, where $\mathcal{F}^{2}(H)$ is the greatest member. One can parameterize $\mathcal{F}(H)$ in terms of a certain collection of subgroups $K$ of $H$ via bijections $K \mapsto \mathcal{F}(H, K)$ or $K \mapsto \mathcal{F}^{2}(H, K)$, with $H$ semisimple and product decomposable in the latter case. When $H$ is diagonal, $\Sigma(H)$ is a certain partition of the components of $H$. Finally, when $H$ is affine, $\mathcal{D}(H)$ is the set of nontrivial $H$-invariant direct sum decompositions of $D$.

A precise definition of the notation $\mathcal{F}(\mathcal{D})$ and $\Sigma(H)$ appears in Example 1.6 and Lemma 2.2, respectively. The notation $\mathcal{D}(H), \mathcal{F}(H), \mathcal{F}(H)$, and $\mathcal{F}(H, K)$ is defined in Notation 2.6. Finally, the definition of $\mathcal{F}^{2}(H)$ and $\mathcal{F}^{2}(H, K)$ appears in Lemma 5.11(6).

We wish to investigate the set

$$
\mathcal{O}_{G}(H)^{\prime}=\left\{M \in \mathcal{O}_{G}(H) \mid F^{*}(G) \not \leq M\right\}
$$

of 'proper' overgroups of $H$ in $G$ and, in particular, the set $\mathcal{M}(H)$ of maximal members of $\mathcal{O}_{G}(H)^{\prime}$. As discussed above, there are five types of primitive groups; however, for the purposes of our propositions, it is convenient to regard the almost simple primitive groups as forming a sixth type. Roughly speaking, if $H$ is of type $T_{H}$ and $M \in \mathcal{O}_{G}(H)^{\prime}$ is of type $T_{M}$, then there are fairly strong restrictions on the pair $\left(T_{H}, T_{M}\right)$, and often $F^{*}(M)$ is forced to be $F^{*}(H)$. Indeed, if $M$ is not semisimple then the embedding of $H$ in $M$ is essentially completely controlled by the pair $T_{H}$, $T_{M}$, and the embedding of $F^{*}(H)$ in $M$, so the situation is quite satisfactory. On the other hand, when $M$ is semisimple, things are more complicated, but at least $H \leq M$, so $\mathcal{F}(M) \subseteq \mathcal{F}(H)$, and the maximal semisimple overgroups of $H$ are in one-to-one correspondence with $\mathcal{F}(H)$, which we describe in terms of parameterizations that appear in the various propositions.

We organize this information into eleven propositions of two kinds. The first kind of result fixes the type of a primitive subgroup $M$, and describes the possible 
primitive subgroups of $M$, according to type and other restrictions. The second kind of result fixes the type of $H$, and describes the members of $\mathcal{O}_{G}(H)^{\prime}$, again according to type and other restrictions. There are so many inclusions of primitive groups in semisimple groups, that it makes little sense to state a proposition in the case where $M$ is semisimple. We continue to write $D$ for $F^{*}(H)$.

Proposition 1. Assume that $M \in \mathcal{O}_{G}(H)^{\prime}$ is almost simple. Then either

(1) $H$ is almost simple; or

(2) $|\Omega|$ is a prime and $H$ is affine.

Proposition 1 is proved as part of the proof of Lemma 4.4.

Proposition 2. Assume that $M \in \mathcal{O}_{G}(H)^{\prime}$ and that $H$ is almost simple. Then one of the following statements holds:

(1) $M$ is almost simple;

(2) $|\Omega|=8, H \cong L_{3}(2)$ is octal, $M$ is the stabilizer of one of the two $H$-invariant affine structures on $\Omega$, and the two structures are conjugate under $N_{S}(H)$;

(3) $H$ is product decomposable, $M$ is semisimple, and $M$ is contained in the stabilizer $N_{G}\left(\mathcal{F}^{2}(H)\right)$ of $\mathcal{F}^{2}(H)$.

Proposition 2 is a corollary to Proposition 5. The statement in part (2) of the proposition that $H$ stabilizes two affine structures follows from Lemma 7.1(5).

Proposition 3. Assume that $M \in \mathcal{O}_{G}(H)^{\prime}$ is affine and let $X=F^{*}(M)$. Then either

(1) $H$ is affine and $F^{*}(M)=F^{*}(H)$; or

(2) $\Omega=8^{r}, H$ is octal semisimple with components $\left\{L_{1}, \ldots, L_{r}\right\}, H X$ is affine with $\mathcal{D}=\left\{\left[X, L_{i}\right] \mid 1 \leq i \leq r\right\} \in \mathcal{D}(H X)$, and $\mathcal{F}(H)=\mathcal{F}(\mathcal{D})$.

Proposition 3 follows from Lemma 4.3.

Proposition 4. Assume that $M \in \mathcal{O}_{G}(H)^{\prime}$ and that $H$ is affine. Then one of the following statements holds:

(1) $M$ is affine and $F^{*}(M)=F^{*}(H)$;

(2) $M$ is semisimple, $H$ is imprimitive on $D$, and there exists $\mathcal{D} \in \mathcal{D}(H)$ such that $\mathcal{F}(\mathcal{D})=\mathcal{F}(M)$

(3) $|\Omega|$ is prime and $M$ is almost simple.

Proposition 4 follows from Lemma 4.1. If $|\Omega|$ is prime and (1) fails, then $M$ is almost simple from Lemma 2.2.

The next three propositions are proved in Section 7.

Proposition 5. Assume that $H$ is semisimple and pick a component $L$ of $H$. Let $M \in \mathcal{O}_{G}(H)^{\prime}$.

(1) If $H$ is product indecomposable and not octal then $M$ is semisimple, $L$ is contained in a component of $M, H$ is transitive on the components of $M$, and the map $K \mapsto \mathcal{F}(H, K)$ is a bijection of $\mathcal{O}_{H}\left(N_{H}(L)\right)^{\prime}=\mathcal{O}_{H}\left(N_{H}(L)\right)-\{H\}$ with $\mathcal{F}(H)$. 
(2) Suppose that $H$ is octal with $k$ components. Then $M$ is affine or semisimple. Furthermore, the affine structures stabilized by $H$ are conjugate under $N_{S}(H)$. If $M$ is semisimple then $L$ is contained in a component of $M, H$ is transitive on the components of $M$, and the map $K \mapsto \mathcal{F}(H, K)$ is a bijection of $\mathcal{O}_{H}\left(N_{H}(L)\right)^{\prime}$ with $\mathcal{F}(H)$.

(3) Assume that $H$ is product decomposable. Then $M$ is semisimple and the map $K \mapsto \mathcal{F}^{2}(H, K)$ is a bijection of $\mathcal{O}_{H}\left(N_{H}\left(A_{1}\right)\right)^{\prime}$ with $\mathcal{F}(H)$, where $A_{1} \in$ $\mathcal{O}_{L}\left(L_{\omega}\right)$ is described in Definition 5.10.

Proposition 6. Assume that $M \in \mathcal{O}_{G}(H)^{\prime}$ is diagonal and let $X=F^{*}(M)$ and $k$ be the number of components of $M$. Then one of the following statements holds:

(1) $H$ is diagonal, $F^{*}(M)=F^{*}(H)$, and $\Sigma(M)=\Sigma(H)$;

(2) $H$ is doubled, $F^{*}(M)=F^{*}(H)$, and $k=2|\Sigma(M)|$;

(3) $H$ is complemented with $|\Sigma(M)|$ components, $F^{*}(M)=D C_{G}(D)$, and $k=2|\Sigma(M)|$.

Proposition 7. Assume that $H$ is diagonal and pick $\sigma \in \Sigma(H)$. Let $M \in \mathcal{O}_{G}(H)^{\prime}$. Then either

(1) $M$ is diagonal, $F^{*}(M)=F^{*}(H)$, and $\Sigma(M)=\Sigma(H)$; or

(2) $M$ is semisimple, each component of $H$ is contained in a component of $M, H$ is transitive on the components of $M$, and the map $K \mapsto \mathcal{F}(H, K)$ is a bijection of $\mathcal{O}_{H}\left(N_{H}(\sigma)\right)^{\prime}=\mathcal{O}_{H}\left(N_{H}(\sigma)\right)-\{H\}$ with $\mathcal{F}(H)$.

The proofs of Propositions 8 and 9 appear in Section 8.

Proposition 8. Assume that $M \in \mathcal{O}_{G}(H)^{\prime}$ is doubled. Then one of the following statements holds:

(1) $H$ is doubled and $F^{*}(M)=F^{*}(H)$;

(2) $H$ is complemented and $F^{*}(M)=D C_{G}(D)$.

Proposition 9. Assume that $H$ is doubled and pick a component L of $H$. Let $M \in \mathcal{O}_{G}(H)^{\prime}$. Then one of the following statements holds:

(1) $M$ is doubled and $F^{*}(M)=F^{*}(H)$;

(2) $M$ is semisimple, $L$ is contained in a component of $M, H$ is transitive on the components of $M$, and the map $K \mapsto \mathcal{F}(H, K)$ is a bijection of $\mathcal{O}_{H}\left(N_{H}(L)\right)^{\prime}$ with $\mathcal{F}(H)$;

(3) $M$ is diagonal, $F^{*}(M)=F^{*}(H)$, and $M$ has $2|\Sigma(M)|$ components.

Proposition 10. Assume that $M \in \mathcal{O}_{G}(H)^{\prime}$ is complemented. Then $H$ is complemented and $F^{*}(M)=F^{*}(H)$.

Proposition 10 follows from Lemma 4.5.

Proposition 11. Assume that $H$ is complemented and pick a component $L$ of $H$. Let $M \in \mathcal{O}_{G}(H)^{\prime}$. Then one of the following statements holds: 
(1) $M$ is complemented and $F^{*}(M)=F^{*}(H)$;

(2) $M$ is semisimple, $L$ is contained in a component of $M, H$ is transitive on the components of $M$, and the map $K \mapsto \mathcal{F}(H, K)$ is a bijection of $\mathcal{O}_{H}\left(N_{H}(L)\right)^{\prime}$ with $\mathcal{F}(H)$;

(3) $\quad M$ is diagonal, $F^{*}(M)=D C_{G}(D), H$ has $|\Sigma(M)|$ components, and $M$ has $2|\Sigma(M)|$ components;

(4) $M$ is doubled and $F^{*}(M)=D C_{G}(D)$.

The proof of Proposition 11 appears in Section 8.

We are interested in the maximal overgroups of $H$ in $G$. Unless $H \leq A$ and $G=S$, $\mathcal{M}(H)$ is the set of maximal overgroups of $H$ in $G$. Moreover, the set $\mathcal{M}(H)$ can be retrieved from our propositions. For example, if $H$ is affine then by Proposition 4, Lemma 2.5, and Remark 2.7, $\mathcal{M}(H)=\left\{N_{G}(D), N_{G}(\mathcal{F}(\mathcal{D})) \mid \mathcal{D} \in \mathcal{D}(H)\right\}$. Similarly, if $H$ is semisimple and product indecomposable, but not octal or almost simple, then by Proposition 5, $\mathcal{M}(H)=\left\{N_{G}(\mathcal{F}) \mid \mathcal{F} \in \mathcal{F}(H)\right\}$, and $\mathcal{F}(H)$ is parameterized by $\mathcal{O}_{H}\left(N_{H}(L)\right)^{\prime}$, for $L$ a component of $H$.

Overgroup lattices The question of whether each nonempty finite lattice is isomorphic to an interval in the lattice of subgroups of some finite group has been of interest for at least 25 years since the appearance of [PP]. To illustrate how our propositions can be used to investigate this question and the lattice of subgroups of $S$, we also include two results which give information about $\mathcal{O}_{G}(H)$ when $H$ is the intersection of some (or many) pair(s) of maximal subgroups of $G$.

First, we (essentially) determine the pairs $M_{1}, M_{2}$ of distinct maximal subgroups of $G$ such that $M_{1} \cap M_{2}$ is primitive on $\Omega$.

THEOREM 12. Assume that $M_{1}$ and $M_{2}$ are distinct subgroups of $G$ maximal subject to $F^{*}(G) \not M_{i}$, such that $H=M_{1} \cap M_{2}$ is primitive. Assume that $|\Omega|$ is not prime. Then one of the following statements holds:

(1) $M_{1}, M_{2}$, and $H$ are almost simple;

(2) $\quad M_{i}=N_{G}\left(\mathcal{F}_{i}\right)$ for some regular product structures $\mathcal{F}_{1}$ and $\mathcal{F}_{2}$;

(3) interchanging $M_{1}$ and $M_{2}$ if necessary, $M_{1}$ and $H$ are affine with $F^{*}(H)=$ $F^{*}\left(M_{1}\right), M_{2}$ is semisimple, and there exists $\mathcal{D}=\left(D_{1}, \ldots, D_{k}\right) \in \mathcal{D}(H)$ such that $M_{2}=N_{G}(\mathcal{F}(\mathcal{D}))$ and $H=N_{M_{1}}(\mathcal{D})$;

(4) $H$ is octal semisimple and the wreath product of $L_{3}(2)$ by $S_{k},|\Omega|=8^{k}$, and $M_{1}$ and $M_{2}$ are the stabilizers of the two $H$-invariant affine structures on $\Omega$.

The proof of Theorem 12 appears in Section 9.

Next we impose a much stronger constraint on the lattice $\mathcal{O}_{G}(H)$.

THEOREM 13. Assume that $H$ is a proper primitive subgroup of $G$ and let $\mathcal{M}$ denote the set of maximal overgroups of $H$ in $G$. Assume that $|\Omega|$ is not prime and that,

for each $M \in \mathcal{M}$, there exists $M^{\prime} \in \mathcal{M}-\{M\}$ such that $H=M \cap M^{\prime}$.

Then one of the following statements holds: 
(1) $\mathcal{M}=\left\{N_{G}(\mathcal{F}) \mid \mathcal{F} \in \mathcal{F}(H)\right\}$;

(2) all members of $\mathcal{O}_{G}(H)$ are almost simple, product indecomposable, and not octal;

(3) $|\Omega|$ is a prime power, $H$ is affine, and $\mathcal{M}=\left\{N_{G}(D), N_{G}(\mathcal{F}(\mathcal{D})) \mid \mathcal{D} \in \mathcal{D}(H)\right\}$;

(4) $|\Omega|=8, G=A, H \cong L_{3}(2)$ is octal, and $\mathcal{M}$ consists of the stabilizers of the two $H$-invariant affine structures on $\Omega$;

(5) $|\Omega|=8, \quad G=S, \quad H \cong L_{3}(2)$ is octal, $\quad N_{G}(H) \cong P G L_{2}(7), \quad \mathcal{M}=\left\{F^{*}(S)\right.$, $\left.N_{G}(H)\right\}$, and $\mathcal{O}_{G}(H)=\mathcal{M} \cup\left\{H, K_{1}, K_{2}, G\right\}$, where $K_{1}$ and $K_{2}$ are the stabilizers of the two $H$-invariant affine structures on $\Omega$;

(6) $G=S, N_{G}(H)$ is the stabilizer in $S$ of an affine structure, regular product structure, or diagonal structure on $\Omega, H$ is the stabilizer in A of that structure, $\mathcal{M}=\left\{A, N_{S}(H)\right\}$, and $\mathcal{O}_{G}(H)=\left\{H, A, N_{S}(H), S\right\}$.

The proof of Theorem 13 appears in Section 9.

One can (with some difficulty) determine the groups $H$ and the lattice $\mathcal{O}_{G}(H)$ appearing in case (2) of Theorem 13 from the lists of inclusions among almost simple primitive groups appearing in [LPS1]. The analysis of case (1) of Theorem 13 would seem to demand some machinery to analyze the relationship among regular product structures on $\Omega$. Such machinery and a treatment of cases (1), (2), and (3) appear in a sequel to this work. In particular, Theorem 13 is extended there to give an explicit description of those primitive subgroups $H$ of $S$ such that the lattice $\mathcal{O}_{G}(H)$ satisfies condition (0.1) of Theorem 13.

A finite lattice $\Lambda$ has a greatest element $\infty$ and least element 0 , and we can view $\Lambda^{\prime}=\Lambda-\{0, \infty\}$ as a graph with adjacency relation the comparability relation. Define $\Lambda$ to be disconnected if the graph $\Lambda^{\prime}$ is disconnected. We observe that if $H$ is a primitive subgroup of $G$ such that the lattice $\mathcal{O}_{G}(H)$ is disconnected, then $\mathcal{O}_{G}(H)$ satisfies hypothesis (0.1) of Theorem 13. In particular, Theorem 13 is part of a program of J. Shareshian and the author (see [A3] and [A4]) to study interval lattices $\mathcal{O}_{U}(V)$ in finite groups $V$ over $U \leq V$, and indeed to prove the following statement:

(D) There exists a class of disconnected finite lattices, such that no member of the class is an interval in the lattice of subgroups of any finite group.

In the Aschbacher-Shareshian program, the class of lattices considered consists of those disconnected lattices $\Lambda$ with the property that, for each connected component $C$ of $\Lambda, C$ is the poset of nonempty proper subsets of a set of order $m(C)$, for some $m(C)>2$.

Define a lattice $\Lambda$ to be an $M$-lattice if $\Lambda^{\prime}$ has no edges. There is also a program in the literature to show that (D) is satisfied by some subclass of the class of $M$-lattices. Perhaps the major result in that program is due to Baddeley and Lucchini [BL], and reduces the problem for $M$-lattices to various problems involving almost simple groups. One of those problems is to show that there exists an $M$-lattice which is not of the form $\mathcal{O}_{U}(V)$ for any almost simple group $U$ and subgroup $V$. The extension of Theorem 13 mentioned above establishes this fact when $U$ is alternating or symmetric, and $V$ is primitive. 


\section{Some structures on a set}

In this section we assume that $\Omega$ is a finite set and let $S=\operatorname{Sym}(\Omega)$ be the symmetric group on $\Omega$. We recall some structures on $\Omega$ which will be important in this paper.

DEFINITION 1.1. Let $a$ be a positive integer and write $\Omega^{a}$ for the set product of $a$ copies of $\Omega$. Given an $a$-ary relation $R \subseteq \Omega^{a}$ on $\Omega$, define the stabilizer in $S$ of $R$ to be the subgroup $N_{S}(R)$ of $S$ consisting of those $g \in S$ such that $R g=R$.

DEFINITION 1.2. Assume that $|\Omega|=p^{e}$ is a power of a prime $p$. An affine structure on $\Omega$ is a quaternary relation $R=R(\Omega,+$ ) on $\Omega$ of the form

$$
R=\{(a, b, c, b+c-a) \mid a, b, c \in \Omega\},
$$

defined by some $e$-dimensional vector space structure $(\Omega,+)$ on $\Omega$ over $\mathbf{F}_{p}$.

The following results are well known.

LEMMA 1.3. Let $V=(\Omega,+)$ be an e-dimensional vector space structure on $\Omega$ over $\mathbf{F}_{p}, R=R(V)$ the corresponding affine structure on $\Omega, M=N_{S}(R)$ the stabilizer of $R$, and $0 \in \Omega$ the zero of $V$. Then:

(1) $F^{*}(M)=D \cong E_{p^{e}}$ is the group of translations $\tau_{a}: b \mapsto a+b, a \in \Omega$, on $V$, and $D$ is regular on $\Omega$;

(2) $\quad M_{0} \cong G L(V)$ and the map $a \mapsto \tau_{a}$ is an equivalence of the representation of $M_{0}$ on $\Omega$ with the representation of $M_{0}$ on $D$ via conjugation.

LEMMA 1.4. Assume that $p$ is a prime and that $E_{p^{e}} \cong D$ is a subgroup of $S$ regular on $\Omega$. Pick $\omega \in \Omega$ and define

$$
R=R(D)=\left\{\left(\omega a, \omega b, \omega c, \omega b c a^{-1}\right) \mid a, b, c \in D\right\} .
$$

Then $R$ is an affine structure on $\Omega$ (independent of $\omega$ ) with translation group $D$, and $N_{S}(R)=N_{S}(D)$.

DEFINITION 1.5. Let $m, k$ be integers with $m \geq 5$ and $k>1$. We recall the notion of a regular $(m, k)$-product structure on $\Omega$. Informally, such a structure is a bijection $f: \Omega \rightarrow \Gamma^{I}$, where $I=\{1, \ldots, k\}$ and $\Gamma$ is an $m$-set. The function $f$ may be thought of as a family of functions $\left(f_{i}: \Omega \rightarrow \Gamma: i \in I\right)$ via $f(\omega)=\left(f_{1}(\omega), \ldots, f_{k}(\omega)\right)$ for $\omega \in \Omega$.

Formally, a product structure is a family $\mathcal{F}=\left(\Omega_{i}: i \in I\right)$ of partitions $\Omega_{i}$ of $\Omega$ into $m$ blocks of size $m^{k-1}$, such that $\mathcal{F}$ is injective: for each pair of distinct points $\omega$, $\omega^{\prime}$ of $\Omega, \mathcal{F}(\omega) \neq \mathcal{F}\left(\omega^{\prime}\right)$, where $\mathcal{F}(\omega)$ is the family $\left(B_{i}: i \in I\right)$ of blocks defined by $\omega \in B_{i} \in \Omega_{i}$.

The family $\mathcal{F}=\mathcal{F}(f)$ defined by $f$ has $i$ th partition $\Omega_{i}=\left\{f_{i}^{-1}(\gamma) \mid \gamma \in \Gamma\right\}$, the fibers of $f_{i}$. An indexing of $\mathcal{F}$ is an indexing $\Omega_{i}=\left\{\Omega_{i, \gamma} \mid \gamma \in \Gamma\right\}$ of the blocks of the various partitions $\Omega_{i}$. The function $f$ defines the indexing $\Omega_{i, \gamma}=f_{i}^{-1}(\gamma)$, while an indexing of $\mathcal{F}$ defines a function $f$ via $\omega \in \Omega_{i, f_{i}(\omega)}$. As $\mathcal{F}$ is injective, the function 
$f$ defined by the indexing is injective, so as $|\Omega|=\left|\Gamma^{I}\right|, f: \Omega \rightarrow \Gamma^{I}$ is a bijection. In short, the formal definition is a 'coordinate-free' definition of product structure.

The stabilizer $N_{S}(\mathcal{F})$ in $S$ of $\mathcal{F}$ is the subgroup consisting of those $g \in S$ such that $\mathcal{F} g=\mathcal{F}$. Write $\mathcal{F}=\mathcal{F}(\Omega)$ for the set of all regular product structures on $\Omega$.

The referee has pointed out that the description above of a product structure as a chamber system is also used in [BP], where product structures are called Cartesian decompositions, and various properties of Cartesian decompositions are established.

EXAmple 1.6. Assume that $|\Omega|=m^{k}$ and let $\omega \in \Omega$ and $I=\{1, \ldots, k\}$. Assume that $D$ is a transitive subgroup of $S$ which is the direct product of a set $\mathcal{D}=\left\{D_{i} \mid i \in I\right\}$ of subgroups such that

$$
D_{\omega}=\prod_{i \in I} D_{i, \omega},
$$

with $\left|D_{i}: D_{i, \omega}\right|=m$ for each $i \in I$. Define $\mathcal{F}=\mathcal{F}(\mathcal{D})=\left(\Omega_{i} \mid i \in I\right)$ to be the product structure on $\Omega$ such that $\Omega_{i}$ is the set of orbits of $D_{i^{\prime}}$ on $\Omega$, where $D_{i^{\prime}}=\left\langle D_{j}\right| j$ $\in I-\{i\}\rangle$. It is well known that $\mathcal{F}$ is a product structure, and that if $G$ is a subgroup of $S$ permuting $\mathcal{D}$ via conjugation then $G \leq N_{S}(\mathcal{F})$.

LEMMA 1.7. Assume the setup of Example 1.6, and assume for each $i \in I$ that $E_{i} \leq D_{i}$ with $D_{i}=D_{i, \omega} E_{i}$. Set $\mathcal{E}=\left\{E_{i} \mid i \in I\right\}$. Then $\mathcal{F}(\mathcal{D})=\mathcal{F}(\mathcal{E})$.

Proof. As $D_{i}=D_{i, \omega} E_{i}$ for each $i \in I, \omega D_{i^{\prime}}=\omega E_{i^{\prime}}$ and $\Omega_{i}$ is the set of orbits of $E_{i^{\prime}}$ on $\Omega$.

LEMMA 1.8. Let $\mathcal{F}=\left(\Omega_{i}: i \in I\right)$ be a regular $(m, k)$-product structure on $\Omega$, pick $\omega \in \Omega$, and set $M=N_{S}(\mathcal{F})$.

(1) $F^{*}(M)=D=D_{1} \times \cdots \times D_{k}$, where, for $i \in I, D_{i}$ acts faithfully on $\Omega_{i}$ as the alternating group, and $D_{i^{\prime}}$ acts transitively on each block in $\Omega_{i}$.

(2) $\mathcal{F}=\mathcal{F}(\mathcal{D})$, where $\mathcal{D}=\left\{D_{i} \mid i \in I\right\}$.

(3) $M$ is isomorphic to the wreath product of $S_{m}$ by $S_{k}$. More precisely, $D \leq \bar{D} \unlhd M$ with $\bar{D}=\bar{D}_{1} \times \cdots \times \bar{D}_{k}, F^{*}\left(\bar{D}_{i}\right)=D_{i}, \bar{D}_{i}$ acts trivially on $\Omega_{j}$ for $j \neq i$, and faithfully on $\Omega_{i}$ as the symmetric group, and there is a complement $T$ to $\bar{D}$ in $M$ contained in $M_{\omega}$ which acts faithfully as the symmetric group on $\overline{\mathcal{D}}=\left\{\bar{D}_{i} \mid i \in I\right\}$ via conjugation, with $N_{T}\left(\bar{D}_{i}\right)$ centralizing $\bar{D}_{i}$.

PROOF. This is part of the folklore and the proof is straightforward.

Definition 1.9. Assume that $|\Omega|=c^{k-1}$ for some integer $k>1$ and some nonabelian finite simple group $L$ of order $c$, and let $\omega \in \Omega$. Assume that $D=D_{1} \times \cdots \times D_{k}$ is a transitive subgroup of $S$ which is the direct product of a set $\mathcal{D}=\left\{D_{i} \mid i \in I\right\}$ of subgroups isomorphic to $L$, and such that $D_{\omega}$ is a full diagonal subgroup $F$ of $D$ with respect to the direct product decomposition. (see [AS, Section 1] for a discussion of full diagonal subgroups.) Then $\mathcal{D}$ and $F$ define a 
diagonal structure $\mathbf{d}=\operatorname{diag}(\mathcal{D}, F)$ on $\Omega$, whose stabilizer $N_{S}(\mathbf{d})$ in $S$ we decree to be the subgroup $D\left(N_{S}(D) \cap N_{S}(F)\right)$.

The following lemma is part of the folklore and easy to prove.

LEMMA 1.10. Let $\mathbf{d}=\operatorname{diag}(\mathcal{D}, F)$ be a diagonal structure on $\Omega, \omega \in \Omega$, and set $M=N_{S}(\mathbf{d})$.

(1) $F^{*}(M)=D$ and $M=D M_{\omega}$.

(2) $\quad M_{\omega}=K T$ where $T=C_{M}(F)$ acts faithfully as the symmetric group on $\mathcal{D}$ via conjugation, $K$ is the kernel of the action of $M_{\omega}$ on $\mathcal{D}, F=F^{*}(K)$, and $K \cong \operatorname{Aut}(F)$.

We next define a notion of composition of regular product structures.

Definition 1.11. Let $m, k, \hat{m}, \hat{k}$ be integers with $m, \hat{m} \geq 5$ and $k, \hat{k}>1$. Let $I=\{1, \ldots, k\}, \hat{I}=\{1, \ldots, \hat{k}\}$, and let $\Gamma$ be an $m$-set and $\hat{\Gamma}$ an $\hat{m}$-set. Let $\mathcal{F}=$ $\left(\Omega_{i}: i \in I\right)$ be a regular $(m, k)$-product structure on $\Omega$ and $\hat{\mathcal{F}}=\left(\Gamma_{j}: j \in \hat{I}\right)$ be a regular $(\hat{m}, \hat{k})$-product structure on $\Gamma$. Recall from Definition 1.5 that we can choose bijections $f: \Omega \rightarrow \Gamma^{I}$ and $\hat{f}: \Gamma \rightarrow \hat{\Gamma}^{\hat{I}}$ so that $\mathcal{F}=\mathcal{F}(f)$ and $\hat{\mathcal{F}}=\mathcal{F}(\hat{f})$. That is, $f(\omega)=\left(f_{1}(\omega), \ldots, f_{k}(\omega)\right)$ and $\Omega_{i}=\left\{f_{i}^{-1}(\gamma) \mid \gamma \in \Gamma\right\}$, and similarly for $\hat{f}$. Define $\tilde{m}=\hat{m}, \tilde{\Gamma}=\hat{\Gamma}$, and $\tilde{I}=I \times \hat{I}$. Thus $\tilde{k}=|\tilde{I}|=k \hat{k}$. Define

$$
\tilde{f}: \Omega \rightarrow \tilde{\Gamma}^{\tilde{I}}
$$

by $\tilde{f}(\omega)=\left(\hat{f}\left(f_{1}(\omega), \ldots, \hat{f}\left(f_{k}(\omega)\right)\right)\right)$, for $\omega \in \Omega$. That is, $\tilde{f}=\left(\tilde{f}_{i, j}:(i, j) \in \tilde{I}\right)$ has coordinate functions $\tilde{f}_{i, j}=\hat{f}_{j} \circ f_{i}$ for $(i, j) \in \tilde{I}$.

Visibly $\tilde{f}$ is an informal regular $(\tilde{m}, \tilde{k})$-product structure on $\Omega$, as defined in Definition 1.5, giving rise to the formal product structure $\tilde{\mathcal{F}}=\mathcal{F}(\tilde{f})=\left\{\Omega_{i, j} \mid(i, j) \in\right.$ $\tilde{I}\}$, where $\Omega_{i, j}=\left\{f_{i, j}^{-1}(\alpha) \mid \alpha \in \tilde{\Gamma}\right\}$. We call $\tilde{\mathcal{F}}$ a composition of $\hat{\mathcal{F}}$ and $\mathcal{F}$, and sometimes write $\hat{\mathcal{F}} \circ \mathcal{F}$ for such a composition.

Alternatively, as in Definition 1.5, pick indexings $\Omega_{i}=\left\{\Omega_{i, \gamma} \mid \gamma \in \Gamma\right\}$ and $\Gamma_{j}=$ $\left\{\Gamma_{j, \alpha} \mid \alpha \in \hat{\Gamma}\right\}$, and for $(i, j) \in \tilde{I}$ define

$$
\Omega_{i, j}=\left\{\Omega_{i, j, \alpha} \mid \alpha \in \tilde{\Gamma}\right\} \quad \text { where } \Omega_{i, j, \alpha}=\bigcup_{\gamma \in \Gamma_{j, \alpha}} \Omega_{i, \gamma}
$$

Then $\tilde{\mathcal{F}}=\left(\Omega_{i, j} \mid(i, j) \in \tilde{I}\right)$ is a regular $(\tilde{m}, \tilde{k})$-product structure on $\Omega$ and a composition of $\hat{\mathcal{F}}$ with $\mathcal{F}$.

LEMMA 1.12. Let $m, k, \hat{m}, \hat{k}$ be integers with $m, \hat{m} \geq 5$ and $k, \hat{k}>1$. Let $I=\{1, \ldots, k\}, \hat{I}=\{1, \ldots, \hat{k}\}$, and $\mathcal{F}=\left(\Omega_{i} \mid i \in I\right)$ be a regular $(m, k)$-product 
structure on $\Omega$. Assume for each $i \in I$ that $\hat{\mathcal{F}}_{i}=\left(\Delta_{i, j} \mid j \in \hat{I}\right)$ is a regular $(\hat{m}, \hat{k})$-product structure on $\Omega_{i}$. Define $\tilde{I}=I \times \hat{I}$, and $\tilde{\mathcal{F}}=\left(\Omega_{i, j} \mid(i, j) \in \tilde{I}\right)$, where

$$
\Omega_{i, j}=\left\{\tilde{B} \mid B \in \Delta_{i, j}\right\} \quad \text { and } \quad \tilde{B}=\bigcup_{A \in B} A .
$$

(1) Then $\tilde{\mathcal{F}}$ is a composition $\hat{\mathcal{F}}_{1} \circ \mathcal{F}$ of the regular product structures $\hat{\mathcal{F}}_{1}$ and $\mathcal{F}$.

(2) Assume that $H \leq N_{S}(\mathcal{F})$ permutes $\left\{\hat{\mathcal{F}}_{i} \mid i \in I\right\}$. Then $H \leq N_{S}(\tilde{\mathcal{F}})$.

Proof. Let $\hat{\mathcal{F}}=\hat{\mathcal{F}}_{1}, \Gamma=\Omega_{1}$, and $\Gamma_{j}=\Delta_{1, j}$ for $j \in \hat{I}$. As all regular $(\hat{m}, \hat{k})$-product structures are isomorphic, for each $i \in I$ there exists an isomorphism $\varphi_{i}: \hat{\mathcal{F}} \rightarrow \hat{\mathcal{F}}_{i}$ with $\Gamma_{j} \varphi_{i}=\Delta_{i, j}$ for $j \in \hat{I}$. Take $\varphi_{1}=1$.

For $\gamma \in \Gamma$ and $i \in I$, set $\Omega_{1, \gamma}=\gamma$ and $\Omega_{i, \gamma}=\gamma \varphi_{i}$. Thus $\Omega_{i}=\left\{\Omega_{i, \gamma} \mid \gamma \in \Gamma\right\}$ is an indexing of $\mathcal{F}$. Let $\hat{\Gamma}$ be an $\hat{m}$-set and $\Gamma_{j}=\left\{\Gamma_{j, \alpha} \mid \alpha \in \hat{\Gamma}\right\}, j \in \hat{I}$, be an indexing of $\hat{\mathcal{F}}$. For $(i, j) \in \tilde{I}$ and $\alpha \in \hat{\Gamma}$, set $\Delta_{i, j, \alpha}=\Gamma_{j, \alpha} \varphi_{i}$. Then

$$
\Delta_{i, j}=\Gamma_{j} \varphi_{i}=\left\{\Gamma_{j, \alpha} \varphi_{i} \mid \alpha \in \hat{\Gamma}\right\}=\left\{\Delta_{i, j, \alpha} \mid \alpha \in \hat{\Gamma}\right\},
$$

and we can form

$$
\tilde{\Delta}_{i, j, \alpha}=\bigcup_{A \in \Delta_{i, j, \alpha}} A
$$

as in (1.1). Set $\Omega_{i, j, \alpha}=\tilde{\Delta}_{i, j, \alpha}$. For $A \in \Delta_{i, j, \alpha}, A=\gamma \varphi_{i}$ for some $\gamma \in \Gamma_{j, \alpha}$, so $A=\gamma \varphi_{i}=\Omega_{i, \gamma}$. Thus

$$
\Omega_{i, j, \alpha}=\tilde{\Delta}_{i, j, \alpha}=\bigcup_{A \in \Delta_{i, j, \alpha}} A=\bigcup_{\gamma \in \Gamma_{j, \alpha}} \Omega_{i, \gamma}
$$

and by (1.1), (1.2), and (1.3),

$$
\Omega_{i, j}=\left\{\tilde{\Delta}_{i, j, \alpha} \mid \alpha \in \hat{\Gamma}\right\}=\left\{\Omega_{i, j, \alpha} \mid \alpha \in \hat{\Gamma}\right\} .
$$

Hence (1) follows from (1.3), (1.4), and Definition 1.11.

Assume the hypothesis of (2). As $H \leq N_{S}(\mathcal{F})$, for $h \in H$ and $i \in I$, we can define $i h \in I$ by $\Omega_{i} h=\Omega_{i h}$, to obtain a representation of $H$ on $I$. As $H$ permutes the $\hat{\mathcal{F}}_{i}$, for $(i, j) \in \tilde{I}, \Delta_{i, j} h=\Delta_{i h, j^{\prime}}$ for some $j^{\prime} \in \hat{I}$. Thus for $B \in \Delta_{i, j}, B h \in \Delta_{i h, j^{\prime}}$, so

$$
\tilde{B} h=\left(\bigcup_{A \in B} A\right) h=\bigcup_{A \in B} A h=\tilde{C},
$$

where $C=B h \in \Delta_{i h, j^{\prime}}$. Thus

$$
\Omega_{i, j} h=\left\{\tilde{B} h \mid B \in \Delta_{i, j}\right\}=\left\{\tilde{C} \mid C \in \Delta_{i h, j^{\prime}}\right\}=\Omega_{i h, j^{\prime}},
$$

so indeed $H \leq N_{S}(\tilde{F})$. 
LEMMA 1.13. Assume $I, m, k, D, \mathcal{D}$ satisfy the hypothesis of Example 1.6 and set $\mathcal{F}=\mathcal{F}(\mathcal{D})$. Let $\hat{m}, \hat{k}$ be integers with $\hat{m} \geq 5$ and $\hat{k}>1$, and set $\hat{I}=\{1, \ldots, \hat{k}\}$. Assume for each $i \in I$ that $\hat{\mathcal{F}}_{i}=\left(\Sigma_{i, j}: j \in \hat{I}\right)$ is a regular $(\hat{m}, \hat{k})$-product structure on $\Sigma_{i}=\omega D_{i}$. Define $\tilde{I}=I \times \hat{I}$, and $\tilde{\mathcal{F}}=\left(\Omega_{i, j}:(i, j) \in \tilde{I}\right)$, where

$$
\Omega_{i, j}=\left\{\tilde{U} \mid U \in \Sigma_{i, j}\right\} \quad \text { and } \quad \tilde{U}=\left\{u g \mid u \in U, g \in D_{i^{\prime}}\right\} .
$$

(1) Then $\tilde{\mathcal{F}}$ is a composition $\hat{\mathcal{F}}_{1} \circ \mathcal{F}$ of the regular product structures $\hat{\mathcal{F}}_{1}$ and $\mathcal{F}$.

(2) Assume that $D \unlhd H \leq S, D_{1}$ acts on $\hat{\mathcal{F}}_{1}$, and $H_{\omega}$ permutes the $\hat{\mathcal{F}}_{i}$. Then $H \leq N_{S}(\tilde{\mathcal{F}})$.

Proof. For $i \in I$ and $\sigma \in \Sigma_{i}$, set $\bar{\sigma}=\sigma D_{i^{\prime}} \subseteq \Omega$. Then for $U \subseteq \Sigma_{i}$, set $\bar{U}=\{\bar{u} \mid u \in$ $U\}$, and for $(i, j) \in \tilde{I}$, set $\Delta_{i, j}=\left\{\bar{U} \mid U \in \Sigma_{i, j}\right\}$. Now $\sigma=\omega x$ for some $x \in D_{i}$, so $\bar{\sigma}=\omega x D_{i^{\prime}}=\omega D_{i^{\prime}} x$, and hence the map $\psi_{i}: \Sigma_{i} \rightarrow \Omega_{i}$ defined by $\psi_{i}: \sigma \mapsto \bar{\sigma}$ is a bijection. In particular as $\hat{\mathcal{F}}_{i}$ is a regular $(\hat{m}, \hat{k})$-product structure on $\Sigma_{i}, \mathcal{E}_{i}=\left(\Delta_{i, j}\right.$ : $j \in \hat{I})$ is a regular $(\hat{m}, \hat{k})$-product structure on $\Omega_{i}$. Furthermore, for $U \in \Sigma_{i, j}$,

$$
\tilde{U}=\left\{u g \mid u \in U, g \in D_{i^{\prime}}\right\}=\bigcup_{u \in U} \bar{u}=\bigcup_{A \in \bar{U}} A,
$$

so $\tilde{U}$ is the set $\tilde{B}$ of (1.1) in Lemma 1.12 , for $B=\bar{U} \in \Delta_{i, j}$. Therefore $\Omega_{i, j}=\{\tilde{U} \mid$ $\left.U \in \Sigma_{i, j}\right\}$ is the set $\left\{\tilde{B} \mid B \in \Delta_{i, j}\right\}$ of (1.1), so (1) follows from Lemma 1.12(1).

Now assume the hypothesis of (2). As $D$ is transitive on $\Omega, H=D H_{\omega}$. As $D_{i}$ acts on $\hat{\mathcal{F}}_{i}$ and $\psi_{i}$ is $D_{i}$-equivariant, $D_{i}$ acts on $\mathcal{E}_{i}$. Furthermore, $D_{i^{\prime}}$ is trivial on $\Omega_{i}$, so $D$ acts on $\mathcal{E}_{i}$.

Next, as in the proof of Lemma 1.12, there is a representation of $H$ on $I$ such that $D_{i}^{h}=D_{i h}$. For $h \in H_{\omega}$ and $u \in \Sigma_{i}, u=\omega x$ for some $x \in D_{i}$, so $u h=\omega x h=\omega h x^{h}=$ $\omega x^{h} \in \Sigma_{i h}$. By hypothesis, $H_{\omega}$ permutes the $\hat{\mathcal{F}}_{i}$, so $\Sigma_{i, j} h=\Sigma_{i h, j^{\prime}}$ for some $j^{\prime} \in \hat{I}$. Then for $U \in \Sigma_{i, j}$,

$$
\bar{U} h=\left\{u D_{i^{\prime}} h \mid u \in U\right\}=\left\{u h D_{(i h)^{\prime}} \mid u \in U\right\}=\left\{v D_{(i h)^{\prime}} \mid v \in U h\right\}=\overline{U h},
$$

so $\Delta_{i, j} h=\Delta_{i h, j^{\prime}}$. That is, $H_{\omega}$ permutes the $\mathcal{E}_{i}$. Then as $H=D H_{\omega}, H$ permutes the $\mathcal{E}_{i}$, so (2) follows from Lemma 1.12(2).

\section{Primitive groups}

In this section we make the following assumption.

Hypothesis 2.1. $\Omega$ is a finite set, $S=\operatorname{Sym}(\Omega)$ is the symmetric group on $\Omega, \omega \in \Omega$, $G$ is $S$ or the alternating group $A$ on $\Omega, H$ is a subgroup of $G$ primitive on $\Omega$, and $D=F^{*}(H)$. 
LEMMA 2.2. $H=D H_{\omega}$ and one of the following statements holds.

(1) $|\Omega|=p^{e}$ is a power of the prime $p, D \cong E_{p^{e}}$ is regular on $\Omega$, and $H_{\omega}$ is a complement to $D$ in $H$ which is irreducible on $D$ regarded as an $\mathbf{F}_{p} H_{\omega}$-module.

(2) $D=D_{1} \times D_{2}, D_{1}$ and $D_{2}$ are isomorphic normal subgroups of $H, D_{i}$ is the direct product of $k$ isomorphic nonabelian simple components $L$ of $H$, permutated transitively by $H, D_{i}$ is regular on $\Omega, D_{\omega}$ is a full diagonal subgroup of $D$, and $H_{\omega}=N_{H}\left(D_{\omega}\right)$. Furthermore, $|\Omega|=|L|^{k}$.

(3) $D$ is the direct product of the set $\mathcal{L}$ of components of $H, H$ is transitive on $\mathcal{L}$, and choosing $L \in \mathcal{L}$, one of the following statements holds:

(i) $D_{\omega}$ is the direct product of the groups $F_{\omega}, F \in \mathcal{L}, L_{\omega} \neq 1$, and $\operatorname{Aut}_{H_{\omega}}(L)$ is maximal in $\operatorname{Aut}_{H}(L)$. Furthermore, $|\Omega|=\left|L: L_{\omega}\right|^{|\mathcal{L}|}$.

(ii) There exists a maximal $H$-invariant partition $\Sigma=\Sigma(H)$ of $\mathcal{L}$ such that $D_{\omega}$ is the direct product of full diagonal subgroups $F_{\sigma}$ of $D_{\sigma}=\langle\sigma\rangle, \sigma \in \Sigma$. Furthermore, $|\Omega|=|L|^{|\mathcal{L}|-|\Sigma|}$.

(iii) $\operatorname{Inn}(L) \leq \operatorname{Aut}_{H_{\omega}}(L)$ and $H_{\omega}$ is a complement to $D$ in $H$. Furthermore, $|\Omega|=|L|^{|\mathcal{L}|}$.

PROOF. This is a consequence of [AS, Theorem 1].

Definition 2.3. In case (1) of Lemma 2.2, we say that $H$ is affine. In case (2), we say that $H$ is doubled. In case (3i) we say that $H$ is semisimple, in case (3ii) we say that $H$ is diagonal, and in case (3iii) we say that $H$ is complemented. We say that $H$ is strongly diagonal if $H$ is diagonal and $\Sigma(H)=\{\mathcal{L}\}$.

In Praeger's theory [P] of overgroups in $S$ of primitive subgroups of $S$, a somewhat different partition of primitive groups appears. In Praeger's terminology, affine groups are of type $H A$, doubled groups are of type $H C$, semisimple but not almost simple groups are of type $P A$, diagonal groups are of type $C D$, complemented groups are of type $T W$, and strongly diagonal groups are of type $S D$.

LEMMA 2.4. Let $M \in \mathcal{O}_{G}(H)$.

(1) $M$ is primitive on $\Omega$.

(2) If $X \unlhd M$ and $1 \neq D \cap X$ then either $D \leq X$ or $H$ is doubled and $D_{i}=X \cap D$ for $i=1$ or 2 .

Proof. Part (1) is trivial. Part (2) follows from the fact that either $D$ is a minimal normal subgroup of $H$, or $H$ is doubled and $D_{1}$ and $D_{2}$ are the minimal normal subgroups of $H$.

LEMMA 2.5. Let $M$ be a maximal subgroup of $G$ primitive on $\Omega$ and $X=F^{*}(M)$. Then one of the following statements holds: 
(1) $\quad M$ is affine. That is, $|\Omega|=p^{e}$ is a power of the prime $p$, $M$ is the stabilizer of an affine structure on $\Omega, X \cong E_{p^{e}}$ is regular on $\Omega$, and $N_{S}(X)_{\omega}$ is a complement to $X$ in $N_{S}(X)$ which acts faithfully as $G L(D)$ on $D$ regarded as an e-dimensional $F_{p}$-space.

(2) $\quad M$ is the stabilizer of a regular $(m, k)$-product structure on $\Omega$, for some $m \geq 5$ and $k \geq 2, M$ is semisimple, $X$ is the direct product of $k$ copies of $A_{m},|\Omega|=\bar{m}^{k}$, and $N_{S}(X)$ is the wreath product of $S_{m}$ by $S_{k}$.

(3) $M$ is the stabilizer of a diagonal structure on $\Omega, X$ is the direct product of $k$ copies of some nonabelian simple group $Y, M$ is diagonal, $|\Omega|=|Y|^{k-1}$, and $N_{S}(X) / X \cong S_{k} \times \operatorname{Out}(Y)$.

(4) $\quad M$ is almost simple and $N_{S}(X)$ is the stabilizer in $\operatorname{Aut}(X)$ of the equivalence class of the representation of $X$ on $\Omega$.

Proof. This follows from the O'Nan-Scott theorem, which is in turn a fairly easy consequence of Lemma 2.2; see the Appendix to [AS]. The stabilizers of the structures in (1)-(3) are almost always maximal. See Remark 2.7 for more discussion.

Notation 2.6. Write $\mathcal{F}(H)$ for the set of $H$-invariant regular product structures on $\Omega$.

If $H$ is affine, write $\mathcal{D}(H)$ for the set of systems $\mathcal{D}=\left\{D_{1}, \ldots, D_{k}\right\}$ of imprimitivity for $H$ on $D$. That is, $k>1, D=D_{1} \times \cdots \times D_{k}$, and $H$ permutes $\mathcal{D}$ transitively via conjugation. Observe that for $\mathcal{D} \in \mathcal{D}(H), \mathcal{F}(\mathcal{D})$ is an $H$-invariant regular $(d, k)$-product structure on $\Omega$ by Example 1.6, where $d=\left|D_{i}\right|$.

In the remaining cases of Lemma 2.2, a minimal normal subgroup $E$ of $H$ is the direct product of its set $\mathcal{L}$ of components permutated transitively by $H$ via conjugation. Let $\mathcal{P}(H)$ be the set of $H$-invariant partitions $\Delta$ of $\mathcal{L}$ such that $|\Delta|>1$, and such that $\Sigma(H)$ is a refinement of $\Delta$ if $H$ is diagonal. Let $\Delta \in \mathcal{P}(H)$ and, for $\delta \in \Delta$, set $E_{\delta}=\langle\delta\rangle, k=|\Delta|$, and $m=\left|E_{\delta}: E_{\delta, \omega}\right|$. Define $\mathcal{D}(\Delta)=\left\{E_{\delta} \mid \delta \in \Delta\right\}$ and set $\mathcal{F}(H, \Delta)=\mathcal{F}(\mathcal{D}(\Delta))$ the $H$-invariant $(m, k)$-product structure on $\Omega$ supplied by Example 1.6.

Observe that we have a bijection $K \mapsto \gamma_{K}^{H}$ of $\mathcal{O}_{H}\left(N_{H}(U)\right)^{\prime}=\mathcal{O}_{H}\left(N_{H}(U)\right)-\{H\}$ with $\mathcal{P}(H)$, where $U$ is some fixed member of $\mathcal{L}$ and $\gamma_{K}=U^{K}$ if $H$ is doubled, semisimple, or complemented, while $U=E_{\sigma}$ for some fixed $\sigma \in \Sigma(H)$ and $\gamma_{K}=L^{K}$ for $L \in \sigma$ if $H$ is diagonal. We also write $\mathcal{F}(H, K)$ for $\mathcal{F}\left(H, \gamma_{K}^{H}\right)$.

Write $\mathcal{F}(H)$ for $\mathcal{F}\left(H, N_{H}(U)\right)$ if $H$ is doubled, semisimple, or complemented, and $|\mathcal{L}|>1$. Set $\mathcal{F}(H)=\mathcal{F}\left(H, N_{H}(\sigma)\right)$ if $H$ is diagonal but not strongly diagonal.

REMARK 2.7. Liebeck et al. [LPS1] determine when the subgroups listed in Lemma 2.5 are maximal in $G$. In particular, they supply an explicit list of those primitive almost simple groups which are not maximal.

One can also use our propositions to see that the stabilizers $H$ listed in the first three parts of Lemma 2.5 are almost always maximal. (It should be pointed out, however, that such a treatment of the maximality of the stabilizers still depends in part on [LPS1], since the proofs of some of these propositions sometimes make 
appeals to [LPS1].) Observe that if $G=S$ and $H<L=F^{*}(S)$, then $H$ is not maximal. As it is not difficult to determine when $H \leq L$, we ignore this subtlety in the following discussion. We also assume that $|\Omega|$ is not prime.

If $H$ is almost simple, product indecomposable, and not octal, then by Lemma 8.5, $\mathcal{M}(H)$ consists of almost simple groups which are product indecomposable and not octal. On the other hand, by Proposition 1, if $H$ is not almost simple then no member of $\mathcal{M}(H)$ is almost simple, so, in particular, maximal semisimple overgroups of $H$ are stabilizers of product structures.

If $H$ is affine then from Lemma 1.3, $H$ is imprimitive on $D$, so $\mathcal{D}(H)=\varnothing$. Therefore $H$ is maximal by Proposition 4 .

If $H$ is the stabilizer of a regular product structure, then the components $D_{i}$ of $H$ are alternating groups and, in particular, are product indecomposable and not octal. Furthermore, $H$ is primitive on its components, so $\mathcal{O}_{H}\left(N_{H}\left(D_{i}\right)\right)=\varnothing$. Therefore, $H$ is maximal by Proposition 5.

Finally, if $H$ is the stabilizer of a diagonal structure, then $\Sigma(H)=\{\mathcal{L}\}$, where $\mathcal{L}$ is the set of components of $H$. Therefore $H$ is maximal by Proposition 7 .

\section{Preliminary lemmas}

LEMMA 3.1. Assume that $p$ is a prime, $G$ is a nonabelian finite simple group, and $H<G$ with $|G: H|=p^{a}$. Then one of the following statements holds:

(a) $G \cong A_{p^{a}}$ and $H \cong A_{p^{a}-1}$;

(b) $G \cong L_{n}(q)$ for some prime power $q$ and some prime $n, H$ is the stabilizer of a point or a hyperplane of the projective geometry for $G$, and $p^{a}=\left(q^{n}-1\right)$ / $(q-1)$;

(c) $G \cong L_{2}(11), H \cong A_{5}$, and $p^{a}=11$;

(d) $\left(G, H, p^{a}\right)$ is $\left(M_{23}, M_{22}, 23\right)$ or $\left(M_{11}, M_{10}, 11\right)$;

(e) $\quad G \cong P S p_{4}(3) \cong U_{4}(2), H$ is a maximal 2-parabolic of $G$ which is an extension of $E_{16}$ by $A_{5}$, and $p^{a}=3^{3}$.

ProOF. This is [Gu, Theorem 1].

LEMMA 3.2. Assume that $p$ is a prime, $G$ is a nonabelian finite simple group, and $H<G$ with $|G: H|=p^{a}$. Let $\mathcal{Q}$ be the set of abelian complements to $H$ in $G$.

(1) $\mathcal{Q}=\varnothing$ if and only if either

(i) $q$ is a Mersenne prime, $p=2$, and $G \cong L_{2}(q)$; or

(ii) case (e) of Lemma 3.1 holds.

(2) If some member of $\mathcal{Q}$ is noncyclic then case (a) of Lemma 3.1 holds.

PROOF. Represent $G$ by right multiplication on $\Omega=G / H$. In case (a) of Lemma 3.1, there is a subgroup $Q \cong E_{p^{a}}$ regular on $\Omega$, so $Q \in \mathcal{Q}$ and the lemma holds in this case.

Suppose that case (b) of Lemma 3.1 holds. As $n$ is prime, Zsigmondy's theorem (see [Gu, 3.2]) says that either conclusion (1i) holds, or there is a prime divisor $r$ of $q^{n}-1$ 
which does not divide $q^{k}-1$ for any $k<n$. In the first case the Sylow 2-subgroups of $G$ are the complements to $H$ in $G$, and are nonabelian dihedral groups, so we may assume the second case holds. Then as $p^{a}=\left(q^{n}-1\right) /(q-1)$, it follows that $p=r$. Let $V$ be the natural module for $\hat{G}=G L_{n}(q)$ and $P$ a subgroup of $\hat{G}$ of order $p$. As $p$ does not divide $q^{k}-1$ for $k<n, P$ is irreducible on $V$ and $C_{\hat{G}}(P)$ is the multiplicative group of $\mathbf{F}_{q^{n}}$ acting by right multiplication on $V$ regarded as the additive group of $\mathbf{F}_{q^{n}}$. In particular, $C_{\hat{G}}(P)$ is cyclic, so $G$ has a cyclic Sylow $p$-subgroup $Q$ whose preimage $\hat{Q}$ in $\hat{G}$ is irreducible on $V$, and hence regular on the points and hyperplanes of $V$. Thus $Q$ is regular on $\Omega$ so the lemma holds in this case.

In cases (c) and (d), a Sylow $p$-subgroup of $G$ is of order $p$, and $\mathcal{Q}=\operatorname{Syl}_{p}(G)$, so the lemma holds.

Finally, in case (e), $G$ acts transitively on the set $\mathcal{A}$ of abelian subgroups of $G$ of order $3^{3}$, and for $A \in \mathcal{A}, A \cong E_{3^{3}}$ contains a member of each of the three conjugacy classes of subgroups of $G$ of order 3 . Thus as $3 \in \pi(H), A$ is not regular on $\Omega$, so again the lemma holds.

LEMMA 3.3. Assume that $p$ is a prime, let $n$ be a positive integer and $e=\log _{p}\left((n !)_{p}\right)$ the log of the p-part of $n !$.

(1) If $n$ is a power of $p$ then $e=(n-1) /(p-1)$.

(2) $e \leq(n-1) /(p-1)$, with $n$ a power of $p$ in the case of equality.

PROOF. Part (1) is [A1, Lemma 7.1]. Let $n=\sum_{i} a_{i} p^{i}$ be the $p$-ary expansion of $n$. By [A1, Lemma 7.2],

$$
e=\sum_{i} a_{i} \log _{p}\left(\left(\left(p^{i}\right) !\right)_{p}\right)
$$

Thus if $n$ is not a power of $p$ then by induction on $n$,

$$
e \leq \sum_{i} a_{i}\left(p^{i}-1\right) /(p-1)=(n-r) /(p-1),
$$

where $r=\sum_{i} a_{i}>1$. Hence we may assume $n$ is a power of $p$, where (1) completes the proof.

LEMMA 3.4. Assume that $G$ is an almost simple group and set $L=F^{*}(G)$. Assume that $M$ is a maximal subgroup of $G$ not contained in $L$ and $A<L$ such that $L \cap M<A$ and

$$
L=A A^{\prime} \quad \text { where } A^{\prime}=\bigcap_{D \in A^{M}-\{A\}} D .
$$

Then $v=\left|M: N_{M}(A)\right|=2$ and, taking $B \in A^{M}-\{A\}$ and setting $U=A \cap B$, we obtain $U=L \cap M, M=N_{G}(U)$, and one of the following statements holds: 
(1) $L \cong A_{6}, G \cong \operatorname{Aut}(L), P G L_{2}(9)$, or $M_{10}, A \cong A_{5}, U \cong D_{10}$, and $M \cong \mathbf{Z}_{2} \times F_{20}$, $D_{20}$, or $F_{20}$, respectively, where $F_{20}$ is the Frobenius group of order 20;

(2) $L \cong M_{12}, G \cong \operatorname{Aut}\left(M_{12}\right), A \cong M_{11}, U \cong L_{2}(11)$, and $M \cong P G L_{2}(11)$;

(3) $L \cong S p_{4}(q)$ for some $q>2$ even, $G$ is a subgroup of $\operatorname{Aut}(L)$ acting nontrivially on the Dynkin diagram of $L, A \cong O_{4}^{-}(q)$, and $U=N_{A}(T)$ is an extension of $T \cong \mathbf{Z}_{q^{2}+1}$ by $\mathbf{Z}_{4}$.

PROOF. This lemma is essentially contained in [LPS1, proof of 4.3]. Furthermore, the referee observes that [BPS1, Lemma 5.2] can be used to give a short proof of the lemma.

Let $M_{L}=M \cap L$. As $M$ is maximal in $G$ and not contained in $L, G=M L$, $M=N_{G}\left(M_{L}\right)$, and $\mathcal{I}_{L}^{*}(M)=\left\{M_{L}\right\}$, where $\mathcal{I}_{L}^{*}(M)$ denotes the maximal members of the set of $M$-invariant proper subgroups of $L$. Then as $M_{L}<A<L$, we have $v=\left|M: N_{M}(A)\right|>1$. Let $m \in M-N_{M}(A)$ and set $B=A^{m}$ and $U=A \cap B$. Thus $M_{L} \leq U$, and by hypothesis (3.1), $L=A B$. Therefore $|U|=|A||B| /|L|=|A|^{2} /|L|$.

Let $A_{1} \in \mathcal{M}_{L}(A)$, and set $B_{1}=A_{1}^{m}$ and $U_{1}=A_{1} \cap B_{1}$. Set $X=\operatorname{Aut}(L)$ and $Y=L N_{X}\left(A_{1}\right)$.

As $L=A B$ with $A \leq A_{1}$ and $B \leq B_{1}$, also $L=A B_{1}=B A_{1}$. Hence $A_{1}=A_{1} \cap$ $A B_{1}=A\left(A_{1} \cap B_{1}\right)=A U_{1}$, and similarly $B_{1}=B U_{1}$. Similarly, $B_{1}=B_{1} \cap A B=$ $B\left(B_{1} \cap A\right)=B\left(U_{1} \cap A\right)$. Then also $U_{1}=U_{1} \cap B_{1}=U_{1} \cap B\left(U_{1} \cap A\right)=U_{A} U_{B}$, where $U_{A}=U_{1} \cap A$ and $U_{B}=U_{1} \cap B$.

Let $\alpha=\left|A_{1}: A\right|$. Then as $A_{1}^{m}=B_{1}$ and $A^{m}=B$, also $\alpha=\left|B_{1}: B\right|$. As $A_{1}=A U_{1}$, then $\left|U_{1}: U_{A}\right|=\alpha$, and similarly $\left|U_{1}: U_{B}\right|=\alpha$.

Next $L=A_{1} B_{1}$ is a maximal factorization, and hence is described in the main theorem of [LPS2]. Inspecting the tables of examples in that theorem for pairs ( $A_{1}, B_{1}$ ) with $B_{1} \cong A_{1}$, we conclude that one of the following statements holds:

(i) $L \cong A_{6}$ and $A_{1} \cong A_{5}$;

(ii) $L \cong M_{12}$ and $A_{1} \cong M_{11}$;

(iii) $L \cong S p_{4}(q)$ with $q>2$ even, and $A_{1} \cong O_{4}^{-}(q)$;

(iv) $L \cong P \Omega_{8}^{+}(q)$ and $A_{1} \cong \Omega_{7}(q)$.

Example (iii) appears in [LPS2, Table 1], using the fact that $\operatorname{Sp}_{2}\left(q^{2}\right) .2 \cong O_{4}^{-}(q)$. In example (iv), $\Omega_{7}(q)=S_{6}(q)$ when $q$ is even. Using the formula $|L|=\left|A_{1}\right|^{2} /\left|U_{1}\right|$, we calculate $\left|U_{1}\right|$ and then from the subgroup structure of $A_{1}$ we conclude that $U_{1} \cong D_{10}, L_{2}(11)$, an extension of $\mathbf{Z}_{q^{2}+1}$ by $\mathbf{Z}_{4}, G_{2}(q)$, in cases (i)-(iv), respectively.

Suppose that $A \neq A_{1}$. Then $\alpha=\left|A_{1}: A\right|>1$ and we showed above that $U_{1}=U_{A} U_{B}$, with

$$
\left|U_{1}: U_{C}\right|=\alpha \quad \text { for } C \in\{A, B\}
$$

In the first two cases this is impossible, as $U_{1}$ does not have a proper subgroup $U_{A}$ such that $\pi\left(U_{A}\right)=\pi\left(U_{1}\right)$. In the third case all Sylow subgroups of $U_{1}$ are cyclic, so as $U_{1}=U_{A} U_{B}$, for each $p \in \pi\left(U_{1}\right), U_{A}$ or $U_{B}$ contains a Sylow $p$-subgroup of $U_{1}$, 
and then $U_{A}=U_{B}=U_{1}$ by (3.2), contradicting $\alpha>1$. Finally, (3.2) is impossible in case (iv) from the factorizations of $G_{2}(q)$ listed in [LPS2, Theorem B].

Therefore $A$ and $B$ are maximal in $L$ and described in one of the four cases above. We next argue as in [LPS1, proof of 4.3] that case (iv) does not satisfy our hypothesis. Assume that (iv) holds. We saw that $U=U_{1} \cong G_{2}(q)$ and $A \cong \Omega_{7}(q)$. Suppose first that $v>2$. Then $A^{\prime} \leq B \cap D$ where $D$ is a third member of $A^{M}$. By symmetry, $B \cap D \cong G_{2}(q)$. But as $B \cap D \cong G_{2}(q)$, $|L|$ does not divide $|A||B \cap D|$ as $\left(q^{2}+1, q^{6}-1\right)=(q-1,2)$. Thus $|L|$ does not divide $|A|\left|A^{\prime}\right|$, and this contradicts the hypothesis in (3.1) that $L=A A^{\prime}$.

Therefore $v=2$, so $U$ is $M$-invariant, so that $U=M_{L}$ as $\left\{M_{L}\right\}=\mathcal{I}_{L}^{*}(M)$. Therefore $M=N_{G}(U)$ and $\left|G: L N_{G}(A)\right| \leq\left|G: L N_{M}(A)\right| \leq\left|M: N_{M}(A)\right|=2$. But then from $[\mathrm{K}], N_{G}(U)$ acts on an $\Omega_{7}(q)$-subgroup of $L$, contradicting $\left\{M_{L}\right\}=\mathcal{I}_{L}^{*}(M)$.

Therefore one of cases (i)-(iii) holds. In each of these cases, $|X: Y|=2$, so as $A^{m}=B \notin A^{L}$ for $m \in M-N_{M}(L)$, it follows that $v=2$. Then as above, $U=M_{L}$, so $M=N_{G}(U)$. We proved that $A=A_{1}$, so $A$ is described in (i)-(iii), and $U=U_{1}$ was described shortly thereafter. Finally, we can determine $G$, and hence also $M=N_{G}(U)$, by inspecting $\operatorname{Aut}(L)$, to complete the proof of the lemma.

LeMma 3.5. Assume that $G$ is a finite simple group. Then there exists $p \in \pi(G)$ such that $G$ has cyclic Sylow p-subgroups.

PROOF. This seems to be part of the folklore, but as I do not know of a reference, here is a sketch of a proof.

It suffices to exhibit a prime which divides $|G|$ to the first power, and it is often possible to establish this stronger statement. If $G$ is of prime order this is trivial, so we may assume that $G$ is nonabelian. If $G$ is sporadic, such a prime exists by inspection of the orders of the sporadic groups (see [GLS3]).

Suppose that $G \cong A_{n}$. By Bertrand's postulate (see [NZ, Theorem 8.6]) there is a prime $p$ such that $n / 2<p \leq n$. Then $p$ divides $|G|$ to the first power, so the lemma holds in this case too.

Thus we may assume that $G$ is of Lie type. Let $G_{0}$ be the universal group of Lie type of type $G$. From [GLS3, 4.10.1],

$$
\left|G_{0}\right|=q^{N} \prod_{i} \Phi_{i}(q)^{n_{i}}
$$

for suitable integers $N, n_{i}$ and prime power $q$, where $\Phi_{m}(x)$ is the $m$ th cyclotomic polynomial. Let $p$ be a prime relatively prime to $q$, and $m_{0}$ the order of $q$ in the multiplicative group of $\mathbf{F}_{p}$. By [GLS3, 4.10.3.a], $m_{p}\left(G_{0}\right)=n_{m_{0}}$. Thus it remains to pick $p$ with $n_{m_{0}}=1$.

Next by Zsigmondy's theorem (see [Gu, 3.2]), given $i$, there is a prime $p$ with $m_{0}=i$, unless $(q, i)=(2,6)$ or $q$ is a Mersenne prime and $i=2$. Thus it suffices to exhibit an $i$ with $n_{i}=1$ and $(q, i)$ not one of the exceptional Zsigmondy pairs. If $G$ is of type ${ }^{3} D_{4}(q)$, then the factorization (3.3) appears after [GLS3, 4.10.1], and by inspection $i=12$ works. In the remaining case we examine the standard factorization 


$$
\left|G_{0}\right|=q^{N} \prod_{j}\left(q^{d_{j}}-\epsilon_{j}\right)
$$

appearing for example in [GLS3, Table 2.2] or [A2, Table 16.1], where the $d_{j}$ are suitable positive integers and $\epsilon_{j} \in\{1,-1\}$. Now $\Phi_{u_{j} d_{j}}(q)$ divides $q^{d_{j}}-\epsilon_{j}$, for $u_{j}=1,2$ when $\epsilon_{j}=1,-1$, respectively, and we choose $i=u_{j} d_{j}$ with $d_{j}$ maximal. This choice works unless $(q, i)$ is an exceptional Zsigmondy pair. From the factorization, $n_{i}=1$, and $i=2$ if and only if $G=L_{2}(q)$, while $(q, i)=(2,6)$ if and only if $G$ is $L_{6}^{\epsilon}(2), S p_{6}(2)$, or $G_{2}(2)$. We choose $p=q, 31,11,7,7$ when $G$ is $L_{2}(q)$, $L_{6}(2), U_{6}(2), S p_{6}(2), G_{2}(2)$, respectively.

Recall (see [A2]) that for $G$ a finite group and $p$ a prime, $m_{p}(G)$ is the $p$-rank of $G$.

LEMMA 3.6. Assume that $\Omega$ is a finite set, $G \leq \operatorname{Sym}(\Omega)$, and $H$ is a subgroup of $G$ regular on $\Omega$. Let $p$ be a prime, $P \in \operatorname{Syl}_{p}(H)$, and $P \leq Q \in \operatorname{Syl}_{p}(G)$.

(1) There exists $\omega \in \Omega$ such that $Q_{\omega} \in \operatorname{Syl}_{p}\left(G_{\omega}\right)$, and for each such $\omega, P$ is a complement to $Q_{\omega}$ in $Q$.

(2) If $Q$ is abelian then $Q=P \times Q_{\omega}$ and $m_{p}(G)=m_{p}(H)+m_{p}\left(G_{\omega}\right)$.

PROOF. The first remark in (1) follows from Sylow's theorem. Next $|G|=|\Omega|\left|G_{\omega}\right|$, so

$$
|Q|=|G|_{p}=|\Omega|_{p}\left|G_{\omega}\right|_{p}=|P|\left|Q_{\omega}\right|,
$$

as $H$ is regular on $\Omega, P \in \operatorname{Syl}_{p}(H)$, and $Q_{\omega} \in \operatorname{Syl}_{p}\left(G_{\omega}\right)$. As $P$ is semiregular on $\Omega, P \cap Q_{\omega}=1$, and (1) follows from this observation together with (3.4). Then (1) implies (2).

LEMMA 3.7. Assume that $G$ is almost simple and set $L=F^{*}(G)$. Assume that $1 \neq H<L$ and $|G: L|$ is prime. Let $\mathcal{M}$ be the set of maximal overgroups of $H$ in $G$ and assume that,

$$
\text { for each } M \in \mathcal{M} \text {, there exists } M^{\prime} \in \mathcal{M}-\{M\} \text { with } H=M \cap M^{\prime} \text {. }
$$

Then:

(1) L and $N_{G}(H)$ are in $\mathcal{M}$;

(2) $H=N_{L}(H)$;

(3) $\left|N_{G}(H): H\right|=|G: L|$;

(4) for each $M \in \mathcal{M}-\left\{N_{G}(H)\right\}, H=M \cap N_{G}(H)$.

Proof. As $|G: L|$ is prime and $H \leq L, L \in \mathcal{M}$. Thus by (3.5), there exists $K \in \mathcal{M}-\{L\}$ with $H=L \cap K$. As $L \unlhd G, H \unlhd K$, so $K=N_{G}(H)$ by maximality of $K$. Thus (1) holds, and $H=L \cap K=N_{L}(H)$, establishing (2). As $|G: L|$ is prime and $K \in \mathcal{M}-\{L\},|G: L|=|K: K \cap L|=|K: H|$, so (3) holds. Finally, if $M \in \mathcal{M}-\{K\}$ then $M \cap K=N_{M}(H)=H$ or $K$ as $H \leq M \cap K$ and $|K: H|$ is prime. But as $M$ and $K$ are distinct maximal subgroups of $G, K \not \leq M$, so $M \cap K=H$, establishing (4). 
LEMMA 3.8. Assume that $G$ is almost simple and set $L=F^{*}(G)$. Assume that $|G: L|$ is prime and $H$ is a maximal subgroup of $L$ such that $N_{G}(H) \not L$. Let $\mathcal{M}$ be the set of maximal overgroups of $H$ in $G$.

(1) $\mathcal{M}=\left\{L, N_{G}(H)\right\}$.

(2) $\mathcal{O}_{G}(H)=\left\{H, L, N_{G}(H), G\right\}$.

Proof. As $|G: L|$ is prime and $H \leq L, L \in \mathcal{M}$. As $|G: L|$ is prime and $K=N_{G}(H) \not \leq L, G=L K$ and $|K: K \cap L|=|G: L|$. Let $J \in \mathcal{O}_{G}(H)-\{G, L\}$. Then as $H$ is maximal in $L, H=J \cap L \unlhd J$, so $J \leq K$. In particular, $H=K \cap L$, so $|K: H|$ is prime, and hence $J=H$ or $K$. The lemma follows.

\section{Overgroups of primitive groups}

In this section we assume Hypothesis 2.1. Recall the definition of the various types of primitive groups from Definition 2.3. Recall also that for $M$ a suitable primitive subgroup of $S, \mathcal{F}(M)$ is the $M$-invariant product structure on $\Omega$ defined in Notation 2.6. Similarly, for $H$ affine, $\mathcal{D}(H)$ is the set of $H$-invariant direct sum decompositions of $F^{*}(H)$ defined in Notation 2.6. Given a suitable collection $\mathcal{L}$ of subgroups of $S$, the product structure $\mathcal{F}(\mathcal{L})$ is defined in Example 1.6.

LEMMA 4.1. Assume that $H$ is affine and $M \in \mathcal{O}_{G}(H)$. Then $H$ preserves the affine structure $R=R(D)$ defined in Lemma 1.4, $D \leq F^{*}(M)$, and one of the following statements holds:

(1) $M$ is affine with $F^{*}(M)=D$, so $M \leq N_{G}(R)$.

(2) $H$ is imprimitive on $D$ and there exists $\mathcal{D}=\left\{D_{1}, \ldots, D_{k}\right\} \in \mathcal{D}(H)$ such that $M$ is semisimple and $\mathcal{F}(M)=\mathcal{F}(\mathcal{D})$. Moreover, $d=\left|D_{1}\right| \geq 5$, and $D_{i}=D \cap X_{i}$ is a complement to $X_{i, \omega}$ in $X_{i}$, where $\left\{X_{1}, \ldots, X_{k}\right\}$ are the components of $M$. Furthermore, if $M=N_{G}(\mathcal{F}(M))$ then $N_{M}(D)$ is the stabilizer in $N_{G}(D)$ of $\mathcal{D}$.

(3) $|\Omega|=p$ is prime.

PROOF. By Lemma 1.4, $H$ preserves $R$.

Let $X=F^{*}(M)$. By Lemma $2.4, M$ is primitive on $\Omega$, so $X$ is transitive on $\Omega$. In particular, $p \in \pi(X)$, so $1 \neq C_{X}(D)$. But as $D$ is regular on $\Omega, D=C_{S}(D)$, so $1 \neq D \cap X$, and hence $D \leq X$ by Lemma 2.4(2). As $D$ is regular on $\Omega, D$ is a complement to $X_{\omega}$ in $X$.

Next $M$ is described in Lemma 2.2, so as $|\Omega|=p^{e}$ is a power of a prime, we conclude (using Lemma 1.4 in case (3i) of Lemma 2.2) that one of the following statements holds:

(i) $\quad M$ is affine;

(ii) $\quad M$ is semisimple and stabilizes the $(d, k)$-product structure $\mathcal{F}=\mathcal{F}(M)=\mathcal{F}(\mathcal{L})$, where $\mathcal{L}=\left(X_{i}: 1 \leq i \leq k\right)$ is the set of components of $M, d=p^{f}$ for some divisor $f$ of $e$, and $k=e / f>1$;

(iii) $M$ is almost simple. 
In case (i), $|X|=|\Omega|=|D|$, so $X=D$ and hence (1) holds. Thus we may assume that case (ii) or (iii) holds. Furthermore, we may assume (3) does not hold, so $e>1$.

Suppose that case (iii) holds. Recall that $D$ is an abelian complement to $X_{\omega}$ in $X$. Thus as $e>1$, Lemma 3.2 says that case (a) of Lemma 3.1 holds. This is a contradiction as $F^{*}(S) \not M$.

Thus we may assume that case (ii) holds. Let $D_{i}$ be the projection of $D$ on $X_{i}$. Then $D_{i} \leq C_{S}(D)=D$, so $D=\prod_{i} D_{i}$ and $\mathcal{D}=\left\{D_{1}, \ldots, D_{k}\right\} \in \mathcal{D}(H)$. As $X=X_{\omega} D$ and $X_{i, \omega}$ is the projection of $X_{\omega}$ on $X_{i}$, it follows that $X_{i}=X_{i \omega} D_{i}$. Then as $D$ is regular on $\Omega, D_{i}$ is a complement to $X_{i, \omega}$ in $X_{i}$. Hence, $\mathcal{F}(\mathcal{D})=\mathcal{F}(M)$ by Lemma 1.7. Therefore, (2) holds in this case, and the proof of the lemma is complete.

Definition 4.2. Define a semisimple group $H$ to be octal if for each component $L$ of $H$, and for each $\omega \in \Omega, L \cong \operatorname{Aut}_{H}(L) \cong L_{3}(2)$ and $|\omega L|=8$.

Lemma 4.3. Let $M \in \mathcal{O}_{G}(H)$. Then either

(1) $D \leq F^{*}(M)$; or

(2) $H$ is semisimple and octal with $r$ components $\left\{E_{i} \mid 1 \leq i \leq r\right\}, E_{i} \cong L_{3}(2)$, $|\Omega|=8^{r}$, and $M$ is affine with

$$
X=F^{*}(M)=\prod_{i=1}^{r} X_{i}
$$

where $X_{i}=\left[X, E_{i}\right] \cong E_{8} . \quad$ In particular, $H X$ is affine, $\mathcal{D}=\left\{X_{1}, \ldots, X_{r}\right\}$ $\in \mathcal{D}(H X)$, and $\mathcal{F}(H)=\mathcal{F}(\mathcal{D})$.

Proof. Assume that $M$ is a counterexample, and let $X$ be a minimal normal subgroup of $M$. By Lemma 4.1, $H$ is not affine. By Lemma 2.4(2), either $D \cap X=1$ or $H$ is doubled and we may assume that $D_{1} \cap X=1$. Set $E=D_{1}$ if $H$ is doubled and $E=D$ otherwise. Thus $E \cap X=E \cap F^{*}(M)=1$.

Suppose first that $M$ is affine. Then $n=|\Omega|=|X|=p^{e}$, so from Lemma 2.2, $H$ is semisimple, and hence $D$ is the direct product of $r$ simple groups $E_{i}$ permuted transitively by $H$ and $\left|E_{i}: E_{i, \omega}\right|=p^{f}$, with $r f=e$. By Lemma 3.2, one of the following statements holds:

(i) for each $i$ there exists an abelian complement $Q_{i}$ to $E_{i, \omega}$ in $E_{i}$;

(ii) $p=2$ and $E_{i} \cong L_{2}(q)$, where $q=2^{f}-1$ is a Mersenne prime;

(iii) $p=3, f=3$, and $E_{i} \cong P S p_{4}(3)$.

Assume first that (i) holds, and let $Q=Q_{1} \cdots Q_{r}$. Then $Q$ is abelian and regular on $\Omega$, so $C_{S}(Q)=Q$. This is a contradiction as $C_{X}(Q) \neq 1=D \cap X$.

Next assume that (ii) or (iii) holds. As $D$ is transitive on $\Omega, D$ is not contained in the complement $M_{\omega}$ to $X$ in $M$, so $H^{1}(H, X) \neq 0$. On the other hand, $H \cong K=$ $H X \cap M_{\omega}$, and $H X$ is primitive on $\Omega$ by Lemma 2.4, so $K$ is irreducible on $X$. Therefore $H$ is irreducible on $X$, so by [AS, Theorem 3], 


$$
X=\bigoplus_{i=1}^{r} X_{i}
$$

where $X_{i}=\left[X, E_{i}\right]$. Let $d=\operatorname{dim}\left(\left[X, E_{i}\right]\right)$. Then $r d=e=r f$, so $d=f$. But $\left|P S p_{4}(3)\right|$ does not divide $\left|L_{3}(3)\right|$, so (iii) does not hold. Furthermore, in (ii), if $f>3$ and $P \in \operatorname{Syl}_{q}\left(E_{i}\right)$ then $N_{G L_{f}(2)}(P)$ is $P$ extended by a group of order $f$, whereas $\left|N_{E_{i}}(P)\right|=q(q-1) / 2$, which is a contradiction. Thus, in case (ii), $f=3$ and $E_{i} \cong L_{3}(2)$, so that (2) holds.

Thus we may assume that $M$ is not affine. If $M$ is almost simple then (1) holds by the Schreier property (see [GLS3, 7.1.1]). Therefore, as $M$ appears in case (2) or (3) of Lemma 2.2, $X$ is the direct product of $k$ simple components $X_{i}$, permuted transitively by $M$, and one of the following statements holds:

(a) $\quad M$ is semisimple and $n=m^{k}$, where $\left|X_{i}: X_{i, \omega}\right|=m$;

(b) $M$ is doubled or complemented, and $n=y^{k}$, where $\left|X_{i}\right|=y$;

(c) $\quad M$ is diagonal and $n=y^{k-s}$, where $\left|X_{i}\right|=y$ and $s=|\Sigma(M)|$.

Next $F^{*}(M) \leq U \unlhd M$ with $U / F^{*}(M)$ solvable and $M / U \leq S_{k}$. Therefore, as $E$ is the direct product of simple components and $E \cap F^{*}(M)=1$, we conclude that $E \cap U=1$, so $E$ is isomorphic to a subgroup of $S_{k}$. However, $E$ is transitive on $\Omega$, so $|E|$ is divisible by $n=m^{k}, y^{k}$, or $y^{k-s}$, in (a), (b), or (c), respectively. Pick $p$ to be a prime divisor of $m$ or $y$ in the respective case. We may pick $p$ to be odd, except possibly in case (a). Thus $a=\log _{p}\left(|E|_{p}\right) \geq k$ in (a) and (b), while $a \geq k-s$ in (c). In particular, $a \geq k$ if $p=2$. But by Lemma 3.3, $l=\log _{p}\left((k !)_{p}\right) \leq(k-1) /(p-1)$, while if $p>2$ then $(k-1) /(p-1) \leq(k-1) / 2<k-s$. Thus in any event, $l<a$, in contradiction to $E$ being isomorphic to a subgroup of $S_{k}$.

LEMMA 4.4. Let $M \in \mathcal{O}_{G}(H)$ be almost simple. Then one of the following statements holds:

(1) $\quad M=S$ or $F^{*}(S)$;

(2) $H$ is almost simple;

(3) $H$ is affine and $\Omega$ is prime.

Proof. Assume that the lemma is false and pick a pair $\mathcal{P}=(M, H)$ which is a counterexample to the lemma, and with $|M: H|$ minimal subject to this constraint. By Lemma $4.1, H$ is not affine, and as $\mathcal{P}$ is a counterexample, $H$ is not almost simple. Therefore $H$ is doubled, complemented, or semisimple. Let $X=F^{*}(M)$. As $H X$ satisfies the hypothesis of the lemma, $M=H X$ by minimality of $\mathcal{P}$. By Lemma 4.3, $D \leq X$.

Let $K=M_{\omega}$ and $J=K \cap X$. As $D$ is transitive on $\Omega, M=K D$. Then as $D \leq X$, also $X=J D$. By Lemma 2.4, $M$ is primitive on $\Omega$, so $K$ is maximal in $M$.

Suppose that $H$ is not maximal in $M$, and let $I \in \mathcal{O}_{M}(H)$ with $H$ maximal in $I$. By Lemma $2.4, I$ is primitive on $\Omega$. By minimality of $\mathcal{P}, I$ is not almost simple. If $|\Omega|$ is a prime, then $H$ is affine or almost simple by Lemma 2.2, contrary to an earlier 
observation. Thus $M=S$ or $F^{*}(S)$ by minimality of $\mathcal{P}$, contrary to the choice of $\mathcal{P}$ as a counterexample.

Therefore $H$ and $K$ are maximal in $M$, so $M=K H$ is a maximal factorization of $M$ with the following properties:

(a) $\quad X=(X \cap K)(X \cap H)$ is also a factorization;

(b) $H$ is primitive on $\Omega$ so $K \cap H=H_{\omega}$ is maximal in $H$;

(c) $D=F^{*}(H)$ is the direct product of $r^{\prime}>1$ isomorphic nonabelian simple groups.

To complete the proof of the lemma, we inspect the list of maximal factorizations of the almost simple groups in [LPS2], and verify that no pair on the lists satisfies (a)-(c).

We begin with the case where $X$ is a classical group. Thus we appeal to [LPS2, Theorem A], and inspect [LPS2, Tables 1-4]. We first search for pairs satisfying (c). In Table 1, the only examples occur with $X=S p_{2 m}(q), q$ even. The last four rows of that subtable contain a $(*)$, so they do not satisfy (a). The remaining pairs $(A, B)$ satisfying (c) are $\left(S p_{2}\left(q^{2}\right) .2, O_{4}^{+}(q)\right)$ and $\left(O_{2 m}^{-}(q), S p_{m}(q)\right.$ wr $\left.\mathbf{Z}_{2}\right)$ with $m$ even. Both pairs fail to satisfy (b): see the discussion in [LPS2, 3.2.1.d p. 48, and 3.2.4.b p. 50, respectively].

In Table 2, the only pair satisfying (c) is $\left(S z(q), O_{4}^{+}(q)\right)$ in $\operatorname{PSp}_{4}(q)$. However, here $A \cap B$ is a dihedral group of order $2(q-1)$, which is not maximal in $H$, so (b) fails.

There are no examples of pairs in Table 3 satisfying (c). In Table 4 there are two examples: $\left(\Omega_{7}(2),\left(L_{2}(4) \times L_{2}(4)\right) .2^{2}\right)$ in $\Omega_{8}^{+}(2)$, and an example for $\Omega_{8}^{+}(4)$ which fails (a) as a $(*)$ appears in the corresponding row. Consider the first example. Here (see the discussion in [LPS2, 3.6.1.c]) $X$ is $\Omega_{8}^{+}(2)$, and if we write $V$ for the orthogonal space defining $X$, then, up to conjugation in $\operatorname{Aut}(X), A$ is the stabilizer of a nonsingular vector $v \in V$, and $B$ is the stabilizer of an extension field structure over $\mathbf{F}_{4}$, and isomorphic to $O_{4}^{+}(4)$ extended by a field automorphism. Now $A \cap B=$ $C_{B}(v) \cong \mathbf{Z}_{2} \times O_{3}(4)$ is not maximal in $B$, giving a contradiction. This completes the analysis in the case when $X$ is a classical group.

When $X$ is an exceptional group of Lie type, we see from [LPS2, Theorem B and Table 5] that there are no examples of pairs satisfying (c). Similarly, from [LPS2, Theorem $\mathrm{C}$ and Table 6], when $X$ is sporadic there are no examples satisfying (c).

Finally, assume that $X$ is an alternating group $A_{n}$ with $n=7$ or $n>8$. We appeal to [LPS2, Theorem D]. We first consider the generic examples in that theorem, where $A$ is the stabilizer of a $k$-subset $\Gamma$ in the $n$-set $\Sigma$ permuted by $M$, with $1 \leq k \leq 5$, and $B$ is $k$-homogeneous on $\Sigma$. As $\mathcal{P}$ does not satisfy conclusion (1) of our lemma, $k>1$. As $B$ is $k$-homogenous, either $B$ is 2-transitive or $k=2$ and $B$ is of odd order. In either case $B$ does not satisfy (c), so $A$ satisfies (c). Thus $k=5$ and $n=10$. But as $B<M$ is 5-homogeneous, $n=12$ or 24 and $B$ is $M_{12}$ or $M_{24}$, giving a contradiction.

This leaves the exceptional cases in Theorem $\mathrm{D}$, and as $n$ is not 6 or 8 , we have $n=10$. The only example satisfying (c) has $A$ equal to $L_{2}(8)$ or ${ }^{2} G_{2}(3)$, and $D \cong A_{5} \times A_{5}$. From the discussion on [LPS2, p. 124], $A \cap B \cong A_{4}$, so $A \cap B$ is not maximal in $B$, and hence (b) is not satisfied. Thus the proof is complete at last. 
LEMMA 4.5. If $M \in \mathcal{O}_{G}(H)$ is complemented then $H$ is complemented and $F^{*}(M)$ $=F^{*}(H)$.

Proof. By Lemma 4.3, $D \leq F^{*}(M)=X$. As $M$ is complemented, $X$ is regular on $\Omega$, so as $D$ is transitive on $\Omega, D$ is also regular on $\Omega$. In particular, $|D|=|\Omega|=|X|$, so $D=X$. As $M$ is complemented, $|\Omega|$ is not a prime power. But from Lemma 2.2, as $D$ is regular on $\Omega$, either $H$ is affine and $|\Omega|$ is a prime power, or $H$ is complemented. Therefore $H$ is complemented.

Lemma 4.6. Assume that $H$ is complemented and let $D_{2}=C_{G}(D)$.

(1) The map $\varphi: g \mapsto \omega g$ is an equivalence of the representation $\rho$ of $D$ by right multiplication on $D$ with the representation of $D$ on $\Omega$.

(2) Define $\lambda: D \rightarrow \operatorname{Sym}(D)$ by $x \lambda: g \mapsto x^{-1} g$, and let $\psi=\lambda \varphi^{*}$, where $\varphi^{*}$ : $\operatorname{Sym}(D) \rightarrow S$ is defined by $\varphi^{*}: \beta \mapsto \varphi^{-1} \beta \varphi$. Then $\psi: D \rightarrow S$ is a permutation representation with $D \psi=D_{2}$.

(3) Let $X=D D_{2}$ and $F=\{g \cdot g \psi \mid g \in D\}$. Then $F=X_{\omega}$ is an $H_{\omega}$-invariant full diagonal subgroup of $X$.

(4) $H_{G}(D)=X H \in \mathcal{O}_{G}(H)$ is doubled.

PROOF. Part (1) is a restatement of the fact in Lemma 2.2 that $D$ is regular on $\Omega$.

Visibly $\lambda$ and $\psi$ are permutation representations with $D \psi$ a subgroup of $D_{2}$ regular on $\Omega$. As $D$ is transitive on $\Omega, D_{2}$ is semiregular on $\Omega$, so as $D \psi$ is a transitive subgroup of $D_{2},(2)$ holds.

For $g \in D$, let $g \alpha=g \cdot g \psi$. Observe that $1(g \rho \cdot g \lambda)=g^{-1} g=1$, so $g \rho \cdot g \lambda$ fixes 1 , and hence $g \alpha=g \rho \varphi^{*} \cdot g \lambda \varphi^{*} \in X_{\omega}$. As $D$ is transitive on $\Omega,\left|X_{\omega}\right|=|X| /|\Omega|=|F|$, so $F=X_{\omega}$. As $H_{\omega}$ acts on $D$, it also acts on $X=D C_{G}(D)$, and then on $X_{\omega}=F$, completing the proof of (3).

As $H$ acts on $C_{G}(D), H C_{G}(D)=H X$ is a subgroup of $G$, so $X H \in \mathcal{O}_{G}(H)$. Then $X H$ is primitive on $\Omega$ by Lemma 2.4, and from (3), $H X$ is doubled.

In the next lemma, $\operatorname{diag}(\mathcal{B}, F)$ is defined in Definition 1.9 , while $\mathcal{P}(H)$ and $\mathcal{F}(H . \Delta)$ are defined in Notation 2.6.

LeMma 4.7. Assume that $H$ is doubled with minimal normal subgroups $D_{1}$ and $D_{2}$, and let $\mathcal{L}$ be the set of components of $D_{1}$. Let $k=|\mathcal{L}|$ and $m=|L|$ for $L \in \mathcal{L}$.

(1) If $k>1$, then for each $\Delta \in \mathcal{P}(H), \mathcal{F}(H, \Delta)$ is a regular $\left(m^{k / d}, d\right)$-product structure preserved by $H$, where $d=|\Delta|$. In particular, $\mathcal{F}(H)$ is an $(m, k)$ structure.

(2) If $k=1$, set $\mathcal{B}=\left\{D_{1}, D_{2}\right\}$ and $F=D_{\omega}$. Then $\mathbf{d}(H)=\operatorname{diag}(\mathcal{B}, F)$ is an $H$ invariant diagonal structure on $\Omega$.

PROOF. Part (1) follows from Notation 2.6. On the other hand, if $k=1$ then (2) follows from the definition of a diagonal structure in Definition 1.9. 
LEMMA 4.8. Assume that $M \in \mathcal{O}_{G}(H)$.

(1) If $M$ is doubled or diagonal then $H$ is not semisimple.

(2) If $M$ is doubled then $H$ is not diagonal.

(3) If $M$ and $H$ are doubled then $F^{*}(H)=F^{*}(M)$.

Proof. Assume the hypothesis of one of (1)-(3), and let $X=F^{*}(M)$. By Lemma $4.3, D \leq X$, so $D_{\omega} \leq X_{\omega}$.

First assume the hypothesis of (3). Then $|X|=|\Omega|^{2}=|D|$, so $D=X$ and hence (3) holds.

Now assume that $M, H$ is a counterexample to (1) or (2). If $H$ is semisimple let $\mathcal{L}$ be the set of components of $H$, while if $H$ is diagonal let $\mathcal{L}=\left\{D_{\sigma} \mid \sigma \in \Sigma(H)\right\}$ (in the notation of Lemma 2.2). If $M$ is doubled let $J$ be one of the minimal normal subgroups of $M$, while if $M$ is diagonal, then for $\gamma \in \Sigma(M)$, let $J_{\gamma}$ be the product of $|\gamma|-1$ of the components of $X_{\gamma}$, and set $J=\left\langle J_{\gamma}: \gamma \in \Sigma(M)\right\rangle$.

In either case, $J$ is regular on $\Omega$ and we can write $X=J \times J^{\prime}$, where $J^{\prime}$ is the product of the components of $M$ not contained in $J$. Let $\pi: X \rightarrow J$ be the projection map with respect to this direct sum decomposition. Let $\alpha=\pi_{\mid X_{\omega}}: X_{\omega} \rightarrow J$, and observe that $\alpha$ is an injection as $J^{\prime}$ is semiregular on $\Omega$. Thus, $\alpha: L_{\omega} \rightarrow J$ is injective for each $L \in \mathcal{L}$. Therefore as $L$ is a minimal normal subgroup of $N_{H}(L) \cap N_{H}(J)$, and as $L_{\omega} \neq 1$, it follows that $\pi: L \rightarrow J$ is injective. Then as each nontrivial normal subgroup of $D$ intersects some $L \in \mathcal{L}$ nontrivial, it follows that $\pi: D \rightarrow J$ is an injection. But now $|\Omega|<|D| \leq|J|=|\Omega|$, giving a contradiction.

\section{Semisimple overgroups of primitive groups}

In this section we make the following assumption.

Hypothesis 5.1. Hypothesis 2.1 is satisfied and $H$ is not affine. Furthermore, $M \in \mathcal{O}_{G}(H)$ and $M$ is semisimple but not almost simple.

The referee pointed out that [BPS2] contains results similar to those in this section, and indicates that [BPS2] can be used to prove many of the lemmas in the section.

Notation 5.2. Let $X=F^{*}(M), I=\{1, \ldots, k\}$, and $\bar{I}=\{1, \ldots, r\}$. Pick $\omega \in \Omega$.

Let $\mathcal{X}=\left\{X_{i} \mid i \in I\right\}$ be the set of components of $M$. For $\gamma \subseteq I$, set $\gamma^{\prime}=I-\gamma$, $X_{\gamma}=\left\langle X_{i}: i \in \gamma\right\rangle, \Gamma_{\gamma}=\omega X_{\gamma}$, and $\pi_{\gamma}: X \rightarrow X_{\gamma}$ the projection map with respect to the direct sum decomposition $X=X_{\gamma} \times X_{\gamma^{\prime}}$.

As $M$ is semisimple but not almost simple, $k>1$ and $M$ preserves the $(m, k)$ product structure $\mathcal{F}=\mathcal{F}(M)=\left(\Omega_{i}: i \in I\right)$ defined in Notation 2.6, with $X_{i}$ transitive on $\Omega_{i}$ and $X_{i^{\prime}}$ the kernel of the action of $X$ on $\Omega_{i}$. This allows us to identify $\Omega$ with $\prod_{i \in I} \Gamma_{i}$, as in the discussion in Definition 1.5.

Let $E$ be a minimal normal subgroup of $H$ and $\mathcal{E}=\left\{E_{i} \mid i \in \bar{I}\right\}$ the set of components of $E$. By Lemma 4.3, $E \leq X$. For $i \in \bar{I}$ let

$$
\beta_{i}=\left\{j \in I \mid E_{i} \pi_{j} \neq 1\right\}
$$


and for $i \in I$ let

$$
S_{i}=\left\{j \in \bar{I} \mid E_{j} \pi_{i} \neq 1\right\} .
$$

Represent $H$ on $I$ and $\bar{I}$ so that the maps $i \mapsto X_{i}$ and $j \mapsto E_{j}$ are equivalences of permutation representations. As $H$ is transitive on $\mathcal{E}$ and acts on $\mathcal{X}, H$ is transitive on $\bar{I}$ and

$$
b=b(M, H)=\left|\beta_{i}\right|
$$

is independent of $i \in \bar{I}$. For $\gamma \subseteq I$ define

$$
Q_{\gamma}=\left\{e \in E \mid e \pi_{\gamma} \in X_{\gamma, \omega}\right\} \text {. }
$$

See Notation 2.6 for the definition of $\mathcal{F}(H, K)$ for suitable $K \leq H$.

LEMMA 5.3. (1) For each $\gamma \subseteq I, E=Q_{\gamma} Q_{\gamma^{\prime}}$.

(2) For each proper nonempty subset $\gamma$ of $I, E_{\omega}<Q_{\gamma}<E$, and $E \pi_{\gamma}$ is transitive on $\Gamma_{\gamma}$.

(3) If $i, j \in I$ are distinct, then $E=Q_{i} Q_{j}$ and $Q_{j} \notin Q_{i}^{E}$.

(4) $H$ is transitive on $\mathcal{X}$ and $I$.

Proof. Let $\gamma \subseteq I$ and $x \in X_{\gamma}$. As $E$ is transitive on $\Omega, x=e v$ for some $e \in E$ and $v \in X_{\omega}$. As $M$ is semisimple, $X_{\omega}=X_{\gamma, \omega} \times X_{\gamma^{\prime}, \omega}$, so $v \pi_{\alpha} \in X_{\alpha, \omega}$ for $\alpha \in\left\{\gamma, \gamma^{\prime}\right\}$. Thus $E_{\omega} \leq Q_{\gamma}$ and

$$
e \pi_{\gamma^{\prime}}=\left(x v^{-1}\right) \pi_{\gamma^{\prime}}=\left(v^{-1}\right) \pi_{\gamma^{\prime}} \in X_{\gamma^{\prime}, \omega},
$$

so $e \in Q_{\gamma^{\prime}}$. By symmetry, for $x^{\prime} \in X_{\gamma^{\prime}}, x^{\prime}=e^{\prime} v^{\prime}$ with $e^{\prime} \in Q_{\gamma}$ and $v^{\prime} \in X_{\omega}$. Next for each $g \in E, g=x x^{\prime}$ with $x \in X_{\gamma}$ and $x^{\prime} \in X_{\gamma^{\prime}}$, so $g=e v e^{\prime} v^{\prime}$ with $e \in Q_{\gamma^{\prime}}$ and $v e^{\prime} v^{\prime} \in Q_{\gamma}$. This establishes (1).

As $X=X_{\omega} E, X_{\gamma}=X \pi_{\gamma}=X_{\omega} \pi_{\gamma} E \pi_{\gamma}=X_{\gamma, \omega} E \pi_{\gamma}$, so $E \pi_{\gamma}$ is transitive on $\Gamma_{\gamma}$, and hence if $\gamma \neq \varnothing$ then $E \pi_{\gamma} \not X_{\gamma, \omega}$, so that $Q_{\gamma} \neq E$. Similarly, by (1), $E \pi_{\gamma^{\prime}} \leq$ $Q_{\gamma} \pi_{\gamma^{\prime}} X_{\gamma^{\prime}, \omega}$ and if $\gamma^{\prime} \neq \varnothing$ then $X_{\gamma^{\prime}, \omega} \neq X_{\gamma^{\prime}}=E \pi_{\gamma^{\prime}} X_{\gamma^{\prime}, \omega}$, so $Q_{\gamma} \neq E_{\omega}$. Therefore (2) holds.

Let $\gamma$ be an orbit of $H$ on $I$. Then $H_{\omega}$ acts on $Q_{\gamma}$, and if $\gamma \neq I$ then by (2), $H_{\omega}<H_{\omega} Q_{\gamma}<H$, in contradiction to $H$ being primitive on $\Omega$. Thus (4) holds.

Finally, suppose that $i, j \in I$ are distinct. Then $j \in i^{\prime}$, so $Q_{i^{\prime}} \leq Q_{j}$, and hence $E=Q_{i} Q_{j}$ by (1). Therefore $Q_{j}$ is transitive on $E / Q_{i}$, and by (2), $\left|E / Q_{i}\right|>1$, so that $Q_{j}$ fixes no point of $E / Q_{i}$. Hence $Q_{j} \notin Q_{i}^{E}$, establishing (3).

LEMMA 5.4. (1) $s=\left|S_{i}\right|$ is independent of $i \in I$.

(2) $k s=r b$.

(3) $\quad b=1$ if and only if each component of $E$ is contained in a component of $X$.

(4) $|\Omega|=m^{k}$.

Proof. Part (1) follows from Lemma 5.3(4). Then (2) follows from counting the order of $\left\{(i, j) \in I \times \bar{I} \mid E_{j} \pi_{i} \neq 1\right\}$ in two ways. Part (3) is trivial, and (4) follows from Lemma 2.2. 
LeMma 5.5. Assume that $b=1$. Then the following statements hold.

(1) $r=k s$.

(2) For $i \in I, S_{i}=\left\{j \in \bar{I} \mid E_{j} \leq X_{i}\right\}$.

(3) $\mathcal{S}=\left\{S_{i} \mid i \in I\right\}$ is an $H$-invariant partition of $\bar{I}$ into $k$ blocks of size $s$.

(4) For $i \in I$, let $E(i)=\left\langle E_{j} \mid j \in S_{i}\right\rangle$ and $H_{i}$ be the stabilizer in $H$ of $i$. Then $E(i)=E \pi_{i}$ is transitive on $\Gamma_{i}, H_{i}$ is transitive on $S_{i}$, and $H_{i}$ is primitive on $\Gamma_{i}$.

(5) Either $H$ is semisimple and $s=1$, or $X_{i} \cong A_{m}$ is the alternating group on $\Gamma_{i}$.

(6) $\mathcal{F}=\mathcal{F}\left(H, H_{1}\right)$.

(7) If $H$ is doubled or semisimple, then $\operatorname{Aut}_{H}\left(X_{1}\right)$ is doubled or semisimple on $\Gamma_{1}$, respectively.

(8) If $H$ is diagonal then $\Sigma(H)$ corresponds to an $H$-invariant partition $\Sigma$ of $\bar{I}$ under the equivalence of Notation 5.2, the partition $\Sigma$ is a refinement of $\mathcal{S}$, and $\operatorname{Aut}_{H}\left(X_{1}\right)$ is diagonal on $\Gamma_{1}$.

(9) If $H$ is complemented then Aut $_{H}\left(X_{1}\right)$ is complemented or doubled on $\Gamma_{1}$.

PROOF. Part (1) follows from Lemma 5.4(2), while (2) follows from Lemma 5.4(3). Then (2) implies (3).

Let $i \in I$. By (2), $E(i)=E \pi_{i}$, so $E(i)$ is transitive on $\Gamma_{i}$ by Lemma 5.3(2). By (3) and Lemma 5.3(4), $H_{i}$ is transitive on $S_{i}$ and for $j \in S_{i}, N_{H}\left(E_{j}\right) \leq H_{i}$.

If $H$ is semisimple then by Lemma 2.2, $E_{\omega}$ is the direct product of the groups $E_{j, \omega}, j \in \bar{I}$, and $\operatorname{Aut}_{H_{\omega}}\left(E_{j}\right)$ is maximal in $\operatorname{Aut}_{H}\left(E_{j}\right)$. Thus as $N_{H}\left(E_{j}\right) \leq H_{i}$, as $H_{i}$ is transitive on $S_{i}$, and as $E(i)$ is transitive on $\Gamma_{i}, H_{i}$ is primitive and semisimple on $\Gamma_{i}$. Therefore in this case, (4) and (7) hold, while (5) follows from Lemma 4.4 and (6) follows from Notation 2.6.

If $H$ is doubled then by Lemma 2.2, D=E $\times \tilde{E}$ with $D_{\omega}$ a full diagonal subgroup of $D$. As $E(i)$ is transitive on $\Gamma_{i}$ and semiregular on $\Omega, E(i)$ is regular on $\Gamma_{i}$. From part (1) of the next lemma (whose proof does not depend upon this lemma), $b(M, \tilde{E})=1$, so by symmetry between $E$ and $\tilde{E}, \tilde{E} \pi_{i}=\tilde{E} \cap X_{i}=\tilde{E}(i)$ is regular on $\Gamma_{i}$, and it the direct product of $\tilde{s}$ components. By (1), $\tilde{s}=s$. Thus $Y_{\omega}$ is a full diagonal subgroup of $Y=E(i) \tilde{E}(i)$. Hence $Y$ is primitive on $\Gamma_{i}$, and as $\operatorname{Aut}_{H}\left(X_{i}\right)$ has two distinct minimal normal subgroups, the group is doubled. Thus (4) and (7) hold in this case, and, as above, (5) and (6) follow from Lemma 4.4 and Notation 2.6.

Assume that $H$ is diagonal. Then the first statement in (8) follows from Lemma 2.2, which also says that for $\sigma \in \Sigma$, the global stabilizer $H(\sigma)$ in $H$ of $\sigma$ is primitive on $\sigma$. Let $1 \in S_{i} \cap \sigma$. As $H(\sigma)$ is primitive on $\sigma$, either $\sigma \subseteq S_{i}$ or $\{1\}=S_{i} \cap \sigma$. In the first case (8) holds, and (5) and (6) follow from Lemma 4.4 and Notation 2.6. Suppose that the second case holds. Then $E(i)$ is regular on $\Gamma_{i}$, so $m=\left|\Gamma_{i}\right|=|E(i)|=e^{s}$, where $e=\left|E_{1}\right|$. Thus appealing to Lemmas 2.2, 5.4(4), and (1),

$$
e^{r-|\Sigma|}=|\Omega|=m^{k}=e^{k s}=e^{r},
$$

giving a contradiction.

Finally, assume that $H$ is complemented. Then $E$ is regular on $\Omega$, so $E(i)$ is regular on $\Gamma_{i}$. If $H_{i, \omega}$ acts on some nontrivial proper subgroup $F$ of $E(i)$, then 
$H_{\omega}<\left\langle F^{H_{\omega}}\right\rangle H_{\omega}<H$, contradicting the maximality of $H_{\omega}$ in $H$. Therefore no such $F$ exists, so $H_{i, \omega}$ is maximal in $H_{i}$, and hence $H_{i}$ is primitive on $\Gamma_{i}$. As $E(i)$ is regular on $\Gamma_{i}, \operatorname{Aut}_{H}\left(X_{i}\right)$ is complemented or doubled. Thus (4) and (9) hold in this case, and (5) and (6) follow as usual.

LEMMA 5.6. Assume that $H$ is doubled or diagonal.

(1) $b=1$.

(2) $\quad X=F^{*}\left(M^{\prime}\right)$ where $M^{\prime}=N_{G}(X)=N_{G}(\mathcal{F})$, and $\mathcal{F}=\mathcal{F}\left(H, H_{1}\right)$, where $H_{1}=N_{H}\left(X_{1}\right)$.

Proof. First assume that $b>1$ and let $Y=X_{1^{\prime}}$ and $\Gamma=\Gamma_{1^{\prime}}$. Then $E \cap X_{1}=1$ by Lemma 5.4(3), so $\pi=\pi_{1^{\prime}}: E \rightarrow Y$ is an injection. Let $F=E \pi$ and for $i \in \bar{I}$, let $F_{i}=E_{i} \pi$. By Lemma 5.3(2) and the injectivity of $\pi, E_{\omega} \pi<Q_{1^{\prime}} \pi=P=F_{\omega}<F$, so there exists $a \in P-E_{\omega} \pi$. Let $a=a_{1} \cdots a_{u}$ with $a_{i} \in F_{i}^{\#}$, and $A=F_{1} \cdots F_{u}$.

Suppose first that $H$ is doubled. Then $D=E \times \tilde{E}$ and $D_{\omega}$ is a full diagonal subgroup of $D$. Then $B=D \pi=F \tilde{E} \pi$ and $R=D_{\omega} \pi \leq B_{\omega}$, so $R$ acts on $P=B_{\omega} \cap F$. Furthermore, $\operatorname{Aut}_{R}(A)=A$, so as $a_{i} \in F_{i}^{\#}$ and $F_{i}$ is simple, $A=$ $[a, R] \leq P$. Then as $A \unlhd F$ and $F$ is transitive on $\Gamma$ by Lemma 5.3(2), it follows that $A$ fixes $\Gamma$ pointwise, in contradiction to $Y$ being faithful on $\Gamma$.

Hence $H$ is diagonal. Then $F_{i}$ is contained in a block $\alpha_{i}$ of the partition of $\mathcal{E}$ in Lemma 2.2(3ii), and there is a full diagonal subgroup $U_{i}$ of $F_{\alpha_{i}}=\left\langle K: K \in \alpha_{i}\right\rangle$ contained in $F_{\omega}$. Then as above, $A=\left[a, U_{1} \cdots U_{u}\right] \leq P$, and we obtain the same contradiction. This completes the proof of (1).

By (1) and Lemma 5.5(5), $X_{1}$ acts as the alternating group on $\Gamma_{1}$. Thus $M^{\prime}=$ $N_{G}(X)=N_{G}(\mathcal{F})$ by Lemma 1.8. Then (2) follows from Lemma 5.5(6).

LEMMA 5.7. Let $\gamma \subseteq I$ and $\mu \subseteq \bar{I}$. For $\eta \subseteq \bar{I}$, let $E_{\eta}=\left\langle E_{i}: i \in \eta\right\rangle$ and $\eta^{\prime}=\bar{I}-\eta$. Let $\sigma: E \rightarrow E_{\mu}$ be the projection map with respect to the direct sum decomposition $E=E_{\mu} \times E_{\mu^{\prime}}$.

(1) If $Q_{\gamma} \sigma=E_{\mu}$ then $E_{\mu^{\prime}} \pi_{\gamma}$ is transitive on $\Gamma_{\gamma}$ and $E_{\mu} \pi_{\gamma}$ is semiregular on $\Gamma_{\gamma}$.

(2) Assume that $H$ is semisimple or complemented, $\mu=\{i\}$ for some $i \in \bar{I}$, and $\gamma$ is $N_{H_{\omega}}\left(E_{i}\right)$-invariant. Then for each $\alpha \in\left\{\gamma, \gamma^{\prime}\right\}, Q_{\alpha} \sigma \in\left\{E_{i, \omega}, E_{i}\right\}$, and $Q_{\alpha} \sigma=E_{i}$ for some $\alpha \in\left\{\gamma, \gamma^{\prime}\right\}$.

Proof. Let $W=E_{\mu}$ and $P=Q_{\gamma} \sigma$. Suppose that $P=W$. Then for each $w \in W$, there exists $u \in Q_{\gamma}$ such that $w=u \sigma$, or equivalently there exists $v \in E_{\mu^{\prime}}$ such that $u=w v$. Thus

$$
\omega\left(w \pi_{\gamma}\right)=\omega\left(u \pi_{\gamma} v^{-1} \pi_{\gamma}\right)=\omega\left(v^{-1} \pi_{\gamma}\right) \in \omega E_{\mu^{\prime}} \pi_{\gamma} .
$$

Therefore $\omega E \pi_{\gamma}=\omega E_{\mu^{\prime}} \pi_{\gamma}$, so by Lemma 5.3(2), $V=E_{\mu^{\prime}} \pi_{\gamma}$ is transitive on $\Gamma=\Gamma_{\gamma}$. Then as $W \pi_{\gamma}$ centralizes $V$, it follows that $W \pi_{\gamma}$ is semiregular on $\Gamma$, so (1) holds.

Now assume the hypothesis of (2). Suppose first that

$$
W_{\omega}<P<W .
$$


As $\gamma$ is $N_{H_{\omega}}(W)$-invariant, so are $Q_{\gamma}$ and $P$. However, if $H$ is semisimple then $\operatorname{Aut}_{H_{\omega}}(W)$ is maximal in $\operatorname{Aut}_{H}(W)$, contrary to (5.1). Similarly, if $H$ is complemented then $\operatorname{Inn}(W) \leq \operatorname{Aut}_{H_{\omega}}(W)$, again contrary to (5.1). Thus (5.1) fails, which establishes the first statement in (2). Furthermore, $W_{\omega} \leq Q_{\gamma} \cap Q_{\gamma^{\prime}}$, so $W_{\omega}=W_{\omega} \sigma \leq P \cap R$, where $R=Q_{\gamma^{\prime}} \sigma$. Thus if the second statement fails, then $P=R=W_{\omega}$ by the first statement, whereas $W=P R$ by Lemma 5.3(1). This contradiction completes the proof of (2).

LeMma 5.8. Assume that $H$ is semisimple, let $L=E_{1}, \beta=\beta_{1}$, take $1 \in \beta$, let $A$ be the stabilizer in $L$ of $\omega \in \Gamma_{1}$ under the representation $\pi_{1}: L \rightarrow X_{1} \leq \operatorname{Sym}\left(\Gamma_{1}\right)$, let $\sigma: E \rightarrow L$ be the projection map with respect to the direct sum decomposition $E=L \times E_{1^{\prime}}$, and set $c=\left|L: L_{\omega}\right|$ and $d=|L: A|$.

(1) $|\Omega|=c^{r}$.

(2) $N_{H}(L)$ is transitive on $\beta$.

(3) $b \leq 2$.

(4) Assume that $b=2$. Then $c=d^{2}, A=Q_{1} \sigma,\left|N_{H_{\omega}}(L): N_{H_{\omega}}(A)\right|=2$, and one of the following statements holds:

(i) $L \cong A_{6}, A \cong A_{5}, L_{\omega} \cong D_{10}$, and $d=6$;

(ii) $L \cong M_{12}, A \cong M_{11}, L_{\omega} \cong L_{2}(11)$, and $d=12$;

(iii) $L \cong S p_{4}(q)$ for some $q>2$ even, $A \cong O_{4}^{-}(q), L_{\omega}$ is an extension of $\mathbf{Z}_{q^{2}+1}$ by $\mathbf{Z}_{4}$, and $d=q^{2}\left(q^{2}-1\right) / 2$.

Proof. Part (1) follows from Lemma 2.2(3i). Assume that (2) fails and let $\gamma$ be an orbit of $K=N_{H}(L)$ on $\beta$. As $\varnothing \neq \gamma \subset \beta, \pi_{\alpha}$ is an injection on $L$ for $\alpha \in\left\{\gamma, \gamma^{\prime}\right\}$, so $1 \neq L_{\omega} \pi_{\alpha} \leq Q_{\alpha}$. In particular, $L \pi_{\alpha}$ is not semiregular on $\Gamma_{\alpha}$, so as $\alpha$ is $K$-invariant, Lemma 5.7 supplies a contradiction, completing the proof of (2).

Suppose that $b>1$. By Lemma 5.3(1), $L=P P^{\prime}$, where $P=Q_{1} \sigma$ and $P^{\prime}=Q_{1^{\prime}} \sigma$. By (2), $K$ is transitive on $\beta$, and as $E$ is transitive on $\Omega, K=E K_{\omega}$, so $J=K_{\omega}$ is transitive on $\beta$. Therefore $P^{J}=\left\{Q_{i} \sigma \mid i \in \beta\right\}$. Also for $j \in I-\beta, L \leq Q_{j}$, so $Q_{j} \sigma=L$. Thus as

$$
Q_{1^{\prime}}=\bigcap_{i \in 1^{\prime}} Q_{i}
$$

it follows that

$$
P^{\prime} \leq \bigcap_{1 \neq i \in \beta} Q_{i} \sigma
$$

As in the previous paragraph, $L \pi_{\alpha}$ is not semiregular on $\Gamma_{\alpha}$ for $\varnothing \neq \alpha \subset \beta$, so by Lemma 5.7(1), $P<L$. Then as $L=P P^{\prime}$, (5.2) says that $Q_{i} \sigma \neq P$ for $1 \neq i \in \beta$, so as $P^{J}=\left\{Q_{i} \sigma \mid i \in \beta\right\}$, it follows that

The map $i \mapsto Q_{i} \sigma$ is a bijection of $\beta$ with $P^{J}$. 
Furthermore, if $L_{\omega}=P$ then as $L_{\omega} \unlhd J, P^{J}=\{P\}$, contradicting (5.3) and $b>1$. Thus $L_{\omega}<P$. On the other hand, by Lemma 2.2(3i), $\operatorname{Aut}_{J}(L)=\operatorname{Aut}_{H_{\omega}}(L)$ is maximal in $\operatorname{Aut}_{H}(L)=\operatorname{Aut}_{K}(L)$. Thus $\left(\operatorname{Aut}_{K}(L), \operatorname{Aut}_{J}(L), L, P, b\right)$ satisfies the hypothesis in Lemma 3.4 on $(G, M, L, A, v)$, so (4) follows from that lemma, once we show that $P$ is the group $A$ defined in this lemma. But $Q_{1}$ is the stabilizer in $E$ of $\omega \in \Gamma_{1}$ under the representation $\pi_{1}: E \rightarrow \operatorname{Sym}\left(\Gamma_{1}\right)$, so $A=L \cap Q_{1}$ and $L_{\omega} \leq Q_{1}$. As $Q_{1}$ acts on $L$ as its projection $P,\left\langle L_{\omega}^{P}\right\rangle=\left\langle L_{\omega}^{Q_{1}}\right\rangle \leq L \cap Q_{1}=A \leq P$. Finally, from the list of groups in Lemma 3.4, $P=\left\langle L_{\omega}^{P}\right\rangle$, so $P=A$, completing the proof.

Lemma 5.9. Assume that $H$ is semisimple and $b=2$. Define $d$ as in Lemma 5.8.

(1) $m=d^{s}$ and $|\Omega|=d^{2 r}=d^{k s}$.

(2) $N_{H}\left(X_{1}\right)$ is transitive on $S_{1}$.

(3) $N_{H}\left(X_{1}\right)$ is primitive on $\Gamma_{1}$ with $\operatorname{Aut}_{H}\left(X_{1}\right)$ semisimple and $F^{*}\left(\operatorname{Aut}_{H}\left(X_{1}\right)\right)=$ $\operatorname{Aut}_{E}\left(X_{1}\right)$ is the direct product of the s copies Aut $_{E_{i}}\left(X_{1}\right), i \in S_{1}$, of $E_{1}$.

(4) Either

(i) $X_{1}$ is the alternating group $A_{m}$ on $\Gamma_{1}$; or

(ii) $s=1, m=d$, and $\mathcal{B}=\left\{\beta_{i} \mid i \in \bar{I}\right\}$ is a system of imprimitivity for $H$ on $I$. In addition, either:

(a) $X_{1} \cong E_{1}$; or

(b) $E_{1} \cong S p_{4}(q), d=q^{2}\left(q^{2}-1\right) / 2, q=q_{0}^{e}$ for some integer $e>1$, $X_{1} \cong \operatorname{Sp}_{4 e}\left(q_{0}\right)$, and $X_{1, \omega} \cong O_{4 e}^{-}\left(q_{0}\right)$.

ProOF. Adopt the notation from Lemma 5.8; in particular, $c=d^{2}$. For $i \in \bar{I}$, let $F_{i}=E_{i} \pi_{1}$ and set $F=\left\langle F_{i}: i \in S_{1}\right\rangle$. Then $E \pi_{1}=F$, so $F$ is transitive on $\Gamma=\Gamma_{1}$ by Lemma 5.3(2).

Next $N_{H}\left(E_{1}\right)$ is transitive on $\beta$ by Lemma 5.8(2), so $N_{H}\left(X_{1}\right)$ is transitive on $S_{1}$, establishing (2). From Lemma 5.8(4) and the proof of Lemma 3.4, $F_{i, \omega}$ is maximal in $F_{i}$. Thus as the projection of $F_{\omega}$ on $F_{i}$ acts on $F_{i, \omega}$, it follows that

(5) $F_{\omega}$ is the product of the groups $F_{i, \omega}, i \in S_{1}$, and $F_{i, \omega}$ is maximal in $F_{i}$.

By definition, $\left|F_{i}: F_{i, \omega}\right|=d$. Thus as $F$ is transitive on $\Gamma$, it follows from (5) that $m=\left|F: F_{\omega}\right|=d^{s}$, so (1) follows from Lemmas 5.8(1) and 5.4(2). Also (3) follows from (2) and (5), and the transitivity of $F$ on $\Gamma$. Then by Lemma 4.4, either (4i) holds or $s=1$, and we may assume the latter. Then $m=d$ by (1), and visibly $\mathcal{B}$ is a system of imprimitivity for $H$ on $I$. Without loss, $1 \in S_{1}$. Then from the main theorem of [LPS1], either $N_{\operatorname{Sym}(\Gamma)}\left(F_{1}\right)$ is the unique maximal overgroup of $F_{1}$ in $\operatorname{Sym}(\Gamma)$, so that $(4 \mathrm{i})$ or (4iia) holds, or (4iib) holds. This completes the proof of (4) and the lemma.

Definition 5.10. Define a semisimple group $H$ to be product decomposable if for $L$ a component of $H, c=\left|L: L_{\omega}\right|$, and $c=d^{2}$, one of the following statements holds: 
(i) $L \cong A_{6}, L_{\omega} \cong D_{10}$, and $d=6$;

(ii) $L \cong M_{12}, L_{\omega} \cong L_{2}(11)$, and $d=12$;

(iii) $L \cong S p_{4}(q)$ for some $q>2$ even, $L_{\omega}$ is an extension of $\mathbf{Z}_{q^{2}+1}$ by $\mathbf{Z}_{4}$ with $A \in \mathcal{O}_{L}\left(L_{\omega}\right)$ isomorphic to $O_{4}^{-}(q)$, and $d=q^{2}\left(q^{2}-1\right) / 2$.

Define a semisimple group $H$ to be product indecomposable if $H$ is not product decomposable.

Observe that $\mathcal{O}_{L}\left(L_{\omega}\right)$ contains two members $A_{i}, i=1,2$, of index $d$ in $L$, as described in Lemma 5.8(4). Furthermore, as $H$ is semisimple, $\operatorname{Aut}_{H_{\omega}}(L)$ is maximal in $\operatorname{Aut}_{H}(L)$ by Lemma 2.2, so $N_{H_{\omega}}(L)$ is transitive on $\left\{A_{1}, A_{2}\right\}$, and hence $\mid N_{H_{\omega}}(L)$ : $N_{H_{\omega}}\left(A_{1}\right) \mid=2$.

Observe from Lemma 5.8 that if $H$ is almost simple then $H$ is product decomposable precisely when $H$ preserves a nontrivial product structure on $\Omega$. We will see in the next lemma that the general semisimple group $H$ is product decomposable if $\mathcal{F}(H)$ can be composed with the rank-two product structure preserved by a component of $H$ (using the composition defined in Definition 1.11) to produce a larger $H$-invariant product structure $\mathcal{F}^{2}(H)$.

LEMMA 5.11. Assume that $H$ is semisimple and product decomposable. Let $\hat{I}=$ $\{1,2\}$ and $\tilde{I}=\hat{I} \times \bar{I}$. Set $\Sigma_{i}=\omega E_{i}$.

(1) $H$ preserves the regular $(c, r)$-product structure $\overline{\mathcal{F}}=\mathcal{F}(H)$ on $\Omega$.

(2) For $i \in \bar{I}, E_{i} N_{H_{\omega}}\left(E_{i}\right)$ preserves a unique product structure $\hat{\mathcal{F}}_{i}$ on $\Sigma_{i}$. Indeed, $\hat{\mathcal{F}}_{i}=\left(\Sigma_{i, 1}, \Sigma_{i, 2}\right)$ is the $(d, 2)$-structure defined by $\Sigma_{i, j}=\Sigma_{i, j}(\omega) A_{i, 3-j}$ and $\Sigma_{i, j}(\omega)=\omega A_{i, j}$, where $\left\{A_{i, 1}, A_{i, 2}\right\}$ are the maximal overgroups of $E_{i, \omega}$ of index $d$ in $E_{i}$ described in Lemma 5.8(4).

(3) $H$ preserves the $(d, 2 k)$-product structure $\tilde{\mathcal{F}}=\left(\Omega_{i, j}:(i, j) \in \tilde{I}\right)$ on $\Omega$, defined by $\Omega_{i, j}=\left\{\Sigma_{i, j}(\omega) D_{i^{\prime}} g \mid g \in A_{i, 3-j}\right\}$. Furthermore, $\tilde{\mathcal{F}}=\hat{\mathcal{F}}_{1} \circ \overline{\mathcal{F}}$.

(4) Let $\tilde{M}=N_{G}(\tilde{\mathcal{F}}), \tilde{X}=F^{*}(\tilde{M})$, and for $(i, j) \in \tilde{I}$ let $X_{i, j}$ be the component of $\tilde{M}$ fixing all blocks in $\Omega_{u, v}$ for all $(u, v) \in \tilde{I}-\{(i, j)\}, Y_{i}=N_{H_{\omega}}\left(A_{i, 1}\right) \cap$ $N_{H_{\omega}}\left(A_{i, 2}\right)$, and $K_{i}=Y_{i} E$. Then $E_{i} \leq X_{i, 1} \times X_{i, 2}$ and $K_{i}=N_{H}\left(X_{i, j}\right)$.

(5) Suppose that $b=2$, for $i \in \bar{I}$ let $\beta_{i}=\left\{i_{1}, i_{2}\right\}$, and for $(i, j) \in \tilde{I}$ let $P_{i, j}$ be the projection of $E$ on $X_{i, j}$ and $E_{i, j}=E_{i} \pi_{i_{j}}$. Then we can choose notation so that $P_{i, j}=E_{i, j}$.

(6) Identify the $H$-sets $\tilde{I}$ and $\left\{X_{i, j} \mid(i, j) \in \tilde{I}\right\}$ as in Notation 5.2. For $\gamma \subseteq \tilde{I}$, set $E_{\gamma}=\left\langle P_{i, j}:(i, j) \in \gamma\right\rangle$ and $\gamma^{\prime}=\tilde{I}-\gamma$. For $K \in \mathcal{O}_{H}\left(K_{1}\right)$, let $\gamma_{K}=(1,1) K$. Then $\mathcal{P}_{K}=\gamma_{K}^{H}$ is a partition of $\tilde{I}$. Set $\mathcal{E}_{K}=\left\{E_{\gamma} \mid \gamma \in \mathcal{P}_{K}\right\}$ and $\mathcal{F}^{2}(H, K)=$ $\mathcal{F}\left(\mathcal{E}_{K}\right)$. Then $\mathcal{F}^{2}(H, K)$ is an $\left(m_{K}, r_{K}\right)$-product structure on $\Omega$, where $r_{K}=$ $|H: K|$ and $m_{K}=d^{\left|K: K_{1}\right|}$, and the map $\phi: K \mapsto \mathcal{F}^{2}(H, K)$ is a bijection of $\mathcal{O}_{H}\left(K_{1}\right)^{\prime}=\mathcal{O}_{H}\left(K_{1}\right)-\{H\}$ with $\mathcal{F}(H)$. Further, $\mathcal{F}^{2}(H)=\mathcal{F}^{2}\left(H, K_{1}\right)=\tilde{\mathcal{F}}$ and $b\left(N_{G}\left(\mathcal{F}^{2}(H, K)\right), H\right)=1$ if and only if $N_{H}\left(E_{1}\right) \leq K$. 
PROOF. Part (1) is a consequence of Notation 2.6.

From Definition 5.10, $N_{H_{\omega}}\left(E_{i}\right)$ acts transitively on $\left\{A_{i, 1}, A_{i, 2}\right\}$ via conjugation. Thus $N_{H_{\omega}}\left(E_{i}\right)=Y_{i}\left\langle t_{i}\right\rangle$, where $t_{i}$ fixes $\omega$ and interchanges $A_{i, 1}$ and $A_{i, 2}$. Thus $\omega A_{i, j} t_{i}=\omega A_{i, j}^{t_{i}}=\omega A_{i, 3-j}$, so $t_{i}$ interchanges $\Sigma_{i, 1}$ and $\Sigma_{i, 2}$. As $E_{i}=A_{i, 1} A_{i, 2}, E_{i}$ permutes the blocks in $\Sigma_{i, j}$ for $j \in \hat{I}$. As $Y_{i}$ fixes $\omega$ and acts on $A_{i, j}, Y_{i}$ fixes the blocks. Thus $J_{i}=E_{i} Y_{i}$ permutes the blocks, so as $t_{i}$ interchanges $\Sigma_{1,1}$ and $\Sigma_{i, 2}$, $\hat{J}_{i}=E_{i} N_{H_{\omega}}\left(E_{i}\right)=J_{i}\left\langle t_{i}\right\rangle$ preserves $\hat{\mathcal{F}}_{i}$. Moreover, this argument shows that $J_{i}$ is the subgroup of index 2 in $\hat{J}_{i}$ which permutes $\Sigma_{i, j}$ for $j \in \hat{I}$. As the factorization $E_{i}=A_{i, 1} A_{i, 2}$ is determined up to conjugation in $E_{i}$ and the ordering of the factors, the product structure $\hat{\mathcal{F}}_{i}$ is unique, completing the proof of (2). Notice also that $N_{H}\left(E_{i}\right)=E N_{H_{\omega}}\left(E_{i}\right)=E \hat{J}_{i}$, so $\left|N_{H}\left(E_{i}\right): K_{i}\right|=\left|E \hat{J}_{i}: E Y_{i}\right|=\left|\hat{J}_{i}: J_{i}\right|=2$.

From the previous paragraph, the hypothesis of Lemma 1.13 is satisfied, so by that lemma $\tilde{\mathcal{F}}$ is the composition of the structures $\overline{\mathcal{F}}$ and $\hat{\mathcal{F}}_{1}$, and (3) holds.

From the description of $\tilde{\mathcal{F}}$ in (3), $E_{i}$ acts on each block of $\Omega_{u, v}$ for $(u, v) \in$ $\tilde{I}-\{(i, j)\}$, so $E_{i} \leq X_{i, 1} \times X_{i, 2}$. Thus as $H$ permutes $\mathcal{E}$ and the components $X_{i, j}$, $N_{H}\left(X_{i, j}\right) \leq N_{H}\left(E_{i}\right)$. Then $K_{i}=E J_{i}=N_{H}\left(X_{i, j}\right)$, as $J_{i}$ is the stabilizer in $N_{H_{\omega}}\left(E_{i}\right)$ of $\Sigma_{i, j}$, establishing (4).

Assume the hypothesis of (5) and let $\pi_{i, j}: \tilde{X} \rightarrow X_{i, j}$ be the projection map. As $E_{i} \leq X_{i, 1} \times X_{i, 2}, P_{i, j}=E \pi_{i, j}=E_{i} \pi_{i, j} \cong E_{i}$. Then $P_{i, j}$ is characterized by the property that it is trivial on all blocks of $\tilde{\mathcal{F}}$ except for those in $\Omega_{i, j}$, and is isomorphic to $E_{i}$. As $E_{i, j}$ centralizes $E_{i^{\prime}}$ and $E_{i, j, \omega} \neq 1$ acts on $\Sigma_{u, v}(\omega)$ for each $(u, v) \in \tilde{I}$ with $u \neq i, E_{i, j}=\left[E_{i, j}, E_{i, j, \omega}\right]$ fixes all fibers not in $\Sigma_{i, l}$ for some $l \in \hat{I}$. Furthermore, from Example 1.6, the orbits of $E_{i, 1}$ and $E_{i, 2}$ on $\Sigma_{i}$ form a product structure on $\Sigma_{i}$, so from (2) this structure is $\hat{\mathcal{F}}$. Thus we may choose notation such that $E_{i, j} \cong E_{i}$ is trivial on all blocks of $\tilde{\mathcal{F}}$ except those in $\Sigma_{i, j}$, so that $E_{i, j}=P_{i, j}$, completing the proof of (5).

Let $K \in \mathcal{O}_{H}\left(K_{1}\right)$. By (4), $K_{1}=N_{H}\left(X_{1, j}\right)$, so $K_{1}$ is the stabilizer of $(1, j) \in \tilde{I}$ in $H$. Thus as $K_{1} \leq K, \mathcal{P}_{K}$ is a partition of $\tilde{I}$. Hence by Example $1.6, \mathcal{F}^{2}(H, K)$ is an $H$-invariant $\left(m_{K}, r_{K}\right)$-product structure on $\Omega$. Visibly the map $\phi$ is injective. Thus it remains to show that $\phi$ is surjective. Hence we may assume that $M$ is the stabilizer in $G$ of $\mathcal{F}$, and it suffices to show that $\mathcal{F}=\mathcal{F}^{2}(H, K)$, where $K=N_{H}\left(X_{1}\right)$. Pick notation so that $E_{1}$ projects nontrivially on $X_{1}$.

First, $b=1$ if and only if $E_{1} \leq X_{1}$ if and only if $N_{H}\left(E_{1}\right) \leq K$ by Lemma 5.8(2). In that event, since $K_{1} \leq N_{H}\left(E_{1}\right)$, then $K_{1} \leq K$, so indeed $K \in \mathcal{O}_{H}\left(K_{1}\right)$. Furthermore, we saw earlier that $\left|N_{H}\left(E_{1}\right): K_{1}\right|=2$, so $\gamma_{K}=\left\{(i, j) \mid E_{i} \leq X_{1}\right\}$, and hence $E_{\gamma_{K}}=\left\langle E_{i}: E_{i} \leq X_{1}\right\rangle$. Thus $\omega X_{1}=\omega E_{\gamma_{K}}$, so indeed $\mathcal{F}^{2}(H, K)=\mathcal{F}$ by Lemma 1.7. Thus we may assume that $b=2$.

By (5), $E_{1,1}=E_{1} \cap X_{1,1}$ and $K_{1}=N_{H}\left(X_{1,1}\right)$, so $E_{1,1}$ is $K_{1}$-invariant. Thus $K_{1} \leq K$, so again $K \in \mathcal{O}_{H}\left(K_{1}\right)$. By Lemma 5.9(2), $K$ is transitive on $S_{1}$, so $\gamma_{K}=\left\{(i, j(i)) \mid i \in S_{1}\right\}$ for some function $j: S_{1} \rightarrow \hat{I}$ such that $\Gamma_{1}=\omega F$, where $F=\left\langle E_{i, j(i)}: i \in S_{1}\right\rangle$. Then $\Gamma_{1}=\omega F=\omega E_{\gamma_{K}}$ as $E_{i, j(i)}=P_{i, j}$ by (5). Thus $\mathcal{F}=\mathcal{F}^{2}(H, K)$, completing the proof. 
Lemma 5.12. Assume that $H$ is semisimple and product indecomposable. Then:

(1) $b=b(M, H)=1$ for each $M \in \mathcal{O}_{G}(H)$ which is semisimple;

(2) the map $K \mapsto \mathcal{F}(H, K)$ is a bijection of $\mathcal{O}_{H}\left(N_{H}\left(E_{1}\right)\right)^{\prime}$ with $\mathcal{F}(H)$.

Proof. As $H$ is indecomposable, (1) follows from parts (3) and (4) of Lemma 5.8. As defined in Notation 2.6, $\mathcal{F}(H, K)$ is an $H$-invariant product structure. If $\mathcal{F}^{\prime}$ is an $H$ invariant product structure, then $H \leq M^{\prime}=N_{G}\left(\mathcal{F}^{\prime}\right)$, and $M^{\prime}$ is semisimple. Then by (1) and Lemma 5.5(6), $\mathcal{F}^{\prime}=\mathcal{F}(H, K)$, where $K=N_{H}\left(X_{1}^{\prime}\right)$ and $X_{1}^{\prime}$ is the component of $F^{*}\left(M^{\prime}\right)$ containing $E_{1}$. By Lemma 5.3(4), $K \neq H$, so the map $\phi$ of (2) is surjective. Visibly $\phi$ is injective, completing the proof.

Lemma 5.13. Assume that $H$ is complemented and maximal in $M$. Then $b=1$.

Proof. Let $\rho: M \rightarrow \operatorname{Sym}(M / H)$ be the representation of $M$ on $M / H$ via right multiplication. Suppose that $K=\operatorname{ker}(\rho) \neq 1$. Then as $X$ is the unique minimal normal subgroup of $M, X \leq K \leq H \leq N_{M}(E)$. By Lemma 4.3, $E \leq X$, so $E \unlhd X$. Then as $C_{H}(E)=1, E=X$, contradicting $E_{\omega}=1 \neq X_{\omega}$.

Therefore $\rho$ is faithful. As $H$ is maximal in $M, M$ is primitive on $M / H$. Thus, applying Lemma 2.2 to $\rho$ and recalling that $X=E(M)$ is the unique minimal normal subgroup of $M$, we conclude that $M$ is semisimple, complemented, or diagonal on $M / H$. As $1 \neq E \leq H \cap M, M$ is not complemented. Suppose that $M$ is semisimple on $M / H$. Then $H \cap X$ is the direct product of the groups $H \cap X_{i}, i \in I$. Let $j \in S_{i}$. Then $1 \neq\left[H \cap X_{i}, E_{j}\right]$, so $E_{j} \leq\left[H \cap X_{i}, E_{j}\right] \leq X_{i}$, so $b=1$ and the lemma holds. Thus we may assume that $M$ is diagonal on $M / H$. Hence there is an $H$-invariant partition $\Delta$ of $I$ with $H \cap X$ the product of full diagonal subgroups $F_{\delta}$ of $X_{\delta}, \delta \in \Delta$. Therefore $\mathcal{E}=\left\{F_{\delta} \mid \delta \in \Delta\right\}, E_{1} \cong X_{1}, s=1$, and $b=|\delta|$ for $\delta \in \Delta$. By Lemma 3.5 we may pick $p \in \pi\left(X_{1}\right)$ with $m_{p}\left(X_{1}\right)=1$. Then by Lemma 3.6,

$$
k=m_{p}(X)=m_{p}(E)+m_{p}\left(X_{\omega}\right)=r+k m_{p}\left(X_{1, \omega}\right),
$$

contradicting $k=r b$ and $b>1$.

\section{Diagonal overgroups of primitive groups}

In this section we make the following assumption.

Hypothesis 6.1. Hypothesis 2.1 is satisfied and $H$ is not affine. Furthermore, $M \in \mathcal{O}_{G}(H)$ and $M$ is diagonal.

Notation 6.2. Let $X=F^{*}(M), I=\{1, \ldots, k\}$, and $\bar{I}=\{1, \ldots, r\}$.

Let $\mathcal{X}=\left\{X_{i} \mid i \in I\right\}$ be the set of components of $M$. For $\gamma \subseteq I$, set $\gamma^{\prime}=I-\gamma$, $X_{\gamma}=\left\langle X_{i}: i \in \gamma\right\rangle, \Gamma_{\gamma}=\omega X_{\gamma}$, and $\pi_{\gamma}: X \rightarrow X_{\gamma}$ the projection map with respect to the direct sum decomposition $X=X_{\gamma} \times X_{\gamma^{\prime}}$. Set $c=\left|X_{i}\right|$.

Let $E$ be a minimal normal subgroup of $H$ and $\mathcal{E}=\left\{E_{i} \mid i \in \bar{I}\right\}$ the set of components of $E$. Represent $M$ on $I$ and $H$ on $\bar{I}$ so that the maps $i \mapsto X_{i}$ and 
$j \mapsto E_{j}$ are equivalences of permutation representations. As $H$ is transitive on $\mathcal{E}$, $H$ is transitive on $\bar{I}$.

As $M$ is diagonal, there exists a maximal $M$-invariant partition $\Sigma=\Sigma(M)$ of $I$ such that $X_{\omega}$ is the direct product of full diagonal subgroups $F_{\sigma}, \sigma \in \Sigma$, of $X_{\sigma}$. Recall that $M$ is strongly diagonal if $\Sigma=\{I\}$. In that event write $\mathbf{d}(M)$ for the diagonal structure $\operatorname{diag}\left(\mathcal{X}, X_{\omega}\right)$ defined by $M$ as in Definition 1.9. Let $k_{M}=|\Sigma|$ and $m_{M}=c^{|\sigma|-1}$ for $\sigma \in \Sigma$.

LEMMA 6.3. Assume that $M$ is not strongly diagonal and let $\mathcal{D}=\left\{X_{\sigma} \mid \sigma \in \Sigma\right\}$.

(1) $\mathcal{F}(M)=\mathcal{F}(\mathcal{D})$ is an $M$-invariant regular $\left(m_{M}, k_{M}\right)$-product structure on $\Omega$.

(2) Let $M^{\prime}=N_{G}\left(\mathcal{F}(M)\right.$ ) and let $Y_{\sigma}$ be the component of $M^{\prime}$ acting faithfully on the $\sigma$-factor $\Gamma_{\sigma}$ of the product structure. Then $\operatorname{Aut}_{M}\left(Y_{\sigma}\right)$ is strongly diagonal on $\Gamma_{\sigma}$.

PROOF. Part (1) follows from Example 1.6. By construction $\operatorname{Aut}_{X}\left(Y_{\sigma}\right)=X_{\sigma}$ is the direct product of $|\sigma|>1$ copies of $X_{1}$ with $X_{\sigma, \omega}=F_{\sigma}$ a full diagonal subgroup, and $N_{H}(\sigma)$ is primitive on $\sigma$, so (2) holds.

\section{LEMMA 6.4. Assume that $M$ is strongly diagonal.}

(1) $X_{\omega} \cong X_{1}$ is an $M_{\omega}$-invariant full diagonal subgroup of $X$.

(2) $|\Omega|=c^{k-1}$.

PROOF. This follows from Lemma 2.2.

Lemma 6.5. Assume that $H$ is doubled. Then $k=2 r, r=k_{M},|\sigma|=2$ for $\sigma \in \Sigma$, $X=D$, and:

(1) if $M$ is strongly diagonal then $r=1$ and $\mathbf{d}(M)=\mathbf{d}(H)$ as defined in Lemma 4.7(2);

(2) if $M$ is not strongly diagonal then $\mathcal{F}(M)=\mathcal{F}(H)$ as defined in Notation 2.6.

Proof. First assume that $M$ is strongly diagonal. As $H$ is doubled, $\left|D_{\omega}\right|=|E|=$ $|\Omega|$. Thus $\left|D_{\omega}\right|=c^{k-1}$ by Lemma $6.4(2)$. But by Lemma $4.3, D_{\omega} \leq X_{\omega}$ and by Lemma 6.4(1), $X_{\omega} \cong X_{1}$ is simple of order $c$. Therefore $k=2$ and $D_{\omega}=X_{\omega}$ is simple. Therefore $E$ is simple, so $r=1$ and $X=D$. Now Lemma 4.7(2) completes the proof in this case.

So assume that $M$ is not strongly diagonal, form the product structure $\mathcal{F}=\mathcal{F}(M)$ of Lemma 6.3, and adopt the notation of Lemma 6.3(2). By Lemma 6.3, $H \leq M \leq M^{\prime}$, so by Lemma $5.6(1), b^{\prime}=b\left(M^{\prime}, H\right)=1$. Then by Lemma 5.5(7), $\operatorname{Aut}_{H}\left(Y_{\sigma}\right)$ is doubled on $\Gamma_{\sigma}$ with $D \cap Y_{\sigma}$ the direct product $\left(E \cap Y_{\sigma}\right) \times\left(\tilde{E} \cap Y_{\sigma}\right)$, where $\tilde{E}$ is the second minimal normal subgroup of $H$. Now by Lemma 5.6(2), $\mathcal{F}=\mathcal{F}\left(H, H_{\sigma}\right)$, where $H_{\sigma}$ is the stabilizer in $H$ of $\sigma$. Moreover, $D \cap Y_{\sigma} \leq X \cap Y_{\sigma}=X_{\sigma}$, Aut $_{M}\left(Y_{\sigma}\right)$ is strongly diagonal on $\Gamma_{\sigma}$ by Lemma 6.3(2), and we saw that $\operatorname{Aut}_{H}\left(Y_{\sigma}\right)$ is doubled on $\Gamma_{\sigma}$, so by (1), $|\sigma|=2$ and $X_{\sigma}=D \cap X_{\sigma}$. Thus $k=|\sigma| k_{M}=2 r$ and $X=D$, and Lemma 4.7(1) completes the proof. 
LEMMA 6.6. If $H$ is diagonal then $D=X$ and $\Sigma(M)=\Sigma(H)$.

Proof. Assume that $H$ is diagonal, let $\Delta=\Sigma(H)$ be of order $k_{H}$, and set $e=\left|E_{1}\right|$. Then by Lemma 2.2(3ii),

$$
e^{r-k_{H}}=|\Omega|=c^{k-k_{M}},
$$

so $e$ and $c$ have the same set $\Pi$ of prime divisors. By Lemma 3.5, there exists $p \in \Pi$ such that $X_{1}$ has cyclic Sylow $p$-subgroups. Thus $m_{p}(X)=k$.

Suppose that $M$ is strongly diagonal. Then $X_{\omega} \cong X_{1}$, so $m_{p}\left(X_{1}\right)=1$. But $E_{\omega}$ is the direct product of $k_{H}$ copies of $E_{1}$, and $E_{\omega} \leq X_{\omega}$, so

$$
1=m_{p}\left(X_{\omega}\right) \geq m_{p}\left(E_{\omega}\right)=k_{H} m_{p}\left(E_{1}\right) .
$$

It follows that $k_{H}=1$ and $m_{p}\left(E_{1}\right)=1$. Thus $H$ is strongly diagonal.

Next the product $J$ of $r-1$ components of $E$ is a regular normal subgroup of $E$, so by Lemma 3.6(2),

$$
k=m_{p}(X)=m_{p}(J)+m_{p}\left(X_{\omega}\right)=(r-1) m_{p}\left(E_{1}\right)+1=r .
$$

Thus $k=r$, so it follows from (6.1) that $c=e$. Then $|X|=c^{k}=e^{r}=|E|$, so $X=E=D$ and as both $H$ and $M$ are strongly diagonal, also $\Sigma(H)=\Sigma$. Thus the lemma holds in this case.

So assume $k_{M}>1$, form the product structure $\mathcal{F}=\mathcal{F}(M)$ of Lemma 6.3, and adopt the notation of Lemma 6.3(2). Let $I_{H}=\bar{I}, I_{M}=I$, and for $U \in\{H, M\}$ and $\sigma \in \Sigma$, define $S_{U, \sigma}=\left\{j \in I_{U} \mid F^{*}(U)_{j} \leq X_{\sigma}\right\}$ and the partition $\mathcal{S}_{U}=\left\{S_{U, \sigma} \mid \sigma \in \Sigma\right\}$ of $I_{U}$ as in Notation 5.2 and Lemma 5.5(3). By construction in Lemma 6.3, $S_{M, \sigma}=\{\sigma\}$, so $\mathcal{S}_{M}=\Sigma$. From Lemma 5.6(1), $b^{\prime}=b\left(M^{\prime}, H\right)=1$, and then from Lemma 5.5(8), $\operatorname{Aut}_{H}\left(Y_{\sigma}\right)$ is diagonal on $\Gamma_{\sigma}$, with $\Delta=\Sigma(H)$ a refinement of $\mathcal{S}_{H}$. As usual, $\operatorname{Aut}_{H}\left(Y_{\sigma}\right) \leq \operatorname{Aut}_{M}\left(Y_{\sigma}\right)$, which is strongly diagonal on $\Gamma_{\sigma}$ by Lemma 6.3(2). Thus from the case treated above, $X_{\sigma}=D \cap Y_{\sigma}$ and $\operatorname{Aut}_{H}\left(Y_{\sigma}\right)$ is strongly diagonal. Therefore $X=D$ and $N_{H}\left(Y_{\sigma}\right)$ is primitive on $\sigma$, so $\Sigma=\Delta$ as $\Delta$ is a refinement of $\mathcal{S}_{H}$. Thus the lemma holds in this case too.

In the next lemma, for $K \in\{H, M\}, \mathcal{P}(K)$ is the set of partitions of the components of a minimal normal subgroup of $K$ defined in Notation 2.6.

Lemma 6.7. Assume that $H$ is complemented. Then $k=2 r, r=k_{M}, E \unlhd X=$ $E C_{G}(E)$, and $\mathcal{P}(H)=\mathcal{P}(M)$.

ProOf. Assume otherwise. For $i \in \bar{I}$ let

$$
\beta_{i}=\left\{j \in I \mid E_{i} \pi_{j} \neq 1\right\}
$$

and for $i \in I$ let $S_{i}=\left\{j \in \bar{I} \mid E_{j} \pi_{i} \neq 1\right\}$. As $H$ is transitive on $\bar{I}, b=\left|\beta_{i}\right|$ is independent of $i \in \bar{I}$. For $\alpha \subseteq \bar{I}$, let $E_{\alpha}=\left\langle E_{i}: i \in \alpha\right\rangle$. 
Let $e=\left|E_{i}\right|$. Then

(1) $e^{r}=|\Omega|=c^{k-k_{M}}$,

so the set $\Pi$ of primes dividing $e$ and $c$ are the same. By Lemma 3.5, there exists $p \in \Pi$ with $m_{p}\left(X_{1}\right)=1$. Thus as $\pi_{i}: E_{S_{i}} \rightarrow X_{i}$ is an injection for each $i \in I$, it follows that (2) for all $i \in I,\left|S_{i}\right| \leq 1$,

and

(3) for all $j \in \bar{I}, m_{p}\left(E_{j}\right)=1$.

It follows from (2) that

(4) $\mathcal{B}=\left\{\beta_{j} \mid j \in \bar{I}\right\}$ is a partition of $I^{\prime}=\left\{i \in I|| S_{i} \mid=1\right\}$, so $\left|I^{\prime}\right|=r b$; and

(5) $r=k-k_{M}$.

Namely by (3), $m_{p}(E)=r$, while by Lemma 3.6(2),

$$
k=m_{p}(X)=m_{p}(E)+m_{p}\left(X_{\omega}\right)=r+k_{M},
$$

establishing (5). Then by (1) and (5), $c=e$, so as $\pi_{i}: E_{1} \rightarrow X_{i}$ is injective for $i \in \beta_{1}$, (6) $\quad E_{1} \cong X_{1}$ and $c=e$.

Let $u=|\sigma|$ for $\sigma \in \Sigma$; thus $k=k_{M} u$. We next show that

(7) either $b=1$, or $b=u=2, k=2 r, r=k_{M}$, and $I=I^{\prime}$.

Namely by (4) and (5), $r b \leq k=r+k_{M}$, so $k_{M} \geq r(b-1)$. Furthermore, $k=k_{M} u$, so $r=k_{M}(u-1)$ by (5). Thus $k_{M} \geq k_{M}(u-1)(b-1)$, so as $u>1$, it follows that either $b=1$ or $b=u=2$ and all inequalities are equalities. That is, (7) holds.

(8) Either

(i) $H$ is transitive on $I$, or

(ii) $u=b=2, k=2 r, r=k_{M}, I=I^{\prime}, H$ has orbits $\gamma$ and $\gamma^{\prime}$ on $I$ of length $r$, and $E$ is a full diagonal subgroup of $X_{\gamma} \times X_{\gamma^{\prime}}$.

For assume that $\gamma \neq I$ is an orbit of $H$ on $I$. Then $X_{\gamma}$ and $X_{\gamma^{\prime}}$ are normal in $H X$, so $H X$ has at least two minimal normal subgroups. But $H X$ is primitive by Lemma 2.4, so from Lemma 2.2, $H X$ is doubled and $X_{\alpha}$ is regular on $\Omega$ for $\alpha \in\left\{\gamma, \gamma^{\prime}\right\}$. It follows from (1) that $k_{M}=|\gamma|$ and $k=2 k_{M}$, so $u=2$. Next as $E$ is a minimal normal subgroup of $H$, either $E \cap X_{\gamma}=1$ or $E \leq X_{\gamma}$. But as $|E|=\left|X_{\gamma}\right|$ by (1), in the latter case $E=X_{\gamma}$. Then $\mathcal{P}(M)=\mathcal{P}(H)$, the product structure in Notation 2.6, and $X=E C_{G}(X)$ by Lemma 4.6, contrary to the choice of $M$ as a counterexample. Hence $b \neq 1$, so (ii) holds by (7).

(9) $u=b=2, k=2 r, r=k_{M}$, and $I=I^{\prime}$. 
If not, then $b=1$ and $H$ is transitive on $I$ by (7) and (8). As $b=1$, we may take $E_{1} \leq X_{1}$, so $E_{1}=X_{1}$ by (6). Then as $H$ is transitive on $I, E=X$ and $r=k$, contrary to (1).

(10) $M=H X$ and $H$ is transitive on $I$.

For $H \leq H X=\tilde{M}$, and by Lemma 2.4, $\tilde{M}$ is primitive on $\Omega$. As $M$ is diagonal, $X_{\omega}$ is the product of full diagonal subgroups of the groups $X_{\sigma}, \sigma \in \Sigma$. If $H$ is transitive on $I$ then $X$ is the unique minimal normal subgroup of $\tilde{M}$, so $\tilde{M}$ is diagonal, and hence as $b \neq 1, M=\tilde{M}$ by induction on $|M|$. On the other hand, if $M$ is not transitive on $I$ then $\tilde{M}$ has at least two minimal normal subgroups, so $\tilde{M}$ is doubled. Then applying Lemma 8.1 (whose proof does not depend upon this lemma) to $\tilde{M}$, we conclude that $D \unlhd X$, again contradicting $b \neq 1$.

For $i \in I$, let $\sigma_{i}$ be the block in $\Sigma$ containing $i$. Define a graph $\mathcal{G}$ on $\Sigma$ by $\sigma$ adjacent to $\mu$ if $\sigma \cap \beta_{i} \neq \varnothing \neq \mu \cap \beta_{i}$ for some $i \in \bar{I}$. Let $\Delta$ be a connected component of $\mathcal{G}$, $J=\left\{i \in I \mid \sigma_{i} \in \Delta\right\}$ and $\bar{J}=\left\{i \in \bar{I} \mid \beta_{i} \subseteq J\right\}$. For $i \in \bar{I}$, let $K_{i}=O^{2}\left(H_{i}\right)$.

(11) $|J|=2|\bar{J}|, K_{i}=K$ is independent of $i \in \bar{J}$, and $K$ fixes $J$ and $\bar{J}$ pointwise.

Observe that $\Delta$ is a partition of $J$. Hence if $i \in \bar{I}$ with $\beta_{i} \cap J \neq \varnothing$, then $\beta_{i} \cap \sigma \neq \varnothing$ for some $\sigma \in \Delta$. Then as $\Delta$ is a connected component of $\mathcal{G}$, for each $\mu \in \Sigma$ with $\beta_{i} \cap \mu \neq \varnothing, \mu \in \Delta$. Thus $\beta_{i} \subseteq J$, so $i \in \bar{J}$. Hence by (4) and (9), $\left\{\beta_{i} \mid i \in \bar{J}\right\}$ is a partition of $J$. Next $I=I^{\prime}$ by (9), so the map $i \mapsto \beta_{i}$ is a bijection of $\bar{I}$ with $\mathcal{B}$. Thus as $b=2,|J|=2|\bar{J}|$. Let $i \in \bar{J}$ and $j \in \beta_{i}$. As $b=2, K_{i}$ fixes $\beta_{i}$ pointwise, and then as $u=2, K_{i}$ fixes $\sigma_{j}$ pointwise for $j \in \beta_{i}$. Then as $\Delta$ is connected, $K_{i}$ fixes $J$ and $\bar{J}$ pointwise. Hence for $j \in \bar{J}, K_{i} \leq O^{2}\left(H_{j}\right)=K_{j}$, so as $H$ is transitive on $\bar{I}, K_{i}=K_{j}$. This completes the proof of (11).

(12) $J \neq I$.

Suppose that $\bar{J}=\bar{I}$. Then by (11), $K$ fixes $\bar{I}$ pointwise, so $K^{\infty} \leq H_{\bar{I}}^{\infty}=D$. Now $Y=N_{H_{\omega}}\left(E_{1}\right)^{\infty} \leq H_{1}^{\infty}=K^{\infty} \leq D$. Thus $Y=1$ as $D$ is regular on $\Omega$, which is a contradiction as $\operatorname{Aut}_{Y}\left(E_{1}\right)=\operatorname{Inn}\left(E_{1}\right)$ by Lemma 2.2. Therefore $\bar{J} \neq \bar{I}$, so as $|I|=2|\bar{I}|$ by (9) and $|J|=2|\bar{J}|$ by (11), also $J \neq I$.

We now obtain a contradiction, establishing the lemma. Namely $H$ permutes the connected components of $\mathcal{G}$, so as $M=H X$ by (10), $M$ is transitive on those components, and hence $\Gamma=J^{M}$ is a partition of $I$. Then as $J \neq I$ by (12), $\Gamma \in \mathcal{P}(M)$. Therefore, from Notation 2.6 and Lemma 5.5(8), $N_{M}(J)$ acts primitively as a diagonal group $M^{\prime}$ on $\Omega^{\prime}=\omega M^{\prime}$. Similarly, by Lemma 5.5(9), $N_{H}(J)$ acts primitively as a complemented or doubled group $H^{\prime}$ on $\Omega^{\prime}$. In the first case, proceeding by induction on $|\Omega|, b=1$, contrary to (9). Therefore $N_{H}(J)$ is doubled on $\Omega^{\prime}$. Then from Lemma $6.5, X=E C_{X}(E)$, contradicting $b \neq 1$.

\section{The proofs of Propositions 5, 6, and 7}

In this section we assume Hypothesis 2.1. Refer to Example 1.6 and Notation 2.6 for the definitions of $\mathcal{F}(\mathcal{L}), \mathcal{F}(H)$, and $\mathcal{F}(H, K)$. 
LEMMA 7.1. Assume that $H$ is octal semisimple with components $\mathcal{L}=\left\{L_{1}, \ldots, L_{k}\right\}$.

(1) If $k>1$ then $N_{S}(D) \leq N_{S}(\mathcal{F}(\mathcal{L}))$ and $\mathcal{F}(\mathcal{L})=\mathcal{F}(H)$.

(2) $\quad N_{S}(D)=D U T$, where $U=\left\langle u_{1}, \ldots, u_{k}\right\rangle \cong E_{2^{k}}, u_{i}$ induces an outer automorphism on $L_{i}$ and centralizes $L_{j}$ for $j \neq i, T$ acts faithfully as $\operatorname{Sym}(\mathcal{L})$ on $\mathcal{L}$, and on $U$ as the $\mathbf{F}_{2} T$-permutation module, and $N_{S}(D)_{\omega}=D_{\omega} U T$.

(3) Let $\mathcal{H}$ be the set of primitive semisimple subgroups $\tilde{H}$ of $S$ such that $D=F^{*}(\tilde{H})$. Then $U$ acts regularly on the set $\mathcal{A}$ of affine structures invariant under some member of $\mathcal{H}$.

(4) For $R \in \mathcal{A}, N_{S}(R) \cap N_{S}(D)=D T_{R}$ for some complement $T_{R}$ to $D U$ in $N_{S}(D)$ fixing $\omega$.

(5) Let $\mathcal{A}(H)$ be the set of $H$-invariant affine structures on $\Omega$. If $\mathcal{A}(H) \neq \varnothing$, then $|\mathcal{A}(H)|=\left|C_{U D / D}(H)\right|$ and $N_{S}(H)$ is transitive on $\mathcal{A}(H)$.

Proof. First (see [AS, 1.1]), $A=\operatorname{Aut}(D)=D U T$, where $U T$ and its action on $D$ are described in (2). The representation of $A$ on $\Omega^{\prime}=A / D_{\omega} U T$ via right multiplication embeds $A$ in $\operatorname{Sym}\left(\Omega^{\prime}\right)$ in such a way that $D_{\omega} U T$ is the stabilizer of $\omega^{\prime}=D_{\omega} U T \in \Omega^{\prime}$, and so that $A$ preserves the product structure $\mathcal{F}(\mathcal{L})$ on $\Omega^{\prime}$ when $k>1$. Then identifying $\Omega$ with $\Omega^{\prime}$, we conclude that (2) holds, and $N_{S}(D)=A$ with $A \leq N_{S}(\mathcal{F}(\mathcal{L}))$ when $k>1$. By definition of $\mathcal{F}(H)$ in Notation 2.6, $\mathcal{F}=\mathcal{F}(L)=\mathcal{F}(H)$ when $k>1$, completing the proof of (1).

When $k>1$, let $M=N_{S}(\mathcal{F})$ and $Y=F^{*}(M)$. Then using Lemma 1.8, $Y=$ $Y_{1} \times \cdots \times Y_{k}$, with $Y_{i}$ acting faithfully as the alternating group on the $i$ th set $\Omega_{i}$ of partitions in $\mathcal{F}$, and with $Y_{i}$ trivial on $\Omega_{j}$ for $j \neq i$. When $k=1$, let $Y=Y_{1}=F^{*}(S)$, $M=S$, and $\Omega=\Omega_{1}$. Observe that $U T$ is a complement to $Y$ in $M$ with $\left\langle u_{i}\right\rangle Y_{i} \cong S_{8}$ and $\left[u_{i}, Y_{j}\right]=1$ for $i \neq j$.

In $Y_{i}$ there exists $X_{i} \cong E_{8}$ regular on $\Omega_{i}$ with $L_{i}$ acting as $G L\left(X_{i}\right)$ on $X_{i}$. Then $X=X_{1} \times \cdots X_{k} \cong E_{2^{3 k}}$ is regular on $\Omega$, so $D$ stabilizes the affine structure $R=R(X)$ of Lemma 1.4. That is, $R \in \mathcal{A}$. Let $\tilde{M}=N_{S}(R)$, so that $\tilde{M}=\tilde{M}_{\omega} X$ with $\tilde{M}_{\omega}$ acting faithfully as $G L(X)$ on $X$ by Lemma 1.3 .

Observe that $N_{\tilde{M}}(D)=D \tilde{T}$, where $\tilde{T}$ acts faithfully as $\operatorname{Sym}(\mathcal{L})$ on $\mathcal{L}$ and fixes $\omega$. Thus (4) holds.

The representation of $D$ on $X$ is determined up to quasiequivalence, so if $g \in S$ with $D^{g} \leq \tilde{M}$, then there exists $c \in \tilde{M}$ with $L_{i}^{g c} X=L_{i} X$ for each $i$. As $H^{1}\left(L_{i}, X\right) \cong$ $\mathbf{Z}_{2}$, there are two conjugacy classes $L_{i}^{X_{i}}$ and $K_{i}^{X_{i}}$ of complements to $X_{i}$ in $X_{i} L_{i}$. Furthermore, we may choose $K_{i} \leq \tilde{M}_{\omega}$. Therefore, as $L_{i}$ has no fixed points on $\Omega$, $L_{i}^{g c} \in L_{i}^{X_{i}}$, so $D^{g} \in D^{\tilde{M}}$. Thus $\tilde{M}$ is transitive on $D^{S} \cap \tilde{M}$, so $N_{S}(D)$ is transitive on $\mathcal{A}$. Then as $D \tilde{T}=N_{\tilde{M}}(D)$, (3) follows.

Let $B=N_{S}(D)$ and $\bar{B}=B / D$. By (3), $\bar{U}$ is regular on $\mathcal{A}$. Assume that $\mathcal{A}(H) \neq \varnothing$; then conjugating in $U$, we may assume that $H \leq \tilde{M}$. Then as $\bar{U}$ is regular on $\mathcal{A}$, it follows that $C_{\bar{U}}(\bar{H})$ is regular on $\mathcal{A}(H)$, so as $C_{\bar{U}}(\bar{H})=\overline{N_{U D}(H)}, N_{U D}(H)$ is transitive on $\mathcal{A}(H)$, establishing (5). 
We next prove Proposition 5. Assume the hypothesis of Proposition 5. By Lemma 4.5, $M$ is not complemented, while by Lemma 4.8, $M$ is not doubled or diagonal. Therefore $M$ is affine or semisimple. Set $X=F^{*}(M)$.

Suppose that $M$ is affine. Then by Lemma 4.3, $H$ is octal semisimple. By Lemma 7.1(5), $N_{S}(H)$ is transitive on $\mathcal{A}(H)$. Thus conclusion (2) of Proposition 5 holds in this case, so we may assume that $M$ is semisimple, and adopt Notation 5.2.

If $H$ is product decomposable, then conclusion (3) of Proposition 5 holds by Lemma 5.11(6). Hence we may assume that $H$ is product indecomposable. Then by Lemma $5.12(1), b=b(M, H)=1$, so each component of $H$ is contained in a component of $M$. By Lemma 5.3(4), $H$ is transitive on the components of $M$. Finally, by Lemma 5.12(2), the map $K \mapsto \mathcal{F}(H, K)$ is a bijection of $\mathcal{O}_{H}\left(N_{H}(L)\right)^{\prime}$ with $\mathcal{F}(H)$. This completes the proof of Proposition 5.

We now prove Proposition 6. Assume the hypothesis of that proposition. By Proposition 4, $H$ is not affine. Thus Hypothesis 6.1 is satisfied, so we can adopt Notation 6.2. By Lemma 4.8, $H$ is not semisimple. If $H$ is doubled then conclusion (2) of Proposition 6 holds by Lemma 6.5, so we may assume that $H$ is not doubled. Similarly, if $H$ is diagonal then conclusion (1) of Proposition 6 holds by Lemma 6.6, so we may assume that $H$ is complemented. Then Lemma 6.7 says that conclusion (3) of Proposition 6 holds, completing the proof of the proposition.

Finally, we prove Proposition 7, so we assume the hypothesis of Proposition 7. By Proposition 3, $M$ is not affine. By Lemma 4.5, $M$ is not complemented, and by Lemma 4.8, $M$ is not doubled. If $M$ is diagonal then conclusion (1) of Proposition 7 holds by Proposition 6. Thus we may assume that $M$ is semisimple, and adopt Notation 5.2. By Lemma 5.6, $b=b(M, H)=1$, so each component of $H$ is contained in a component of $M$. By Lemma 5.3(4), $H$ is transitive on the components of $M$. Finally, by Lemma 5.5(8) and Lemma 5.6(2), the map $K \mapsto \mathcal{F}(H, K)$ is a bijection of $\mathcal{O}_{H}\left(N_{H}(\sigma)\right)^{\prime}$ with $\mathcal{F}(H)$. This completes the proof of Proposition 7.

\section{The proofs of Propositions 8, 9, and 11}

In this section we assume that Hypothesis 2.1 is satisfied.

Lemma 8.1. Assume that $M \in \mathcal{O}_{G}(H), H$ is complemented, and $M$ is doubled. Then $F^{*}(M)=D C_{G}(D)$.

Proof. Let $X=F^{*}(M)$. Replacing $M$ by $H X$, we may assume that $M=H X$, so that also $M=H_{\omega} X$. As $M$ is doubled, $M$ has two minimal normal subgroups $X_{1}$ and $X_{2}, X=X_{1} \times X_{2}$, and $X_{\omega}$ is a full diagonal subgroup of $X$ with respect to this direct sum decomposition. Let $I=\{1, \ldots, k\}$ and $\mathcal{L}=\left\{L_{i} \mid i \in I\right\}$ the set of components of $X_{1}$. Let $\pi_{i}: X \rightarrow X_{i}$ be the projection map, $\sigma_{i}=\pi_{i \mid X_{\omega}} \rightarrow X_{i}$ the restriction of $\pi_{i}$ to $X_{\omega}$, and $\alpha=\sigma_{1}^{-1} \sigma_{2}: X_{1} \rightarrow X_{2}$. Then $\alpha$ is an $H_{\omega}$-equivariant isomorphism.

By Lemma $4.3, D \leq X$, and as $H$ is complemented, $D$ is regular on $\Omega$ and the unique minimal normal subgroup of $H$. Thus if $D \cap X_{i} \neq 1$ for some $i$, then $D \leq X_{i}$, and then as $|D|=|\Omega|=\left|X_{i}\right|$, we have $D=X_{i}$. Then the lemma follows 
from Lemma 4.6 in this case, so we may assume that $D \cap X_{i}=1$ for $i=1,2$. Then as $|D|=\left|X_{i}\right|, D$ is also a full diagonal subgroup of $X$, so writing $\rho_{i}: D \rightarrow X_{i}$ for the projection of $D$ on $X_{i}$, as above, we obtain an $H_{\omega}$-equivariant isomorphism $\beta=\rho_{1}^{-1} \rho_{2}: X_{1} \rightarrow X_{2}$. Let $D_{i}=L_{i} \rho_{1}^{-1}$. As $\rho_{1}: D \rightarrow X_{1}$ is an isomorphism, $\mathcal{D}=$ $\left\{D_{i} \mid i \in I\right\}$ is the set of components of $H$.

We now argue as in the last few paragraphs of the proof of Lemma 6.7 to obtain a contradiction. As in Sections 5 and 6, we represent $M$ on $I$ so that the map $i \mapsto L_{i}$ is an equivalence of that representation with the representation of $M$ on $\mathcal{L}$ via conjugation. As $\rho_{i}$ is an $H$-equivariant isomorphism and $H_{\omega}$ is transitive on $\mathcal{D}, H_{\omega}$ is also transitive on $\mathcal{L}$, and hence also on $I$.

Let $\gamma=\alpha \beta^{-1}: X_{1} \rightarrow X_{1}$. Then $\gamma \in \operatorname{Aut}\left(X_{1}\right)$ is an $H_{\omega}$-equivariant automorphism of $X_{1}$, which induces $\tau \in \operatorname{Sym}(I)$ commuting with the action of $H_{\omega}$ on $I$. As $H_{\omega}$ is transitive on $I$, the set $\Sigma$ of orbits of $T=\langle\tau\rangle$ on $I$ is an $H_{\omega}$-invariant regular partition of $I$. Let $J$ be an orbit of $T$ on $I, X_{J}=\left\langle L_{j} L_{j} \alpha: j \in J\right\rangle$, $D_{J}=\left\langle D_{j}: j \in J\right\rangle, H_{J}=N_{H_{\omega}}(J) X_{J}$, and $\Omega_{J}=\omega X_{J}$. For $j \in J, D_{j} \leq L_{j} L_{j} \beta$ and $L_{j} \beta=L_{j} \gamma^{-1} \alpha=L_{j \tau^{-1}} \alpha \leq X_{J}$, so $D_{J} \leq X_{J}$. Arguing as in the proof of Lemma 6.7, $D_{J}$ is a minimal normal subgroup of $H_{J}$ regular on $\Omega_{J}$, so $H_{J}$ acts primitively on $\Omega_{J}$ as a complemented or doubled group. Then $M_{J}=X_{J} H_{J}$ acts primitively on $\Omega_{J}$ as a doubled group with minimal normal subgroups $X_{J, i}=X_{i} \cap X_{J}, i=1,2$. If $H_{J}$ is doubled, then by Lemma 4.8(3), $D_{J}=X_{J, i}$ for $i=1$ or 2, contradicting $D \cap X_{i}=1$. Thus $H_{J}$ is complemented, so proceeding by induction on $|\Omega|$, we may assume that $\Omega_{J}=\Omega$. Thus $T$ is transitive on $I$, so as $\tau \in C_{\operatorname{Sym}(I)}\left(H_{\omega}\right), H_{\omega}$ acts as $T$ on $I$. Therefore $\operatorname{Aut}_{H_{\omega}^{\infty}}(D)$ is contained in the kernel of the action of $\operatorname{Aut}(D)$ on $\mathcal{D}$, so $H_{\omega}^{\infty} \leq D_{\omega}=1$. Thus $H_{\omega}$ is solvable, whereas $H$ is complemented, so $\operatorname{Inn}\left(D_{1}\right) \leq \operatorname{Aut}_{H_{\omega}}\left(D_{1}\right)$, giving a contradiction.

We now give proofs of Propositions 8, 9, and 11.

Assume the hypothesis of Proposition 8. By Propositions 4, 5, and 7, $H$ is not affine, semisimple, or diagonal, so $H$ is doubled or complemented. In the first case conclusion (1) of Proposition 8 holds by Lemma 4.8(3), while in the second case conclusion (2) of Proposition 8 holds by Lemma 8.1. This establishes Proposition 8.

Assume the hypothesis of Proposition 9. By Proposition 3, $M$ is not affine, and by Lemma 4.5, $M$ is not complemented. If $M$ is diagonal or doubled, then the proposition follows from Propositions 6 or 8, respectively. Therefore we may assume that $M$ is semisimple, and adopt Notation 5.2. By Lemma 5.6(1), each component $L$ of $H$ is contained in a component of $M$, while by Lemma 5.3(4), $H$ is transitive on the components of $M$. Then conclusion (2) of Proposition 9 holds by Lemma 5.6(2). This completes the proof of Proposition 9.

Assume the hypothesis of Proposition 11. Thus $H$ is complemented and $M \in \mathcal{O}_{G}(H)$. By Proposition 3, $M$ is not affine. If $M$ is diagonal, doubled, or complemented, then conclusion (3), (4), or (1) of Proposition 11 holds by Proposition 6, 8, or 10, respectively. Thus we may assume that $M$ is semisimple, and adopt the notation of Section 5. It remains to show that the map $\phi: K \mapsto \mathcal{F}(H, K)$ 
(see Notation 2.6) is a bijection of $\mathcal{O}_{H}\left(N_{H}(L)\right)^{\prime}$ with $\mathcal{F}(H)$, where $L$ is some choice of component of $H$, that $L$ is contained in a component of $M$, and that $H$ is transitive on the components of $M$. By Lemma 5.3(4), $H$ is transitive on the components of $M$. Recall from Lemma 5.4(3) that $L$ is contained in a component of $M$ if and only if the parameter $b=b(M, H)$ of Notation 5.2 is 1 . From Notation $2.6, \mathcal{F}(H, K) \in \mathcal{F}(H)$ and $\phi$ is injective, so to show that $\phi$ is a bijection we must show that $\phi$ is surjective. That is, we must show that $M=\mathcal{F}(H, K)$ for some $K \in \mathcal{O}_{H}\left(N_{H}(L)\right)^{\prime}$. But if $b=1$ then $\mathcal{F}(M)=\mathcal{F}\left(H, H_{1}\right)$ by Lemma 5.5(6), where $X_{1}$ is the component of $X$ containing $L$ and $H_{1}=N_{H}\left(X_{1}\right)$. Thus it remains to show that $b=1$.

If $H$ is maximal in $M$ then $b=1$ by Lemma 5.13. Thus we may assume that $H \leq M^{\prime}<M$ with $H$ maximal in $M^{\prime}$. Let $X^{\prime}=F^{*}\left(M^{\prime}\right)$. By induction on $n=$ $|M: H|$, the pair $\left(M^{\prime}, H\right)$ satisfies one of the conclusions of Proposition 11, so, in particular, $L$ is contained in a component $L^{\prime}$ of $X^{\prime}$. Thus if $L^{\prime}$ is contained in a component of $M$, then $b=1$ and the proof is complete. If $M^{\prime}$ is complemented, then as $\left|M: M^{\prime}\right|<|M: H|, L^{\prime}$ is contained in a component of $M$ by induction on $n$. If $M^{\prime}$ is diagonal or doubled, then $b\left(M, M^{\prime}\right)=1$ by Lemma 5.6(1), so $L^{\prime}$ is contained in a component of $M$. Thus we may assume that $M^{\prime}$ is semisimple, and that $b\left(M, M^{\prime}\right) \neq 1$. Therefore by parts (3) and (4) of Lemma 5.8, $M^{\prime}$ is octal. But as $L \leq L^{\prime}, b\left(M^{\prime}, H\right)=1$, so applying Lemma 5.5(5) to the pair $\left(M^{\prime}, H\right)$, we conclude that $L^{\prime}$ is the alternating group on $\omega L^{\prime}$, in contradiction to $M^{\prime}$ being octal. Thus the proof of Proposition 11 is at last complete.

See Notation 2.6 and Example 1.6 for the definition of $\mathcal{D}(H)$ and $\mathcal{F}(\mathcal{D})$ appearing in the next theorem.

THEOREM 8.2. Assume that $M_{1}$ and $M_{2}$ are distinct subgroups of $G$ maximal subject to $F^{*}(G) \not M_{i}$, such that $H=M_{1} \cap M_{2}$ is primitive, and $M_{1}$ is not semisimple.

(1) $|\Omega|=p^{f k}$ for some prime $p$ and integers $f, k$ such that $p^{f} \geq 5$ and $k \geq 1$.

(2) $M_{1}$ is affine.

(3) One of the following statements holds:

(i) $|\Omega|=p, H$ is affine with $F^{*}(H)=F^{*}\left(M_{1}\right), M_{2}$ is almost simple, and $H=N_{M_{2}}(D)$;

(ii) $k>1, \quad H$ is affine with $F^{*}(H)=F^{*}\left(M_{1}\right), M_{2}$ is semisimple, and there exists $\mathcal{D}=\left(D_{1}, \ldots, D_{k}\right) \in \mathcal{D}(H)$ such that $M_{2}=N_{G}(\mathcal{F}(\mathcal{D}))$ and $H=N_{M_{1}}(\mathcal{D})$;

(iii) $H$ is octal semisimple with $k$ components, $p^{f}=8, H$ is the wreath product of $L_{3}(2)$ by $S_{k}$, and $M_{1}$ and $M_{2}$ are the stabilizers of the two $H$-invariant affine structures on $\Omega$.

PROOF. Let $Y=F^{*}\left(M_{1}\right)$. As $M_{1}$ is not semisimple, $M_{1}$ is affine or strongly diagonal by Lemma 2.5. Assume the latter and let $k$ be the number of components of $M_{1}$. As $H$ is primitive and $\left|\Sigma\left(M_{1}\right)\right|=1$, Proposition 6 implies that one of the following statements holds: 
(i) $\quad H$ is strongly diagonal and $Y=D$;

(ii) $H$ is doubled, $k=2$, and $Y=D$;

(iii) $\quad H$ is complemented, $k=2$, and $D$ is a component of $Y$.

Conclusion (iii) is impossible since if $H$ is complemented then $H$ is not almost simple. Thus (i) or (ii) holds, and in particular $Y=D$. In case (i), as $M_{2} \in \mathcal{M}(H)$ and $|\Sigma(H)|=1$, Proposition 7 says that $M_{2}$ is strongly diagonal with $F^{*}\left(M_{2}\right)=D$. But then $M_{1}=N_{G}(D)=M_{2}$, contradicting $M_{1} \neq M_{2}$. Therefore case (ii) holds. Hence Proposition 9 says that $M_{2}$ is doubled or diagonal, and $D=F^{*}\left(M_{2}\right)$, or $M_{2}$ is semisimple. But in the first two cases, again $M_{1}=N_{G}(D)=M_{2}$, for the same contradiction. Thus $M_{2}$ is semisimple, so by Proposition 9, a component $D_{1}$ of $H$ is contained in a component of $M_{2}$, and $H$ is transitive on the components of $M_{2}$. Then as $D_{1} \unlhd H, M_{2}$ is almost simple, contrary to Proposition 1 .

Therefore $M_{1}$ is affine, so conclusions (1) and (2) of Theorem 8.2 hold, except possibly $p^{k}<5$. As $H$ is primitive, Lemma 4.3 says that either

(a) $D \leq Y$; or

(b) $H$ is octal semisimple, $D=L_{1} \times \cdots \times L_{k}, L_{i} \cong L_{3}(2), p^{f}=8, H Y$ is affine, $\mathcal{D}=\left\{Y_{1}, \ldots, Y_{k}\right\} \in \mathcal{D}(H Y)$, and $\mathcal{F}(H)=\mathcal{F}(\mathcal{D})$, where $Y_{i}=\left[Y, L_{i}\right] \cong E_{8}$.

Suppose that case (a) holds. Then $D$ is solvable, so $H$ is affine and $D=Y$. By Proposition 4, one of the following statements holds:

(I) $\quad M_{2}$ is affine and $D=F^{*}\left(M_{2}\right)$;

(II) $H$ is imprimitive on $Y$, and there exists $\mathcal{D}=\left\{D_{1}, \ldots, D_{k}\right\} \in \mathcal{D}(H)$ with $M_{2}=$ $N_{G}(\mathcal{F}(\mathcal{D}))$

(III) $|\Omega|$ is prime and $M_{2}$ is almost simple.

In case (I) we have our usual contradiction, while in cases (II) and (III) conclusions (3ii) and (3i) of Theorem 8.2 hold, respectively.

Finally, assume that case (b) holds. As $M_{1} \neq M_{2}$, Proposition 5 says that either

(A) $\quad M_{2}$ is affine and the stabilizer of an affine structure on $\Omega$ preserved by $H$; or

(B) $\quad M_{2}$ is the stabilizer of $\mathcal{F}(H, K)$ for some $K \in \mathcal{O}_{H}\left(N_{H}\left(L_{1}\right)\right)^{\prime}$.

Suppose that case (A) holds. By Lemma 7.1(4), $N_{M_{1}}(D)=D T_{1}$, where $T_{1}$ acts faithfully as $\operatorname{Sym}(\mathcal{L})$ on $\mathcal{L}=\left\{L_{1}, \ldots, L_{k}\right\}$. Thus $H=D T_{H}$, where $T_{H} \leq T_{1}$ is transitive on $\mathcal{L}$. By Lemma 7.1(2), $U$ is the permutation module for $T_{1}$ and $T_{H}$, so by Lemma 7.1(5), $|\mathcal{A}(H)|=\left|C_{U D / D}(H)\right|=2$. Thus $M_{2}=M_{1}^{u}$, where $u \in N_{U}(H)$ Similarly $\left|C_{U D / D}\left(D T_{1}\right)\right|=2$, so as $T_{H} \leq T_{1}, C_{U D / D}(H)=C_{U D / D}\left(D T_{1}\right)$, and hence $D T_{1} \leq M_{1} \cap M_{2}=H$, so $T_{H}=T_{1} \cong S_{k}$. But now conclusion (3iii) of Proposition 12 holds.

So assume that case (B) holds, let $Y_{1, K}=\left\langle Y_{1}^{K}\right\rangle$, and $\mathcal{D}_{K}=Y_{1, K}^{H}$. Then $\mathcal{D}_{K} \in$ $\mathcal{D}(H Y)$ and, by Notation 2.6, $\omega D_{\gamma_{K}^{h}}=\omega Y_{1, K}^{h}$, so by Lemma 1.7, $\mathcal{F}\left(\mathcal{D}_{K}\right)=\mathcal{F}(H, K)$. Thus $H Y \leq M_{1} \cap M_{2}=H$, giving a contradiction. This completes the proof of the theorem.

See Lemma 5.11(6) for the definition of $\mathcal{F}^{2}(H)$ appearing in the next lemma. 
LeMma 8.3. Assume that $H$ is almost simple and product decomposable.

(1) If $M \in \mathcal{O}_{G}(H)^{\prime}$ with $M$ almost simple, then $F^{*}(M)=F^{*}(H)$.

(2) $N_{G}\left(\mathcal{F}^{2}(H)\right)$ is the unique maximal member of $\mathcal{O}_{G}(H)^{\prime}$.

Proof. Let $d^{2}=\left|D: D_{\omega}\right|=|\Omega|$, and $A_{i} \in \mathcal{O}_{D}\left(D_{\omega}\right), i=1,2$, with $\left|A_{i}: D_{\omega}\right|=$ $\left|D: A_{i}\right|=d$ as in Definition 5.10. Set $\Lambda=\operatorname{Aut}(D)$ and $\Sigma=N_{\Lambda}\left(D_{\omega}\right)$. From Lemma 3.4, $\Lambda=\operatorname{Inn}(D) \Sigma,\left|\Sigma: N_{\Sigma}\left(A_{1}\right)\right|=2$ with $\Sigma=H_{\omega} N_{\Sigma}\left(A_{1}\right)$, and $A_{1}^{h}=A_{2}$ for $h \in H_{\omega}-N_{\Sigma}\left(A_{1}\right)$. It follows that $N_{S}(D) \cong \Lambda$ and from Lemma 5.11(6), $N_{S}(D) \leq$ $N_{S}\left(\mathcal{F}^{2}(H)\right)$. Thus if (1) holds then each almost simple member of $\mathcal{O}_{G}(H)^{\prime}$ is contained in $\tilde{M}=N_{G}\left(\mathcal{F}^{2}(H)\right)$. On the other hand, by Proposition 2, each member of $\mathcal{O}_{G}(H)^{\prime}$ which is not almost simple is contained in $\tilde{M}$. Thus (1) implies (2), so it remains to prove (1). Therefore we may assume that $M \in \mathcal{O}_{G}(H)^{\prime}$ with $M$ almost simple and $F^{*}(M) \neq D$, and it remains to derive a contradiction. Choose $H, M$ so that $H$ is maximal subject to these constraints, and $M$ is minimal subject to this further constraint. From the maximality of $H, H=N_{M}(D)$.

Let $H \leq M^{\prime} \leq M$ with $H$ maximal in $M^{\prime}$. By Proposition 2, $M^{\prime}$ is semisimple, and hence, as $M^{\prime} \leq M, M^{\prime}$ is almost simple by Lemma 4.4. Therefore as $H=N_{M}(D)$, $M=M^{\prime}$ by minimality of $M$. That is, $H$ is maximal in $M$.

By Lemma 2.4, $M$ is primitive on $\Omega$, so we have shown that:

(a) $H$ is a maximal subgroup of the primitive almost simple subgroup $M$ of $S$ on $\Omega$;

(b) $H$ is almost simple, primitive, and product indecomposable on $\Omega$ of order $d^{2}$.

To complete the proof we inspect the list of possible inclusions in part (B) of the main theorem of [LPS1] for pairs $(H, M)$ satisfying (a) and (b). In particular, by (b) and Definition $5.10, F^{*}(H) \cong A_{6}, M_{12}$, or $S p_{4}(q), q>2$ even, and $n=|\Omega|=d^{2}$, where $d=6,12$, or $q^{2}\left(q^{2}-1\right) / 2$, respectively. As there are no such pairs, we obtain a contradiction, which completes the proof.

THEOREM 8.4. Assume that $M_{1}$ and $M_{2}$ are distinct subgroups of $G$ maximal subject to $F^{*}(G) \not M_{i}$, such that $H=M_{1} \cap M_{2}$ is primitive, $M_{1}$ is almost simple, and $M_{2}$ is semisimple. Then the following statements hold:

(1) $M_{2}$ is also almost simple.

(2) Either $H$ is almost simple, or $|\Omega|$ is prime and $H$ is affine.

PROOF. If $|\Omega|$ is prime, then as $M_{2}$ is semisimple, we conclude from Lemma 2.2 that (1) holds, and then (2) follows from Lemma 4.4.

Thus we may assume that $|\Omega|$ is not prime. Therefore $H$ is almost simple by Lemma 4.4, so (2) holds. Next by Proposition 2, either (1) holds or $|\Omega|=8$ and $H \cong L_{3}(2)$ is octal. But now $\mathcal{M}(H)$ consists of the stabilizers of the two affine structures on $\Omega$ if $G=F^{*}(S)$, or that pair of subgroups together with $N_{S}(H) \cong$ $P G L_{2}(7)$ if $G=S$. But in either case the hypothesis of the theorem is not satisfied. Thus the proof of Theorem 8.4 is complete.

LEMMA 8.5. Assume that $H$ is almost simple, product indecomposable, and not octal. Then each member of $\mathcal{O}_{S}(H)$ is almost simple, product indecomposable, and not octal. 
Proof. Let $M \in \mathcal{O}_{S}(H)^{\prime}$. By Proposition 2, $M$ is almost simple, so it remains to show that $M$ is product indecomposable and not octal. Proceeding by induction on $|M: H|$, we may assume that $M$ is octal or product decomposable, and that $H$ is maximal in $M$. If $M$ is octal then $|\Omega|=8$ and $M \cong L_{3}(2)$, so all proper subgroups of $M$ are solvable, contradicting $H<M$. Therefore $M$ is product decomposable. Hence by Definition $5.10,|\Omega|=d^{2}$, where $d$ is 6,12 , or $q^{2}\left(q^{2}-1\right) / 2$, and $M$ appears in case (i), (ii), or (iii) of Definition 5.10, respectively. As $M$ is product decomposable but $H$ is not, $F^{*}(M) \neq D$. Thus the pair $D, F^{*}(M)$ appears in one of [LPS1, Tables III-VI]. Inspecting these tables for examples with $|\Omega|=d^{2}$ and $F^{*}(M)$ listed in Definition 5.10, we obtain a contradiction.

\section{The proofs of Theorems 12 and 13}

In this section we assume Hypothesis 2.1.

We begin with a proof of Theorem 12, so assume the hypothesis of that theorem. Suppose first that $M_{1}$ is not semisimple. Then by Theorem 8.2, $M_{1}$ is affine and conclusion (ii) or (iii) of Theorem 8.2(3) holds. But now conclusion (3) or (4) of Theorem 12 holds. Therefore we may assume that $M_{1}$ and $M_{2}$ are semisimple.

Suppose next that $M_{1}$ is almost simple. Then by Theorem 8.4, $M_{2}$ and $H$ are almost simple, so conclusion (1) of Theorem 12 holds. Hence we may assume that neither $M_{1}$ nor $M_{2}$ is almost simple. Then by maximality of $M_{i}$ and Lemma 2.5, $M_{i}=N_{G}\left(\mathcal{F}\left(M_{i}\right)\right)$, so conclusion (2) of Theorem 12 holds, completing the proof of Theorem 12.

The remainder of the section is devoted to a proof of Theorem 13. In particular, we assume the hypothesis and notation of Theorem 13. For example, $\mathcal{M}$ denotes the set of maximal overgroups of $H$ in $G$. In addition, assume that $H$ is a counterexample to Theorem 13. Set $L=F^{*}(G)$, so that $L$ is the alternating group on $\Omega$. We begin a short series of reductions.

LEMMA 9.1. Suppose that $G=S$ and $H \leq L$.

(1) L and $N_{G}(H)$ are in $\mathcal{M}$.

(2) For each $M \in \mathcal{M}-\left\{N_{G}(H)\right\}, M \cap N_{G}(H)=H$.

(3) $H$ and $N_{G}(H)$ are almost simple.

Proof. Parts (1) and (2) follow from Lemma 3.7. If $N_{G}(H)$ is the stabilizer in $S$ of an affine structure, regular product structure, or diagonal structure, then by (1) and (2), $H$ is the stabilizer in $L$ of that structure, and hence is maximal in $L$ by Remark 2.7. But then by Lemma 3.8, case (6) of Theorem 13 holds, contrary to the choice of $H$ as a counterexample. Hence $N_{G}(H)$ is almost simple by Lemma 2.5, so $H$ is also almost simple, establishing (3).

LEMMA 9.2.

(1) $H$ is not almost simple.

(2) If $G=S$ then $H \nless L L$.

(3) $\mathcal{M}=\mathcal{M}(H)$. 
Proof. Suppose first that $H$ is almost simple. As $H$ is a counterexample to Theorem 13, case (2) of that theorem does not hold, so either $H$ is octal or product decomposable, or some member of $\mathcal{O}_{G}(H)$ is not almost simple, product indecomposable, and not octal. The latter contradicts Lemma 8.5, so the former holds. Hence by Proposition 2, one of the following statements holds:

(i) $H \cong L_{3}(2)$ is octal, and either

(a) $G=L, \mathcal{M}=\left\{M_{1}, M_{2}\right\}$ with $M_{1}$ and $M_{2}$ affine, and $\mathcal{O}_{G}(H)=$ $\left\{H, M_{1}, M_{2}, G\right\}$; or

(b) $G=S, \mathcal{M}=\left\{L, N_{S}(H)\right\}$, and $\mathcal{O}_{G}(S)=\left\{H, M_{1}, M_{2}, L, N_{G}(H), G\right\}$.

(ii) $H$ is product decomposable.

Conclusion (i) contradicts the choice of $H$ as a counterexample. If conclusion (ii) holds, then by Lemma 8.3, $\mathcal{M}(H)=\left\{N_{G}\left(\mathcal{F}^{2}(H)\right)\right\}$. Therefore by hypothesis $(0.1)$ of Theorem 13, $\mathcal{M} \neq \mathcal{M}(H)$, so $G=S$ and $H \leq L$. Therefore $H=N_{L}\left(\mathcal{F}^{2}(H)\right)$ by Lemma 9.1, which is not the case. Hence (1) is established.

Observe that (1) and Lemma 9.1(3) imply (2), while (2) implies (3).

LEMMA 9.3. No member of $\mathcal{M}$ is almost simple.

Proof. Assume that $M \in \mathcal{M}$ is almost simple. Then by Lemma 9.2(3) and Theorem $12, H$ is almost simple, contrary to Lemma 9.2(1).

LEMMA 9.4. There exists $M \in \mathcal{M}$ such that $M$ is not semisimple.

Proof. Assume otherwise and let $M \in \mathcal{M}$. By Lemma 9.3, $M$ is not almost simple, so by Lemma 2.5 and maximality of $M, M=N_{G}(\mathcal{F}(M))$. As $H \leq M, \mathcal{F}(M) \in$ $\mathcal{F}(H)$, so conclusion (1) of Theorem 13 holds, contrary to the choice of $H$ as a counterexample.

Let $\Delta$ be the set of pairs $\left(M_{1}, M_{2}\right)$ of distinct $M_{i} \in \mathcal{M}, i=1,2$, such that $H=M_{1} \cap M_{2}$ and $M_{1}$ is not semisimple. By Lemma 9.4 and hypothesis (0.1) of Theorem 13, $\Delta \neq \varnothing$.

Lemma 9.5. Let $\left(M_{1}, M_{2}\right) \in \Delta$. Then either

(1) $\quad H$ is affine, $M_{1}=N_{G}(D)$, and $M_{2}=N_{G}(\mathcal{D})$ for some $\mathcal{D} \in \mathcal{D}(H)$; or

(2) $H \cong L_{3}(2) \mathrm{wr} \mathrm{S}_{\mathrm{k}}$ is octal semisimple, and $M_{1}$ and $M_{2}$ are the stabilizers of the two $H$-invariant affine structures on $\Omega$.

Proof. As $\left(M_{1}, M_{2}\right) \in \Delta, H, M_{1}, M_{2}$ satisfy one of the conclusions of Theorem 12 . Then as $M_{1}$ is not semisimple, the lemma follows by inspection of the possibilities listed in Theorem 12.

Suppose that $\left(M_{1}, M_{2}\right) \in \Delta$ satisfies Lemma 9.5(2). Then $M_{i}$ is perfect, so $M_{i} \leq L$. Thus $G=L$ by maximality of $M_{i}$. By Proposition 5, and as $H$ is primitive on the set of components of $H, \mathcal{M}(H)=\left\{M_{1}, M_{2}, M_{3}\right\}$, where $M_{3}=N_{G}(\mathcal{F}(H))$. By hypothesis (0.1) in Theorem $13, M_{i} \cap M_{3}=H$ for $i=1$ or 2, contrary to Theorem 12 . 
We have shown that for each $\left(M_{1}, M_{2}\right) \in \Delta,\left(M_{1}, M_{2}\right)$ satisfies Lemma 9.5(1). In particular, $H$ is affine and $M_{1}=N_{G}(D)$, so all members of $\mathcal{M}-\left\{N_{G}(D)\right\}$ are semisimple. Then it follows from Proposition 4 that case (3) of Theorem 13 holds, contrary to the choice of $H$ as a counterexample to Theorem 13. This contradiction completes the proof of Theorem 13.

\section{References}

[A1] M. Aschbacher, 'Overgroups of Sylow subgroups in sporadic groups', Mem. Amer. Math. Soc. 60(343) (1985), 1-235.

[A2] - Finite Group Theory (Cambridge University Press, Cambridge, 1986).

[A3] - 'On intervals in subgroup lattices of finite groups', J. Amer. Math. Soc. 21 (2008), 809-830.

[A4] - 'Signalizer lattices in finite groups', Michigan Math. J. 58(1) (2009), 79-103.

[AS] M. Aschbacher and L. Scott, 'Maximal subgroups of finite groups', J. Algebra 92 (1985), 44-80.

[BL] R. Baddeley and A. Lucchini, 'On representing finite lattices as intervals in subgroup lattices of finite groups', J. Algebra 196 (1997), 1-100.

[BP] R. Baddeley and C. Praeger, 'On primitive overgroups of quasiprimitive permutation groups', J. Algebra 263 (2003), 294-344.

[BPS1] R. Baddeley, C. Praeger and C. Schneider, 'Transitive simple subgroups of wreath products in product action', J. Aust. Math. Soc. 77 (2004), 55-72.

[BPS2] - 'Innately transitive subgroups of wreath products in product action', Trans. Amer. Math. Soc. 358 (2006), 1619-1641.

[BPS3] — 'Quasiprimitive groups and blow-up decompositions', J. Algebra 311 (2007), 337-351.

[GLS3] D. Gorenstein, R. Lyons and R. Solomon, The Classification of the Finite Simple Groups, Number 3, Mathematical Surveys and Mongraphs, 40 (American Mathematical Society, Providence, RI, 1999).

[Gu] R. Guralnick, 'Subgroups of prime power index in a simple group', J. Algebra 81 (1983), 304-311.

[K] P. Kleidman, 'The maximal subgroups of the 8-dimensional orthogonal groups $P \Omega_{8}^{+}(q)$ and of their automorphism groups', J. Algebra 110 (1987), 173-242.

[LPS1] M. Liebeck, C. Praeger and J. Saxl, 'A classification of the maximal subgroups of the finite alternating and symmetric groups', J. Algebra 111 (1987), 365-383.

[LPS2] _ 'The maximal factorizations of the finite simple groups and their automorphism groups', Mem. Amer. Math. Soc. 86(432) (1990), 1-151.

[NZ] I. Niven and H. Zuckerman, An Introduction to the Theory of Numbers (Wiley, New York, 1980).

[PP] P. Palfy and P. Pudlak, 'Congruence lattices of finite algebras and intervals in subgroup lattices of finite groups', Algebra Universalis 61 (1980), 22-27.

[P] C. Praeger, 'The inclusion problem for finite primitive permutation groups', Proc. London Math. Soc. 60 (1990), 68-88.

[T] J. Tits, 'A local approach to buildings', in: The Geometric Vein. The Coxeter Festschrift (Springer, New York, 1982), pp. 519-547.

\section{MICHAEL ASCHBACHER, California Institute of Technology, Pasadena, California 91125, USA \\ e-mail: asch@its.caltech.edu}

